\title{
A Low Temperature Expansion for Classical $N$-Vector Models. II. Renormalization Group Equations
}

\section{Tadeusz Balaban}

Rutgers University, Department of Mathematics, New Brunswick, NJ 08903, USA

Received: 25 July 1995/Accepted: 30 May 1996

\begin{abstract}
This paper continues the analysis of the low temperature expansions for classical $N$-vector models started in [1]. A main part of it is a derivation of renormalization group equations and a construction of their solutions. To do this we have to introduce "a fluctuation integral" connected with a next renormalization transformation, and to make its preliminary analysis. The results of the paper are summarized in theorems stating that the renormalization transformation preserves the space of densitites, or actions described inductively in [1].
\end{abstract}

\section{Introduction, Formulation of Main Theorems}

This paper is a continuation of the paper [1], and we use the notations, definitions and results established there, as well as in the following papers [2, 3]. Our main purpose here is to define renormalization procedures for new contributions to the effective actions and the generating functionals, and to set up and analyse renormalization group equations for the "running" coefficients in the main term of the effective action. The new contributions come from "small field" fluctuation integrals connected with a successive $k+1^{\text {st }}$ renormalization transformation, and our other purpose is to set up such fluctuation integrals, and to define the new contributions. The renormalization procedures, and in particular the renormalization group equations have to be defined under the assumption that the effective actions are defined on the whole lattice, i.e., in the framework of paper [1]. To give some more precise shape to this paper we assume also that the fluctuation integrals are defined on the whole lattice, i.e., the lattice is "a small field region," but it is very easy to generalize constructions and results obtained in this case to general cases when small field regions are subregions of the whole lattice. If the renormalization procedure and the renormalization group flow depended on such a region, then a new renormalized 
action would depend on it also, and even after a localization expansion terms localized in smaller subdomains would depend on it. This would be inconsistent with one of the fundamental properties of our procedure, that an expression constructed by some operation from previous expressions, and subsequently localized in some domain, depends on the previous expressions localized in the same domain also. To preserve this property we define the renormalization group flow globally on the whole lattice, actually even on the whole lattice $\mathbb{Z}^{d}$, so it is independent also of the torus $T$. This was not stated so clearly in [1], so we stress it again here that the renormalization conditions in [1] can be formulated in terms of functions defined by proper "thermodynamic limits," as we do it in the inductive hypotheses formulated in this paper. With the above assumption we present the constructions and results of this paper as a theorem that the renormalization transformations together with the renormalization procedures preserve the form and bounds of the effective densities described in [1]. This is a main theorem of many renormalization group approaches, and our final results will have this form also, but for complete densities including contributions from "large field domains." Let us stress again that in this paper the theorem gives only a coherent way of presentation of results, we have explained above our main goals. We need here more precise formulations of some inductive hypotheses, so we start writing them in a proper form. Let us stress that the changes in the hypotheses make them more precise and restrictive, so they do not affect the considerations and results of [1]. We only formulate them here, and we refer the reader to Sect. 2 of [1] for all remarks, explanations and definitions.

Let us recall the definition of the function determining the main term in effective actions, which is

$$
\begin{gathered}
A_{k}(\psi, \phi ; h, a, \lambda, v)=\frac{1}{2} a\left\|\psi-Q_{k} \phi\right\|_{1}^{2}+\frac{1}{2}\left\|\partial^{n} \phi\right\|^{2}+\frac{\lambda}{8}\left\|\phi^{2}-1\right\|^{2}+\frac{v}{2}\|\phi-h\|^{2}, \\
\eta=L^{-k}, \frac{1}{2}<a<\frac{3}{2}, \lambda \geqq 1,0<v \leqq 1
\end{gathered}
$$

where a "new" spin configuration $\psi$ is defined on $T_{1}^{(k)}$, an "old" spin configuration $\phi$ is defined on $T_{\eta}$, both have values in $\mathbb{R}^{N}, h$ belongs to a neighborhood of the unit sphere $S^{N-1}$ in $\mathbb{R}^{N}$. The norms in (1.1) are $L^{2}$-norms, the first on the unit lattice $T_{1}^{(k)}$, the remaining three on the lattice $T_{\eta}$. We consider densities $\rho_{k}$ satisfying the following inductive hypotheses.

(H.1) There exist positive coefficients $\beta_{k}, a_{k}, \lambda_{k}, v_{k}, \frac{1}{2}<a_{k}<\frac{3}{2}, \lambda_{k} \geqq L^{2 k}, 0<$ $v_{k} \leqq 1$, functioncs $\mathscr{E}_{k}\left(\psi_{k}, h\right), \mathscr{F}_{k}\left(\psi_{k}, h, g\right)$, and constants $E_{k}, \alpha_{0}, \gamma$ such that

$$
\begin{gathered}
\rho_{k}\left(\psi_{k}\right)=\chi_{k} \exp \left[\mathscr{A}_{k}+\mathscr{F}_{k}\left(\psi_{k}, h, g\right)\right] \\
\mathscr{A}_{k}=-\beta_{k} A_{k}\left(\psi_{k}, \phi_{k} ; h, a_{k}, \lambda_{k}, v_{k}\right)+\mathscr{E}_{k}\left(\psi_{k}, h\right)-E_{k}\left|T_{1}^{(k)}\right|,
\end{gathered}
$$

where $\psi_{k}$ is a "new" spin variable on the lattice $T^{(k)}$, and $\phi_{k}=\phi_{k}\left(\psi_{k} ; h, a_{k}, \lambda_{k}, v_{k}\right)$ is the minimal configuration defined in Sect. 2 of [1], and constructed in [2]. The function $\mathscr{E}_{k}\left(\psi_{k}, h\right)$ is defined and real analytic on the space $\Xi_{k}\left(1, \varepsilon_{k}\right)$, where $\varepsilon_{k}=\alpha_{0, k} \eta^{\alpha}, \quad \alpha_{0, k}=\alpha_{0}\left(1+\sum_{n=k}^{\infty} \frac{1}{n^{2}}\right), \alpha_{0}$ is positive and small, $\alpha=\frac{d-2}{2}-\gamma, \gamma$ is a constant from the interval $0<\gamma<\min \left\{\frac{d-2}{2}, \frac{1}{2}\right\}$. The function $\widetilde{\mathscr{F}}_{k}\left(\psi_{k}, h, g\right)$ is 
defined and real analytic on the space $\Xi_{k}\left(1, \varepsilon_{k}\right) \times\{g: g$ is defined on $T$ and has values in $\left.\mathbb{R}^{N},\|g\|_{\ell^{1}}<1\right\}$.

(H.2) There exist functions $\mathscr{E}_{k}^{(j)}\left(y ; \psi_{j}, h\right)$, where $y \in T^{(j)}$ and $\psi_{j}$ is a spin variable on this lattice, such that

$$
\mathscr{E}_{k}\left(\psi_{k}, h\right)=\sum_{j=1}^{k} \mathscr{E}_{k}^{(j)}\left(\psi_{k}^{(j)}\left(\psi_{k}, h\right), h\right),
$$

where

$$
\mathscr{E}_{k}^{(j)}\left(\psi_{j}, h\right)=\sum_{y \in T^{(J)}} \mathscr{E}_{k}^{(j)}\left(y ; \psi_{j}, h\right)
$$

and where $\psi_{k}^{(j)}\left(\psi_{k}, h\right)$ are the minimal configurations of the variational problems (2.6) in [1], constructed in [2]. The functions $\mathscr{E}_{k}^{(j)}\left(y ; \psi_{j}, h\right)$ are defined and real analytic on the space $\Xi_{j}\left(1, \varepsilon_{j}\right)$, where $\varepsilon_{j}=\alpha_{0, k} \xi^{\alpha}, \xi=L^{-j}$, and the coefficients $a_{j}, \lambda_{j}, v_{j}$ in the definition of this space are connected with $a_{k}, \lambda_{k}, v_{k}$ by the equalities

$$
a_{j}=a_{k} \frac{1-L^{-2 k}}{1-L^{-2 j}}, \quad \lambda_{j}=\lambda_{k}\left(L^{j} \eta\right)^{2}, \quad v_{j}=v_{k}\left(L^{j} \eta\right)^{2}
$$

(H.3) The functions $\mathscr{E}_{k}^{(j)}\left(y ; \psi_{j}, h\right)$ satisfy the symmetry properties

$$
\begin{gathered}
\mathscr{E}_{k}^{(j)}\left(y ; R \psi_{j}, R h\right)=\mathscr{E}_{k}^{(j)}\left(y ; \psi_{j}, h\right), \quad R \in O(N), \\
\mathscr{E}_{k}^{(j)}\left(r y ; r \psi_{j}, h\right)=\mathscr{E}_{k}^{(j)}\left(y ; \psi_{j}, h\right),
\end{gathered}
$$

for all Euclidean transformations $r$ of the lattice $T^{(j)}$ onto itself, where $\left(r \psi_{j}\right)(y)=$ $\psi_{j}\left(r^{-1} y\right)$.

(H.4) There exist functions $\mathscr{E}_{k}^{(j)}\left(y, X ; \psi_{j}, h\right)$ defined for localization domains $X \in$ $\mathscr{D}_{j}=\mathscr{D}_{j}(T), y \in X$, such that

$$
\mathscr{E}_{k}^{(j)}\left(y ; \psi_{j}, h\right)=\sum_{X \in \mathscr{D}_{j}: y \in X} \mathscr{E}_{k}^{(j)}\left(y, X ; \psi_{j}, h\right)
$$

A function $\mathscr{E}_{k}^{(j)}\left(y, X ; \psi_{j}, h\right)$ depends on $\psi_{j}$ restricted to the domain $X$, and it does not depend on the torus $T$ if $X$ does not "wind around it," for example if $X \subset\left\{x: m_{\mu}<x_{\mu}<M_{\mu}, \mu=1,2, \ldots, d\right\}$ and $M_{\mu}-m_{\mu}<2 L_{\mu} \xi$, for the torus in the $\xi$-scale. This function has an analytic extension onto the complex space $\Xi_{j}^{c}\left(\mathbb{B}_{j}(X) ; 1, \varepsilon_{j}\right)$ defined in Sect. 4 of [3], and the extension depends on configurations $\left(\psi+\psi^{\prime}, h+h^{\prime}\right)$ restricted to $X$, i.e. on $\left(\psi_{j}+\psi_{j}^{\prime}, h+h^{\prime}\right)$ restricted to $X$ and it satisfies the bound

$$
\left|\mathscr{E}_{k}^{(j)}\left(y, X ; \psi_{j}+\psi_{j}^{\prime}, h+h^{\prime}\right)\right|<E_{0} \exp \left(-\kappa d_{j}(X)\right),
$$

where $E_{0}, \kappa$ are positive constants, $\kappa$ can be chosen arbitrarily large, depending on the size $M$ of large cubes. It is invariant with respect to orthogonal transformations $R \in O(N)$ applied to the configurations, and covariant with respect to Euclidean 
transformations $r$ of the lattice of large cubes in $T^{(j)}$, i.e., the lattice $T^{(j+m)}$, where $M=L^{m}$ is the size of the cubes.

Let us interrupt for a moment formulating the inductive hypotheses, and let us notice that it is the above one which has been strengthened in comparison with the corresponding hypothesis (H.5) in [1]. The analyticity domain, which is now the space $\Xi_{j}^{c}\left(\mathbb{B}_{j}(X) ; 1, \varepsilon_{j}\right)$ restricted to $X$, contains the domain considered in [1], which was the whole lattice space $\Xi_{j}^{c}\left(1, \varepsilon_{j}\right)$ restricted to $X$, in fact it contains any space $\Xi_{j}^{c}\left(\mathbb{B}_{j} ; 1, \varepsilon_{j}\right)$ with $\mathbb{B}_{j}$ "larger" than $\mathbb{B}_{j}(X)$, i.e. $\mathbb{B}_{j} \succ \mathbb{B}_{j}(X)$. An advantage of using the space $\Xi_{j}^{c}\left(\mathbb{B}_{j}(X) ; 1, \varepsilon_{j}\right)$ is that its definition is localized to a relatively small neighborhood of $X$, which is important in constructions of localization expansions. From the fact that each term in the sum on the right-hand side of (H.4) is analytic on the space $\Xi_{j}^{c}\left(1, \varepsilon_{j}\right)$ it follows that the sum, which is the function $\mathscr{E}_{k}^{(j)}\left(y ; \psi_{j}, h\right)$, is analytic on this space, so its restriction to the real subspace $\Xi_{j}\left(1, \varepsilon_{j}\right)$ is real analytic on it. Thus the analyticity assumption in (H.1) follows from the assumption in (H.4), and in the future we will be concerned only with the last one. The hypotheses (H.3), (H.4) imply existence of an effective potential defined by

$$
\mathscr{V}_{k}^{(j)}(\psi, h)=\lim _{T_{\xi} \rightarrow \xi \mathbb{Z}^{d}} \mathscr{E}_{k}^{(j)}(y ; \psi, h)=\lim _{T_{\xi} \rightarrow \xi \mathbb{Z}^{d}} \frac{1}{\left|T_{\xi}\right|} \mathscr{E}_{k}^{(j)}(\psi, h)
$$

where $\psi, h$ are vectors from $\mathbb{R}^{N}$ such that the constant configuration equal to $\psi$ belongs together with $h$ to the space $\Xi_{j}\left(1, \varepsilon_{j}\right)$. The hypothesis (H.4) implies existence of various other thermodynamic limits, like $\mathscr{E}_{k}^{(j, \infty)}\left(y ; \psi_{j}, h\right)=\lim _{T_{\xi} \rightarrow \xi \mathbb{Z}^{d}} \mathscr{E}_{k}^{(j)}\left(y ; \psi_{j}\right.$, $h$ ), and in particular of the function

$$
\begin{aligned}
& \mathscr{G}_{k}^{(j, \infty)}\left(x-x^{\prime}\right)=\lim _{T_{\xi} \rightarrow \xi \mathbb{Z}^{d}}\left(\frac{\partial^{2}}{\partial \psi_{j}(x) \partial \psi_{j}\left(x^{\prime}\right)} \mathscr{E}_{k}^{(j)}\right)\left(e_{1}, e_{1}\right) \\
& =\sum_{y \in \mathbb{Z}^{d}}\left(\frac{\partial^{2}}{\partial \psi_{j}(x) \partial \psi_{j}\left(x^{\prime}\right)} \mathscr{E}_{k}^{(j, \infty)}\right)\left(y ; e_{1}, e_{1}\right), \quad e_{1}=(1,0, \ldots, 0) .
\end{aligned}
$$

We formulate the next inductive hypothesis, the renormalization conditions in terms of $\mathscr{V}_{k}^{(j)}, \mathscr{G}_{k}^{(j, \infty)}$. We assume that

(H.5)

$$
\begin{aligned}
\mathscr{V}_{k}^{(j)}\left(e_{1}, e_{1}\right) & =0,\left(\frac{\partial}{\partial h} \mathscr{V}_{k}^{(j)}\right)\left(e_{1}, e_{1}\right) \cdot e_{1}=0, \\
\left(\frac{\partial}{\partial \psi} \mathscr{V}_{k}^{(j)}\right)\left(e_{1}, e_{1}\right) \cdot e_{1} & =\sum_{y^{\prime} \in T^{(j)}}\left(\frac{\partial}{\partial \psi_{j}\left(y^{\prime}\right)} \mathscr{E}_{k}^{(j)}\right)\left(y ; e_{1}, e_{1}\right) \cdot e_{1}=0, \\
\sum_{x \in \mathbb{Z}^{d}} \operatorname{tr}\left[\mathscr{G}_{k}^{(j, \infty)}(x) \cdot\left(e_{2} \otimes e_{2}\right)\right]|x|^{2} & =-\left.\Delta_{p} \operatorname{tr}\left[\mathscr{G}_{k}^{(j, \infty)}(p) \cdot\left(e_{2} \otimes e_{2}\right)\right]\right|_{p=0} \\
& =-d\left(\frac{\partial^{2}}{\partial p_{\mu}^{2}} \mathscr{G}_{k ; 2,2}^{(j, \infty)}\right)(0)=0, \quad \mu=1,2, \ldots, d .
\end{aligned}
$$


(H.6) The coefficients $\beta_{k}, a_{k}, \lambda_{k}, v_{k}$ are connected with the fixed coefficients $\beta, a, \lambda, v$ by the equalities

$$
\beta_{k}=\beta L^{k(d-2)} \gamma_{k}, \quad a_{k}=a \frac{1-L^{-2}}{1-L^{-2 k}}, \quad \lambda_{k}=\lambda L^{2 k}, \quad v_{k}=v L^{2 k} \delta_{k},
$$

or $\beta_{k}=\beta L^{k(d-2)} \gamma_{k}, a_{k}=a \frac{1-L^{-2}}{1-L^{2 k}} \gamma_{k}^{-1}, \lambda_{k}=\lambda L^{2 k} \gamma_{k}, v_{k}=v L^{2 k} \delta_{k}$, depending on renormalization procedures, and the positive constants $\gamma_{k}, \delta_{k}$ satisfy the inequalities

$$
\gamma_{k}, \gamma_{k}^{-1}, \delta_{k}, \delta_{k}^{-1}<\exp \left(c_{8} \sum_{j=1}^{k} L^{-2 \gamma j}\right),
$$

where the positive constant $c_{8}$ can be chosen arbitrarily small if $\beta$ is large enough. Inductive assumptions on $\mathscr{F}_{k}\left(\psi_{k}, h, g\right)$ have a form analogous to the assumptions (H.2)-(H.4), but we formulate them in the one hypothesis below.

(H.7) The function $\mathscr{F}_{k}\left(\psi_{k}, h, g\right)$ has the form

$$
\begin{gathered}
\mathscr{F}_{k}\left(\psi_{k}, h, g\right)=\left\langle g, \phi_{k}\right\rangle_{1}+\sum_{j=1}^{k} \mathscr{F}_{k}^{(j)}\left(\psi_{k}^{(j)}\left(\psi_{k}, h\right), h, g\right), \\
\mathscr{F}_{k}^{(j)}\left(\psi_{j}, h, g\right)=\left\langle g, \mathscr{M}_{k}^{(j)}\left(\psi_{j}, h, g\right)\right\rangle_{1},
\end{gathered}
$$

where the scalar products are taken on the unit lattice $T_{1}$. The functions $\mathscr{M}_{k}^{(j)}\left(x ; \psi_{j}\right.$, $h, g), x \in T_{1}$, are defined and real analytic on the space $\Xi_{j}\left(1, \varepsilon_{j}\right) \times\left\{g:\|g\|_{\ell^{1}}<1\right\}$, and they satisfy the symmetry properties

$$
\begin{gathered}
\mathscr{M}_{k}^{(j)}\left(x ; R \psi_{j}, R h, R g\right)=R \mathscr{M}_{k}^{(j)}\left(x ; \psi_{j}, h, g\right), \quad R \in O(N), \\
\mathscr{M}_{k}^{(j)}\left(r x ; r \psi_{j}, h, r g\right)=\mathscr{M}_{k}^{(j)}\left(x ; \psi_{j}, h, g\right),
\end{gathered}
$$

for all Euclidean transformations $r$ of the lattice $T$ onto itself, which are determined by transformations of the lattice $T^{(j)}$ onto itself. These functions have the localization expansions

$$
\mathscr{M}_{k}^{(j)}\left(x ; \psi_{j}, h, g\right)=\sum_{X \in \mathscr{D}_{j}: x \in X} \mathscr{M}_{k}^{(j)}\left(x, X ; \psi_{j}, h, g\right)
$$

where a term $\mathscr{M}_{k}^{(j)}\left(x, X ; \psi_{j}, h, g\right)$ depends on $\psi_{j}, g$ restricted to $X$, and does not depend on the torus $T$ for $X$ as in (H.4). It has an analytic extension onto the complex space $\Xi_{j}^{c}\left(\mathbb{B}_{j}(X) ; 1, \varepsilon_{j}\right) \times\left\{g: g\right.$ is defined on $T_{1}$ and has values in $\left.\mathbb{C}^{N},\|g\|_{\ell^{1}}<1\right\}$, and the extension depends on configurations $\left(\psi+\psi^{\prime}, h+h^{\prime}, g\right)$ restricted to $X$, hence on $\left(\psi_{j}+\psi_{j}^{\prime}, h+h^{\prime}, g\right)$ restricted to $X$. This extension satisfies the bound

$$
\left|\mathscr{M}_{k}^{(j)}\left(x, X ; \psi_{j}+\psi_{j}^{\prime}, h+h^{\prime}, g\right)\right|<c_{9} \xi^{\frac{d-2}{2}+\gamma-\alpha_{1}} \exp \left(-\kappa d_{j}(X)\right),
$$

where $\kappa$ is as in (H.4), $c_{9}, \alpha_{1}$ are positive constants, which can be chosen arbitrarily small for $\beta$ large enough. It satisfies also the above symmetry properties, with $r$ as in (H.4).

To finish the inductive description let us recall that $\chi_{k}$ is the characteristic function of the space $\Xi_{k}\left(1, \beta_{k}^{-\frac{1}{2}} p\left(\beta_{k}\right)\right)$, where $p(\beta)=A_{0}(\log \beta)^{p_{0}}, p_{0}$ is a fixed even 
integer and $A_{0}$ is a sufficiently large positive constant. Let us notice also that (H.5) can be formulated with $e_{1}$ replaced by an arbitrary vector $h \in S^{N-1}$, and $e_{2}$ replaced by a unit vector orthogonal to $h$, or $e_{2} \otimes e_{2}$ replaced by $I-h \otimes h$. Finally, let us make the important remark that the initial density $\rho_{0}$ defined by $(1.1),(1.5)$ in [1] is not described by the above inductive hypotheses. The way they are formulated presupposes that $k \geqq 1$. We can extend the definitions for $k=0$ taking $Q_{0}=I$ - the identity operator on the lattice $T$, and $\psi_{0}=\phi_{0}$ - the basic spin variable on this lattice, then $\rho_{0}$ satisfies the inductive hypotheses with $\mathscr{E}_{0}\left(\phi_{0}, h\right)=0$, $\mathscr{F}_{0}\left(\phi_{0}, h, g\right)=\left\langle g, \phi_{0}\right\rangle_{1}$. Unfortunately this is not of much help, because the first renormalization transformation applied to $\rho_{0}$ yields the integral (1.13) in [1], which has to be treated in a slightly different way than integrals arising at remaining renormalization transformations, because of the range of the coefficient $\lambda_{0}=\lambda$. We will discuss it in the next section, after a discussion of a general case.

To formulate a main theorem of this paper it is convenient to introduce spaces of densities satisfying the inductive hypotheses. Such a space is determined by the positive integer $k$, the coefficients $\beta, a, \lambda, v$, and the constants $M, \kappa, \alpha_{0}, \alpha_{1}, \gamma, E_{0}, c_{8}, c_{9}$ appearing in the conditions of the hypotheses. Notice that we consider the torus $T$ as one of basic variables on which $\rho_{k}$ depends, so actually we consider families of densities corresponding to all admissible tori as elements of the space. We define

$$
\begin{aligned}
& \mathbb{R}_{k}\left(\beta, a, \lambda, v, B, \bar{v} ; M, \kappa, \alpha_{0}, \alpha_{1}, \gamma, E_{0}, c_{8}, c_{9}\right) \\
& \quad=\left\{\rho_{k}: \rho_{k} \text { satisfies the hypotheses }(\text { H.1 })-(\text { H.7 })\right. \text { determined by }
\end{aligned}
$$

the constants in the parentheses, with $\left.\beta_{k}>B, 0<v_{k} \leqq \bar{v}\right\}$.

Now the main theorem can be written as follows.

Theorem 1. For any constants $M, \kappa, \alpha_{1}, \gamma, c_{8}, c_{9}, \gamma$ from the interval $0<\gamma<$ $\min \left\{\frac{d-2}{2}, \frac{1}{2}\right\}, M, \kappa$ sufficiently large, $\alpha_{1}, c_{8}, c_{9}$ positive and sufficiently small, there exist constants $B, \alpha_{0}, E_{0}$, such that if $\bar{v} \leqq \frac{7}{8} L^{-2}$, then the small field renormalization transformation $\chi_{k+1} S^{(k)} T^{(k)} \chi^{(k)}$ establishes a mapping

$$
\begin{gathered}
\chi_{k+1} S^{(k)} T^{(k)} \chi^{(k)}: \mathbb{R}_{k}\left(\beta, a, \lambda, v, B, \bar{v} ; M, \kappa, \alpha_{0}, \alpha_{1}, \gamma, E_{0}, c_{8}, c_{9}\right) \\
\rightarrow \mathbb{R}_{k+1}\left(\beta, a, \lambda, v, B L^{d-2}, \bar{v} L^{2}, M, \kappa, \alpha_{0}, \alpha_{1}, \gamma, E_{0}, c_{8}, c_{9}\right) .
\end{gathered}
$$

Let us make a few comments on the above theorem. At first notice that its formulation is purely existential, no constructive definitions of "new" coefficients and effective actions are given. In fact the renormalization transformation $\chi_{k+1} S^{(k)} T^{(k)} \chi^{(k)}$ itself does not determine them uniquely, their definitions depend on a renormalization procedure we apply. It would make sense to introduce a third operation following $S^{(k)}, T^{(k)}$, and corresponding to the renormalization procedure, because only this defines uniquely all elements of "new" densities. Unfortunately a definition of such an operation would be very complicated, practically impossible without going into many complicated details of the whole procedure, so we have decided not to introduce it here, it is buried in the existential statement of the theorem. Of course a detailed and precise description of the renormalization procedure is given later in the paper, in fact two sections are devoted to it. Notice also that we have not yet defined the characteristic function $\chi^{(k)}$. It is a very simple function restricting fluctuation variables, but a definition of these variables is quite technical, involving some operators which appear later in the paper. We could introduce all these definitions 
here, but it is much more natural to postpone the definition of $\chi^{(k)}$ to a proper place later. Finally let us remark that the transformation (1.5) is obviously a non-linear one, it depends in a complicated non-linear manner on various elements of densities $\rho_{k}$, in particular on $\beta_{k}$, and on "new" constants $\beta_{k+1}$ through the characteristic function $\chi_{k+1}$.

Densities $\rho_{k}$ in a space (1.4) are uniquely determined by coefficients $\beta_{k}, a_{k}, \lambda_{k}, v_{k}$ and functions $\mathscr{E}_{k}^{(j)}, \mathscr{F}_{k}^{(j)}, j=1, \ldots, k$. We define spaces of these elements

$$
\begin{aligned}
& \mathbb{A}_{k}\left(\beta, a, \lambda, v, B, \bar{v} ; M, \kappa, \alpha_{0}, \alpha_{1}, \gamma, E_{0}, c_{8}, c_{9}\right) \\
& =\left\{\left(\beta_{k}, a_{k}, \lambda_{k}, v_{k},\left(\mathscr{E}_{k}^{(j)}\right)_{j \leqq k},\left(\mathscr{M}_{k}^{(j)}\right)_{j \leqq k}\right): \beta_{k}, a_{k}, \lambda_{k}, v_{k}\right. \text { satisfy (H.1), (H.6), } \\
& \left.\quad \text { and } \beta_{k}>B, 0<v_{k} \leqq \bar{v}, \mathscr{E}_{k}^{(j)} \text { satisfy (H.2)-(H.5), } \mathscr{M}_{k}^{(j)} \text { satisfy (H.7) }\right\} .
\end{aligned}
$$

The conditions (H.2)-(H.5), and (H.7), except the bounds in (H.4), (H.7), define real vector spaces of the functions $\left(\mathscr{E}_{k}^{(j)}\right)_{j \leqq k}$, and $\left(\mathscr{M}_{k}^{(j)}\right)_{j \leqq k}$. The bounds define open domains in these spaces, which are open balls with respect to appropriate norms. The conditions in (H.1), (H.6) define a closure of an open domain in a four-dimensional real vector space of the coefficients $\beta_{k}, a_{k}, \lambda_{k}, v_{k}$. Because of the one-to-one mapping between the spaces (1.4) and (1.6) the renormalization transformation (1.5) defines a mapping between appropriate spaces (1.6). We reformulate Theorem 1 in a more precise way.

Theorem 2. Under the assumptions of Theorem 1 the small field renormalization transformation establishes a real analytic mapping

$$
\begin{aligned}
& \mathscr{S}^{(k)} \mathscr{T}^{(k)}: \mathbb{A}_{k}\left(\beta, a, \lambda, v, B, \bar{v} ; M, \kappa, \alpha_{0}, \alpha_{1}, \gamma, E_{0}, c_{8}, c_{9}\right) \\
& \quad \rightarrow \mathbb{A}_{k+1}\left(\beta, a, \lambda, v, B L^{d-2}, \bar{v} L^{2} ; M, \kappa, \alpha_{0}, \alpha_{1}, \gamma, E_{0}, c_{8}, c_{9}\right) .
\end{aligned}
$$

$A$ value of the mapping on an element of the first space is an element $\left(\beta_{k+1}, a_{k+1}\right.$, $\left.\lambda_{k+1}, v_{k+1},\left(\mathscr{E}_{k+1}^{(j)}\right)_{j \leqq k+1},\left(\mathscr{M}_{k+1}^{(j)}\right)_{j \leqq k+1}\right)$ of the second space, for which the following equations are satisfied:

$$
\begin{gathered}
\mathscr{E}_{k+1}^{(j)}=\mathscr{E}_{k}^{(j)}, \mathscr{M}_{k+1}^{(j)}=\mathscr{M}_{k}^{(j)} \quad \text { for } j \leqq k \\
\beta_{k+1}=\beta_{k} L^{d-2}-b_{k+1}, \quad a_{k+1}=\frac{\beta_{k} L^{d-2}}{\beta_{k+1}} \frac{a a_{k}}{a L^{-2}+a_{k}}-\frac{c_{k+1}}{\beta_{k+1}}, \\
\lambda_{k+1}=\lambda_{k} L^{2} \frac{\beta_{k+1}+d_{k+1}}{\beta_{k} L^{d-2}}=\lambda_{k} L^{2} \frac{\beta_{k} L^{d-2}-b_{k+1}+d_{k+1}}{\beta_{k} L^{d-2}} \\
v_{k+1}=\frac{\beta_{k} L^{d-2}}{\beta_{k+1}+e_{k+1}} v_{k} L^{2}=\frac{\beta_{k+1}+b_{k+1}}{\beta_{k+1}+e_{k+1}} v_{k} L^{2},
\end{gathered}
$$

where the constants $b_{k+1}, c_{k+1}, d_{k+1}, e_{k+1}$ are real analytic functions of $\beta_{k}, a_{k}, \lambda_{k}, v_{k}$, $\left(\mathscr{E}_{k}^{(j)}\right)_{j \leqq k}$. They are small compared with $\beta_{k} L^{d-2}$ or $\beta_{k+1}$, more precisely

$$
\left|\beta_{k+1}^{-1} b_{k+1}\right|,\left|\beta_{k+1}^{-1} c_{k+1}\right|,\left|\beta_{k+1}^{-1} d_{k+1}\right|,\left|\beta_{k+1}^{-1} e_{k+1}\right|<\frac{1}{4} c_{8} L^{-(k+1) 2 \gamma}
$$

for $B$ large enough. 
The formulation of the above theorem requires several comments. The first concerns the equalities (1.8). The functions on both sides have different domains, because the spaces $\Xi_{j}\left(1, \varepsilon_{j}\right)$ depend in fact on $k$ through the constant $\alpha_{0, k}$ and the coefficients $a_{j}, \lambda_{j}, v_{j}$, which are connected with $a_{k}, \lambda_{k}, v_{k}$ by the equalities written in (H.2). These spaces decrease when $k$ increases, so the domains of the functions on the left-hand sides of (1.9) are contained in the domains of the functions on the right-hand sides, and the functions with the subscript " $k+1$ " are restrictions of the functions with the subscript " $k$." Because of this property we drop the subscripts from the symbols of these functions from now on, so we denote them simply by $\mathscr{E}^{(j)}, \mathscr{F}^{(j)}$, as in [1]. This property is very important, it means that the "new" action $\mathscr{E}_{k+1}$ determined by the renormalization transformation can be obtained from the "old" one $\mathscr{E}_{k}$ replacing the functions $\psi_{k}^{(j)}$ in (H.2) by $\psi_{k+1}^{(j)}$, and adding a new contribution $\mathscr{E}^{(k+1)}$. The same is true for $\mathscr{F}_{k+1}$. The second comment is about the renormalization group equations (1.9). The corrections with $b_{k+1}, c_{k+1}$, etc. are called renormalizations. The coefficients must be renormalized in order to create new terms, which are included into the new contribution $\mathscr{E}^{(k+1)}$, so that it satisfies the renormalization conditions (H.5). This can be done in many different ways; there is no uniqueness in the renormalization procedure. We analyze here a class of possible procedures leading to the class (1.9) of renormalization group equations with some additional restrictions. The third comment concerns the constants $E_{k}$ in (H.1). We have not included them in the above theorem because they do not play any role in the small field analysis. They are included in Theorem 1, and they change in a simple way with each renormalization step, which is precisely described later on, but the description is not included in the formulation of the inductive hypotheses and the theorem. The final comment is about the real analyticity of the mapping (1.7). This property does not play any role in the method, it follows simply from the fact that the mapping is given by explicit, though quite complicated expressions, which can be extended analytically in obvious ways to a complex small neighborhood of real actions. We will repeat this statement in proper places in the text, but it will always be obvious.

The above theorem implies Theorem 1 , even in a more precise form. The two theorems imply the fundamental Theorem 2.2 in [1].

Let us describe now briefly contents of subsequent sections. In Sect. 2 we reduce the renormalization transformation $T^{(k)}$ to a fluctuation integral, which is a small perturbation of a simple Gaussian integral, and we define various contributions to a "new" action. In the very short Sect. 3 we perform the scaling operation $S^{(k)}$, and we summarize results obtained until then. In Sect. 4 we describe a renormalization procedure of the effective action, or rather of the new contributions to the action. In Sect. 5 we discuss resulting renormalization group equations for new coefficients. This completes the proof of the two theorems. The paper ends with the short Sect. 6, where a last step in this renormalization group approach is discussed together with some simple conclusions. In the whole paper we freely use notations and results of the three preceding papers $[1,2,3]$, making references only for most important definitions and theorems.

Let us stress again that constructions and results of this paper, although formulated for the case when the whole lattice is a small field domain, are valid in a most general case with an arbitrary admissible small field domain. 


\section{The Renormalization Transformation $T^{(k)}$ and a Fluctuation Integral}

We take a density $\rho_{k}$ from a space (1.4), and we apply the renormalization transformation $T^{(k)} \chi^{(k)}$,

$$
\begin{aligned}
&\left(T^{(k)} \chi^{(k)} \rho_{k}\right)(\theta)= \int d \psi t\left(\theta, \psi ; \beta_{k}, a\right) \chi^{(k)} \chi_{k} \exp \left[\mathscr{A}_{k}(\psi)+\mathscr{F}_{k}(\psi, g)\right] \\
&= \int d \psi \chi^{(k)} \chi_{k} \exp \left[-\beta_{k}\left\{\frac{1}{2} a L^{-2}\|\theta-Q \psi\|_{L}^{2}+A_{k}\left(\psi, \phi_{k}\right)\right\}\right. \\
&\left.\quad+\mathscr{E}_{k}(\psi)-E_{k}^{\prime}\left|T_{1}^{(k)}\right|+\mathscr{F}_{k}(\psi, g)\right], \\
& E_{k}^{\prime}=E_{k}-\frac{1}{2} L^{-d} N \log \frac{\beta_{k} a L^{-2}}{2 \pi} .
\end{aligned}
$$

The characteristic function $\chi^{(k)}$ is a product of two functions, one is equal to

$$
\chi\left(\left\{|\theta-Q \psi|<2 \beta_{k}^{-\frac{1}{2}} p\left(\beta_{k}\right)\right\}\right)
$$

and another is a characteristic function introducing restrictions on fluctuation variables, which will be defined later on. Let us remark that the spin configuration $\theta$ is not equal to the new spin configuration $\psi_{k+1}$, in fact they are connected by a scaling transformation determined later. We use the variable $\theta$ at this stage to simplify notations. For the same reason we write $\psi$ instead of $\psi_{k}$. Consider now the function of $\psi$ in the exponential in (2.1). It is a sum of two terms, one is the function in the curly brackets multiplied by the large parameter $\beta_{k}$, another is the remaining bounded in $\beta_{k}$ function of $\psi$. In such a case it is natural to apply the saddle point method to "calculate" and estimate the integral. A first step of the method is to find critical points of the function in the curly brackets. This problem has been analyzed thoroughly in Sect. 4 of [2]. To apply the results of that section we have to understand restrictions on variables $\theta, \psi$ implied by the characteristic functions in (2.1). The characteristic function $\chi_{k}$ implies that configurations $\psi$ in the domain of integration belong to the space $\tilde{\Psi}_{k}\left(3 \beta_{k}^{-\frac{1}{2}} p\left(\beta_{k}\right)\right)$, by Lemma 3.1 of [1]. Repeating some arguments from the proof of this lemma we see easily that the restrictions defining the space $\tilde{\Psi}_{k}\left(3 \beta_{k}^{-\frac{1}{2}} p\left(\beta_{k}\right)\right)$ together with the restrictions defining the characteristic function (2.2) imply the following ones:

$$
\begin{gathered}
\left|\partial^{1} \theta\right|<(3 L+4) \beta_{k}^{-\frac{1}{2}} p\left(\beta_{k}\right),\left|\theta^{2}-1\right|<8 \beta_{k}^{-\frac{1}{2}} p\left(\beta_{k}\right), \\
v_{k}\left(1-h \cdot \theta_{0}\right)<12^{2} \beta_{k}^{-1} p^{2}\left(\beta_{k}\right),
\end{gathered}
$$

assuming that $\beta_{k}$ is sufficiently large. These conditions imply that $\theta \in \tilde{\Psi}_{k}\left(\delta_{1}\right)$ with $\delta_{1}=4 L \beta_{k}^{-\frac{1}{2}} p\left(\beta_{k}\right)$. If $\delta_{1}$ is sufficiently small, then we can apply Proposition 4.1 from [2] and conclude that the function in the curly brackets in (2.1), considered on the space $\tilde{\Psi}_{k}\left(L^{-2} c_{1}\right)$, has exactly one critical point, which is a minimum of the function. Keeping the notations of [2] we should denote this minimal configuration by $\psi_{k+1}^{(k)}$, but for simplicity, we drop the subscript and denote it by $\psi^{(k)}$. Let us write the corresponding equations and expansions around their solutions, because we need 
them to study the effective action in the integral (2.1). We have

$$
\frac{1}{2} a L^{-2}\|\theta-Q \psi\|_{L}^{2}+A_{k}\left(\psi, \phi_{k}\right)=J_{k}\left(\theta, \psi, \phi_{k}, \alpha_{k}\right)
$$

where

$$
\begin{gathered}
J_{k}(\theta, \psi, \phi, \alpha)=\frac{1}{2} a L^{-2}\|\theta-Q \psi\|_{L}^{2}+A_{k}(\psi, \phi, \alpha) \\
A_{k}(\psi, \phi, \alpha)=\frac{1}{2} a_{k}\left\|\psi-Q_{k} \phi\right\|^{2}+\frac{1}{2}\left\|\partial^{\eta} \phi\right\|^{2}+\frac{1}{2}\left\langle\alpha,|\phi|^{2}-1\right\rangle \\
-\frac{1}{2 \lambda_{k}}\|\alpha\|^{2}+\frac{1}{2} v_{k}\|\phi-h\|^{2}
\end{gathered}
$$

We have then

$$
\inf _{\psi} J_{k}\left(\theta, \psi, \phi_{k}(\psi), \alpha_{k}(\psi)\right)=\inf _{\psi} \inf _{\phi} \sup _{\alpha} J_{k}(\theta, \psi, \phi, \alpha) .
$$

It follows that if $\psi_{0}$ is a critical point of the function (2.4), then $\left(\psi_{0}, \phi_{k}\left(\psi_{0}\right), \alpha_{k}\left(\psi_{0}\right)\right)$ is a critical point of the function (2.5), and it satisfies the following variational equations:

$$
\begin{gathered}
\frac{\delta}{\delta \psi} J_{k}(\theta, \psi, \phi, \alpha)=a L^{-2} Q^{*}(Q \psi-\theta)+a_{k}\left(\psi-Q_{k} \phi\right)=0 \\
\frac{\delta}{\delta \phi} J_{k}(\theta, \psi, \phi, \alpha)=a_{k} Q_{k}^{*}\left(Q_{k} \phi-\psi\right)-\Delta^{\eta} \phi+\alpha \phi+v_{k}(\phi-h)=0, \\
\frac{\delta}{\delta \alpha} J_{k}(\theta, \psi, \phi, \alpha)=\frac{1}{2}\left(|\phi|^{2}-1\right)-\frac{1}{\lambda_{k}} \alpha=0 .
\end{gathered}
$$

The function $J_{k}$ and the equations are defined for all $\theta, \psi, \phi, \alpha$, and the first two equations are linear in $\psi, \phi$. The first equation has a unique solution for $\psi$ without any restrictions on $\theta, \phi$, and we get

$$
\psi=Q_{k} \phi-\frac{a L^{-2}}{a_{k}+a L^{-2}} Q^{*} Q_{k+1} \phi+\frac{a L^{-2}}{a_{k}+a L^{-2}} Q^{*} \theta .
$$

Of course the equation and the solution are connected with calculating the composition of the renormalization transformations $T^{(k)}$ and $T_{k}$. Substituting the solution into the second equation and defining

$$
a_{k+1}=\frac{a_{k} a}{a_{k}+a L^{-2}},
$$

which is the second equation (1.10), we obtain the following system of equations for $\phi, \alpha$ :

$$
\begin{gathered}
\left(-\Delta^{\eta}+a_{k+1} L^{-2} Q_{k+1}^{*} Q_{k+1}+\alpha\right) \phi=a_{k+1} L^{-2} Q_{k+1}^{*} \theta+v_{k} h, \\
\frac{1}{2}\left(|\phi|^{2}-1\right)-\frac{1}{\lambda_{k}} \alpha=0 .
\end{gathered}
$$


If we calculate $\alpha$ from the second equation, substitute it into the first, and then multiply the obtained equation by $L^{2}$, we obtain the variational equation for the problem of finding

$$
\inf _{\phi} A_{k+1}\left(\theta, \phi ; h, a_{k+1}, \lambda_{k} L^{2}, v_{k} L^{2}\right) \text {. }
$$

Denoting $\lambda_{k+1}=\lambda_{k} L^{2}, v_{k+1}=v_{k} L^{2}$, which are extensions of the equalities in (H.2), we have the basic variational problem defining the minimal configurations $\phi_{k+1}$ on the natural scale $L^{-1} \eta$. They are defined by the unrenormalized coefficients $a_{k+1}, \lambda_{k+1}, v_{k+1}$, so in the future we will write also the coefficients and the minimal configurations with the subscript $u$. On the $\eta$-scale, the configurations are determined by Eqs. (2.10) with the coefficients $a_{k+1} L^{-2}, \lambda_{k}, v_{k}$, so we have the scaling property

$$
\phi_{k+1}\left(L^{-1} x ; \theta, h, a_{k+1}, \lambda_{k+1}, v_{k+1}\right)=\phi_{k+1}\left(x ; \theta, h, a_{k+1} L^{-2}, \lambda_{k}, v_{k}\right), \quad x \in T_{\eta} .
$$

The function $\phi_{k+1}(\theta)$ is defined if $(\theta, h) \in \tilde{\Psi}_{k+1}\left(c_{1}\right)$, hence if $(\theta, h) \in \tilde{\Psi}_{k}\left(L^{-2} c_{1}\right)$, because the last two conditions in the definition (3.18) [1] of these spaces involve the constant $v_{k}=v_{k+1} L^{-2}$. This is satisfied if $\beta_{k}^{-\frac{1}{2}} p\left(\beta_{k}\right)$ is sufficiently small, or $\beta_{k}$ sufficiently large. Then the solution of the variational problem on the left-hand side of (2.6) is obtained by substituting $\phi_{k+1}$ instead of $\phi$ in the formula (2.8), and we have

$$
\psi^{(k)}(\theta)=Q_{k} \phi_{k+1}(\theta)+\frac{a_{k+1}}{a_{k}} L^{-2} Q^{*}\left(\theta-Q_{k+1} \phi_{k+1}(\theta)\right) .
$$

We conclude that the system of Eqs. (2.7) has a solution if $(\theta, h) \in \tilde{\Psi}_{k+1}\left(c_{1}\right)$. The solution is unique in the space of all functions $\psi, \phi, \alpha$ satisfying only the condition $|\alpha|<c_{0} L^{-2}$, and is given by the configurations $\psi^{(k)}(\theta), \phi_{k+1}(\theta), \alpha_{k+1}(\theta)$. They satisfy the composition formulas

$$
\phi_{k}\left(\psi^{(k)}(\theta)\right)=\phi_{k+1}(\theta), \quad \alpha_{k}\left(\psi^{(k)}(\theta)\right)=\alpha_{k+1}(\theta)
$$

holding for $\theta$ such that $\psi^{(k)}(\theta)$ is in the domain of the function $\phi_{k}(\psi)$. This holds certainly if $\beta_{k}$ is sufficiently large, by Proposition 4.1 [2].

Let us study expansions of the functions $\phi_{k}, \alpha_{k}$ and the action $J_{k}$ around the minimal configuration $\psi^{(k)}$. We write

$$
\psi=\psi^{(k)}+\psi^{\prime}, \quad \phi_{k}\left(\psi^{(k)}+\psi^{\prime}\right)=\phi_{k+1}+\phi^{\prime}, \quad \alpha_{k}\left(\psi^{(k)}+\psi^{\prime}\right)=\alpha_{k+1}+\alpha^{\prime},
$$

and we substitute the above equalities into $J_{k}$. We get

$$
\begin{aligned}
J_{k}\left(\theta, \psi, \phi_{k}(\psi), \alpha_{k}(\psi)\right)= & A_{k+1}^{\eta}\left(\theta, \phi_{k+1}\right)+\frac{1}{2} a L^{-2}\left\|Q \psi^{\prime}\right\|_{L}^{2}+\frac{1}{2} a_{k}\left\|\psi^{\prime}-Q_{k} \phi^{\prime}\right\|^{2} \\
& +\frac{1}{2}\left\langle\phi^{\prime},\left(-\Delta^{\eta}+\alpha_{k+1}+v_{k}\right) \phi^{\prime}\right\rangle \\
& +\left\langle\alpha^{\prime}, \phi_{k+1} \cdot \phi^{\prime}+\frac{1}{2}\left|\phi^{\prime}\right|^{2}\right\rangle-\frac{1}{2 \lambda_{k}}\left\|\alpha^{\prime}\right\|^{2}
\end{aligned}
$$

where we have used the variational equations (2.7) satisfied by the configurations $\psi^{(k)}, \phi_{k+1}, \alpha_{k+1}$. We determine the functions $\phi^{\prime}, \alpha^{\prime}$ by solving the variational problem of taking a supremum over $\alpha^{\prime}$ and an infimum over $\phi^{\prime}$ of the function of $\phi^{\prime}, \alpha^{\prime}$ on 
the right-hand side above. The corresponding variational equations are

$$
\begin{gathered}
\left(-\Delta^{\eta}+a_{k} Q_{k}^{*} Q_{k}+v_{k}+\alpha_{k+1}+\alpha^{\prime}\right) \phi^{\prime}=a_{k} Q_{k}^{*} \psi^{\prime}-\phi_{k+1} \alpha^{\prime}, \\
\phi_{k+1} \cdot \phi^{\prime}+\frac{1}{2}\left|\phi^{\prime}\right|^{2}-\frac{1}{\lambda_{k}} \alpha^{\prime}=0 .
\end{gathered}
$$

These equations are special cases of Eqs. (3.19), or (3.20) in [2], with $\delta \psi=\psi^{\prime}, \phi_{0}=$ $\phi_{k+1}, \alpha_{0}=\alpha_{k+1}, f_{1}=0, \alpha_{1}=0$. Applying Proposition 3.1 of [2] we obtain that there exists exactly one solution, denoted by $\delta \phi_{k}, \delta \alpha_{k}$, if $\left|\psi^{\prime}\right|<K_{2}^{-1} c_{6}$ and $\beta_{k}$ is large enough. This solution is an analytic function of $\psi^{\prime}, \phi_{k+1}, \alpha_{k+1}$, and it satisfies the bounds

$$
\left|\delta \phi_{k}\left(\psi^{\prime}\right)\right|,\left|\partial^{\eta} \delta \phi_{k}\left(\psi^{\prime}\right)\right|,\left|\Delta^{\eta} \delta \phi_{k}\left(\psi^{\prime}\right)\right|,\left|\delta \alpha_{k}\left(\psi^{\prime}\right)\right| \leqq K_{2}\left|\psi^{\prime}\right| .
$$

These bounds will be used several times in the future. They show that the analytic functions $\delta \phi_{k}\left(\psi^{\prime}\right), \delta \alpha_{k}\left(\psi^{\prime}\right)$ are at least of first order in $\psi^{\prime}$. Substituting the solutions into the function on the right-hand side of (2.16) we obtain

$$
\begin{aligned}
J_{k}\left(\theta, \psi, \phi_{k}(\psi), \alpha_{k}(\psi)\right)= & A_{k+1}^{\eta}\left(\theta, \phi_{k+1}\right)+\frac{1}{2} a L^{-2}\left\|Q \psi^{\prime}\right\|_{L}^{2}+\frac{1}{2} a_{k}\left\|\psi^{\prime}-Q_{k} \delta \phi_{k}\left(\psi^{\prime}\right)\right\|^{2} \\
& +\frac{1}{2}\left\langle\delta \phi_{k}\left(\psi^{\prime}\right),\left(-\Delta^{\eta}+v_{k}+\alpha_{k+1}\right) \delta \phi_{k}\left(\psi^{\prime}\right)\right\rangle \\
& +\frac{1}{2 \lambda_{k}}\left\|\delta \alpha_{k}\left(\psi^{\prime}\right)\right\|^{2}
\end{aligned}
$$

where we have used the second equation (2.17) to simplify the expression. To find out the quadratic form in $\psi^{\prime}$ we have to find linear terms in expansions of $\delta \phi_{k}\left(\psi^{\prime}\right), \delta \alpha_{k}\left(\psi^{\prime}\right)$, and to substitute them into the above expression. Notice that it is quadratic in those functions. Let us write

$$
\delta \phi_{k}\left(\psi^{\prime}\right)=\delta \phi_{k}^{(1)}\left(\psi^{\prime}\right)+\delta \phi_{k, 2}\left(\psi^{\prime}\right), \quad \delta \alpha_{k}\left(\psi^{\prime}\right)=\delta \alpha_{k}^{(1)}\left(\psi^{\prime}\right)+\delta \alpha_{k, 2}\left(\psi^{\prime}\right),
$$

where the functions $\delta \phi_{k}^{(1)}\left(\psi^{\prime}\right), \delta \alpha_{k}^{(1)}\left(\psi^{\prime}\right)$ are linear in $\psi^{\prime}$, and $\delta \phi_{k, 2}\left(\psi^{\prime}\right), \delta \alpha_{k, 2}\left(\psi^{\prime}\right)$ are analytic and at least of second order in $\psi^{\prime}$. The linear parts can be found from the corresponding linear variational equations

$$
\begin{aligned}
\left(-\Delta^{\eta}+a_{k} Q_{k}^{*} Q_{k}+v_{k}+\alpha_{k+1}\right) \phi^{\prime} & =a_{k} Q_{k}^{*} \psi^{\prime}-\phi_{k+1} \alpha^{\prime}, \\
\phi_{k+1} \cdot \phi^{\prime}-\frac{1}{\lambda_{k}} \alpha^{\prime} & =0 .
\end{aligned}
$$

A solution of the first equation is

$$
\phi^{\prime}=G_{k}\left(\alpha_{k+1}\right) a_{k} Q_{k}^{*} \psi^{\prime}-G_{k}\left(\alpha_{k+1}\right) \phi_{k+1} \alpha^{\prime} .
$$

Substituting it into the second and solving it we get

$$
\alpha^{\prime}=\left[\frac{1}{\lambda_{k}}+\phi_{k+1} \cdot G_{k}\left(\alpha_{k+1}\right) \phi_{k+1}\right]^{-1} \phi_{k+1} \cdot G_{k}\left(\alpha_{k+1}\right) a_{k} Q_{k}^{*} \psi^{\prime} .
$$

The operator in the square bracket above and its inverse have been analyzed in detail in paper [2]. The inverse is basically a bounded perturbation of the operator $-\Delta^{\eta}$, 
and the above linear operator is bounded and has an exponential decay property. The function in (2.22) is equal to $\delta \alpha_{k}^{(1)}\left(\psi^{\prime}\right)$, and substituting it instead of $\alpha^{\prime}$ in (2.21) we get $\delta \phi_{k}^{(1)}\left(\psi^{\prime}\right)$. Substituting these linear functions into the expression in (2.19), we obtain the quadratic form

$$
\begin{aligned}
& \frac{1}{2}\left\langle\psi^{\prime}, \Delta^{(k)} \psi^{\prime}\right\rangle=\frac{1}{2} a_{k}\left\|\psi^{\prime}-Q_{k} \delta \phi_{k}^{(1)}\left(\psi^{\prime}\right)\right\|^{2} \\
& +\frac{1}{2}\left\langle\delta \phi_{k}^{(1)}\left(\psi^{\prime}\right),\left(-\Delta^{\eta}+v_{k}+\alpha_{k+1}\right) \delta \phi_{k}^{(1)}\left(\psi^{\prime}\right)\right\rangle+\frac{1}{2 \lambda_{k}}\left\|\delta \alpha_{k}^{(1)}\left(\psi^{\prime}\right)\right\|^{2} \\
& =\frac{1}{2} a_{k}\left\|\psi^{\prime}\right\|^{2}-\frac{1}{2} a_{k}^{2}\left\langle\psi^{\prime}, Q_{k} G_{k}\left(\alpha_{k+1}\right) Q_{k}^{*} \psi^{\prime}\right\rangle \\
& +\frac{1}{2} a_{k}^{2}\left\langle\phi_{k+1} \cdot G_{k}\left(\alpha_{k+1}\right) Q_{k}^{*} \psi^{\prime},\left[\frac{1}{\lambda_{k}}+\phi_{k+1} \cdot G_{k}\left(\alpha_{k+1}\right) \phi_{k+1}\right]^{-1} \phi_{k+1}\right. \\
& \left.\cdot G_{k}\left(\alpha_{k+1}\right) Q_{k}^{*} \psi^{\prime}\right\rangle
\end{aligned}
$$

The operator of this quadratic form is a unit lattice bounded operator with exponentially decaying matrix elements, the bound and the decay rate are uniform in $k$. The last term on the right-hand side above is non-negative, so we have the bound

$$
\frac{1}{2}\left\langle\psi^{\prime}, \Delta^{(k)} \psi^{\prime}\right\rangle \geqq \frac{1}{2} a_{k}\left\|\psi^{\prime}\right\|^{2}-\frac{1}{2} a_{k}^{2}\left\langle\psi^{\prime}, Q_{k} G_{k} Q_{k}^{*} \psi^{\prime}\right\rangle-O\left(\left|\alpha_{k+1}\right|\right)\left\|\psi^{\prime}\right\|^{2},
$$

where $G_{k}=G_{k}(0)$, and we have expanded $G_{k}\left(\alpha_{k+1}\right)$ up to the first order in $\alpha_{k+1}$. The first two terms on the right-hand side above define a quadratic form which is equal to the effective quadratic form obtained by applying $k$ times the Gaussian renormalization transformation $T_{a}$ to the Gaussian density determined by the quadratic form $\frac{1}{2}\left\langle\phi,\left(-\Delta+v_{k} L^{-2 k}\right) \phi\right\rangle$. These effective forms have been investigated thoroughly in many papers, e.g. in [2c] it has been shown that they are bounded from below by $\frac{1}{2} \gamma_{0}\left\langle\psi^{\prime},\left(-\Delta+v_{k}\right) \psi^{\prime}\right\rangle$ uniformly in $k$. From this and the bound (2.24) we obtain that the quadratic form in the expression on the right-hand side of (2.19) satisfies the bound

$$
\frac{1}{2} a L^{-2}\left\|Q \psi^{\prime}\right\|_{L}^{2}+\frac{1}{2}\left\langle\psi^{\prime}, \Delta^{(k)} \psi^{\prime}\right\rangle \geqq \frac{1}{2} \gamma_{0} L^{-2}\left\|\psi^{\prime}\right\|^{2}
$$

with the absolute positive constant $\gamma_{0}$. Thus the operator of this form satisfies all the assumptions of the theorem in Sect. 5 of [3] on unit lattice operators, hence its inverse has a uniform exponential decay. This inverse is denoted by $C^{(k)}$ and it is a covariance of the corresponding Gaussian measure. It can be expressed explicitly in terms of operators like $G_{k}$, which are some bounded functions of $-\Delta^{\eta}$. Actually of more interest to us are various localization expansions constructed in [3], but these will be discussed in a following paper. All the above operators depend also analytically on the configurations $\phi_{k+1}, \alpha_{k+1}$. Let us write now the expansion (2.19) 
separating explicitly the quadratic form in $\psi^{\prime}$,

$J_{k}\left(\theta, \psi, \phi_{k}(\psi), \alpha_{k}(\psi)\right)=A_{k+1}^{\eta}\left(\theta, \phi_{k+1}\right)+\frac{1}{2} a L^{-2}\left\|Q \psi^{\prime}\right\|^{2}+\frac{1}{2}\left\langle\psi^{\prime}, \Delta^{(k)} \psi^{\prime}\right\rangle+V^{(k)}\left(\psi^{\prime}\right)$,

where

$$
\begin{aligned}
V^{(k)}\left(\psi^{\prime}\right)= & -a_{k}\left\langle\psi^{\prime}-Q_{k} \delta \phi_{k}^{(1)}\left(\psi^{\prime}\right), Q_{k} \delta \phi_{k, 2}\left(\psi^{\prime}\right)\right\rangle \\
& +\frac{1}{2} a_{k}\left\|Q_{k} \delta \phi_{k, 2}\left(\psi^{\prime}\right)\right\|^{2}+\left\langle\delta \phi_{k}^{(1)}\left(\psi^{\prime}\right),\left(-\Delta+v_{k}+\alpha_{k+1}\right) \delta \phi_{k, 2}\left(\psi^{\prime}\right)\right\rangle \\
& +\frac{1}{2}\left\langle\delta \phi_{k, 2}\left(\psi^{\prime}\right),\left(-\Delta+v_{k}+\alpha_{k+1}\right) \delta \phi_{k, 2}\left(\psi^{\prime}\right)\right\rangle \\
& +\frac{1}{\lambda_{k}}\left\langle\delta \alpha_{k}^{(1)}\left(\psi^{\prime}\right), \delta \alpha_{k, 2}\left(\psi^{\prime}\right)\right\rangle+\frac{1}{2 \lambda_{k}}\left\|\delta \alpha_{k, 2}\left(\psi^{\prime}\right)\right\|^{2}
\end{aligned}
$$

The above expression is an analytic function of $\psi^{\prime}, \phi_{k+1}, \alpha_{k+1}$, invariant with respect to transformations $R \in O(N)$ of the configurations $\psi^{\prime}, \phi_{k+1}$, and invariant with respect to Euclidean transformations $r$ of the configurations $\psi^{\prime}, \phi_{k+1}, \alpha_{k+1}$, which transform the lattice $T^{(k+1)}$ onto itself. This is true because the effective actions and the variational equations have the corresponding invariance or covariance properties. An important property of the function $V^{(k)}$ is its localizability. This function is a sum of terms which are defined by scalar products on corresponding lattices $T_{1}^{(k)}$ and $T_{\eta}$. Let us define a function $V^{(k)}(z), z \in T_{L}^{(k+1)}$, restricting the scalar products to points of the block $B_{k+1}(z)$. We have

$$
V^{(k)}\left(\psi^{\prime}\right)=\sum_{z \in T_{L}^{(k+1)}} V^{(k)}\left(z ; \psi^{\prime}\right),
$$

and the functions $V^{(k)}(z)$ have the following symmetry properties:

$$
V^{(k)}\left(z ; R \psi^{\prime}, R \phi_{k+1}, \alpha_{k+1}\right)=V^{(k)}\left(z ; \psi^{\prime}, \phi_{k+1}, \alpha_{k+1}\right)
$$

for orthogonal transformations $R \in O(N)$,

$$
V^{(k)}\left(r z ; r \psi^{\prime}, r \phi_{k+1}, r \alpha_{k+1}\right)=V^{(k)}\left(z ; \psi^{\prime}, \phi_{k+1}, \alpha_{k+1}\right)
$$

for Euclidean transformations $r$ mapping $T^{(k+1)}$ onto itself. The functions $V^{(k)}(z)$ are obviously analytic functions of $\psi^{\prime}, \phi_{k+1}, \alpha_{k+1}$, at least of third order in $\psi^{\prime}$, i.e. $V^{(k)}\left(z ; \psi^{\prime}\right)=O\left(\left|\psi^{\prime}\right|^{3}\right)$. A crucial property of $V^{(k)}\left(z ; \psi^{\prime}, \phi_{k+1}, \alpha_{k+1}\right)$ is that it has a localization expansion with an exponential tree decay property in localization domains of the type described in (H.4). This will be constructed in the following paper.

Let us come back to the analysis of the integral in (2.1). We have noticed already that the configuration $\theta$ satisfies the restrictions (2.3), so the function $J_{k}\left(\theta, \psi, \phi_{k}(\psi), \alpha_{k}(\psi)\right)$ in the curly bracket in (2.1) has exactly one minimum $\psi^{(k)}(\theta)$ in the space $\tilde{\Psi}_{k}\left(c_{1}\right)$. In general the configuration $\psi^{(k)}(\theta)$ may not belong to the domain of integration in (2.1) determined by the characteristic functions. To assure that it does belong to this domain we impose new small field restrictions on $\theta$ 
introducing the characteristic function

$$
\begin{gathered}
\chi_{k+1, u}=\chi\left(\left\{\left|\theta-Q_{k+1} \phi_{k+1}\right|<\frac{3}{2} \beta_{k}^{-\frac{1}{2}} p\left(\beta_{k}\right),\left|\partial^{\eta} \phi_{k+1}\right|<\frac{1}{2} \beta_{k}^{-\frac{1}{2}} p\left(\beta_{k}\right),\right.\right. \\
\left|\Delta^{\eta} \phi_{k+1}\right|<\frac{1}{2} \beta_{k}^{-\frac{1}{2}} p\left(\beta_{k}\right),\left|\alpha_{k+1}\right|<\frac{1}{2} \beta_{k}^{-\frac{1}{2}} p\left(\beta_{k}\right), \\
\left.\left.\left|\phi_{k+1}-h\right|<\frac{1}{2} v_{k}^{-\frac{1}{2}} \beta_{k}^{-\frac{1}{2}} p\left(\beta_{k}\right)\right\}\right) .
\end{gathered}
$$

The subscript $u$ here means that the function is defined in terms of unrenormalized configurations and coefficients. The restrictions above together with the composition formulas (2.14) and the formula (2.13) imply that the configuration $\psi^{(k)}(\theta)$ satisfies the restrictions defining the characteristic functions $\chi_{k}$ and (2.2) with the additional factor $\frac{1}{2}$ on the right-hand sides. The remaining conditions defining $\chi^{(k)}$ are formulated in terms of the fluctuation variables $\psi^{\prime}$, so by definition the configuration $\psi^{(k)}(\theta)$ is in a center of the corresponding domain. Thus this configuration is inside the domain of integration in (2.1). Let us notice in connection with the definition (2.31) that the factor $\frac{1}{2}$ is to some extent arbitrary, any number smaller than 1 , but not too small, could be used.

Making the change of variables $\psi=\psi^{(k)}+\psi^{\prime}$ we write the integral (2.1) in terms of the fluctuation variables $\psi^{\prime}$. We have studied the expansion in $\psi^{\prime}$ for the main term of the effective action. Consider now the remaining terms. They have the representations $(\mathrm{H} .2),(\mathrm{H} .7)$, and the $j^{\text {th }}$ terms in those representations depend on $\psi_{k}^{(j)}(\psi)$. The expansions

$$
\psi=\psi^{(k)}(\theta)+\psi^{\prime}, \quad \phi_{k}(\psi)=\phi_{k+1}(\theta)+\delta \phi_{k}\left(\psi^{\prime}\right), \quad \alpha_{k}(\psi)=\alpha_{k+1}(\theta)+\delta \alpha_{k}\left(\psi^{\prime}\right)
$$

and the formula (4.21) in [2] imply corresponding expansions of $\psi_{k}^{(j)}$, i.e. we have

$$
\psi_{k}^{(j)}(\psi)=\psi_{k+1}^{(j)}(\theta)+\delta \psi_{k}^{(j)}\left(\psi^{\prime}\right),
$$

where the last function on the right-hand side is given by

$$
\delta \psi_{k}^{(j)}\left(\psi^{\prime}\right)=Q_{j} \delta \phi_{k}\left(\psi^{\prime}\right)+\frac{a_{k}}{a_{j}}\left(L^{j} \eta\right)^{2} Q_{k-j}^{*}\left(\psi^{\prime}-Q_{k} \delta \phi_{k}\left(\psi^{\prime}\right)\right)
$$

The expansion (2.33) determines expansions of $\mathscr{E}^{(j)}\left(\psi_{k}^{(j)}\right)$ and $\mathscr{F}^{(j)}\left(\psi_{k}^{(j)}\right)$, for example

$$
\mathscr{E}^{(j)}\left(\psi_{k}^{(j)}(\psi)\right)=\mathscr{E}^{(j)}\left(\psi_{k+1}^{(j)}(\theta)\right)+\delta \mathscr{E}^{(j)}\left(\delta \psi_{k}^{(j)}\left(\psi^{\prime}\right)\right) .
$$

Denote the sum over $j$ of the last terms above by $\delta \mathscr{E}_{k}\left(\psi^{\prime}\right)$. Similar formulas hold for $\mathscr{F}^{(j)}$ and $\delta \mathscr{F}_{k}$. All these expansion formulas yield the following representation of the renormalization transformation $(2.1)$ :

$$
\begin{aligned}
\chi_{k+1, u}\left(T^{(k)} \chi^{(k)} \rho_{k}\right)(\theta) & \\
= & \chi_{k+1, u} \exp \left[-\beta_{k} A_{k+1}^{\eta}\left(\theta, \phi_{k+1}\right)+\mathscr{E}_{k}\left(\psi^{(k)}(\theta)\right)-E_{k}^{\prime}+\mathscr{F}_{k}\left(\psi^{(k)}(\theta), g\right)\right] \\
& \cdot \int d \psi^{\prime} \chi^{(k)} \chi_{k} \exp \left[-\beta_{k} \frac{1}{2}\left(a L^{-2}\left\|Q \psi^{\prime}\right\|_{L}^{2}+\left\langle\psi^{\prime}, \Delta^{(k)} \psi^{\prime}\right\rangle\right)\right. \\
& \left.-\beta_{k} V^{(k)}\left(\psi^{\prime}\right)+\delta \mathscr{E}_{k}\left(\psi^{\prime}\right)+\delta \mathscr{F}_{k}\left(\psi^{\prime}, g\right)\right]
\end{aligned}
$$


The Gaussian part of the above integral has the covariance $C^{(k)}$, i.e.

$$
C^{(k)}=\left(a L^{-2} Q^{*} Q+\Delta^{(k)}\right)^{-1} .
$$

It is a positive operator and its square root $C^{(k) \frac{1}{2}}$ is a positive operator also. We make a next change of variables in the fluctuation integral in (2.36), we take

$$
\psi^{\prime}=\beta_{k}^{-\frac{1}{2}} C^{(k) \frac{1}{2}} \psi
$$

The variables $\psi$ are the final fluctuation variables in terms of which we write the integral. The reason for introducing them is that the quadratic form in the integral becomes the purely local quadratic form $\frac{1}{2}\|\psi\|^{2}$, which simplifies a construction of localization expansions.

Consider the characteristic functions in the intergral in (2.36). We would like to define restrictions on the fluctuation variables $\psi$ in such a way, that the functions $\chi_{k}$ and (2.2) are equal to 1 on the domain of integration, taking into account the characteristic function $\chi_{k+1, u}$. This is satisfied if the following bounds hold:

$$
\left|\beta_{k}^{-\frac{1}{2}} C^{(k) \frac{1}{2}} \psi\right|,\left|\delta \phi_{k}\right|,\left|\partial^{\eta} \delta \phi_{k}\right|,\left|\Delta^{\eta} \delta \phi_{k}\right|,\left|\delta \alpha_{k}\right|<\frac{1}{3} \beta_{k}^{-\frac{1}{2}} p\left(\beta_{k}\right)
$$

where we have put $\beta_{k}^{-\frac{1}{2}} C^{(k) \frac{1}{2}} \psi$ in the argument of the functions $\delta \phi_{k}, \delta \alpha_{k}$, and we have used the formula (2.13), the restrictions in (2.31), and the bound $v_{k} \leqq 1$. The operator $C^{(k) \frac{1}{2}}$ is bounded, e.g. by a constant $B_{5}$. Then by the inequality (2.18) all the expressions in (2.39) can be bounded by $K_{2} B_{5} \beta_{k}^{-\frac{1}{2}}|\psi|$, so it is enough to assume that $K_{2} B_{5}|\psi|<\frac{1}{3} p\left(\beta_{k}\right)$. Let us introduce the remaining characteristic function by the formula

$$
\chi\left(\left\{|\psi|<p_{1}\left(\beta_{k}\right)\right\}\right), \quad p_{1}\left(\beta_{k}\right)=A_{1}\left(\log \beta_{k}\right)^{p_{1}},
$$

where

$$
\frac{p_{1}\left(\beta_{k}\right)}{p\left(\beta_{k}\right)}=\frac{A_{1}}{A_{0}}\left(\log \beta_{k}\right)^{p_{1}-p_{0}} \leqq\left(3 B_{5} K_{2}\right)^{-1},
$$

and $p_{1}$ is an even integer greater than 2 . With these assumptions the above function is the only characteristic function left under the integral in (2.36), and we may identify it with $\chi^{(k)}$. Notice that this function is invariant with respect to orthogonal transformations $R \in O(N)$ of the variables $\psi$, and with respect to Euclidean transformations $r$ mapping the lattice $T_{1}^{(k)}$ onto itself.

The change of variables (2.38) and the above statements on the characteristic functions yield the following equality:

$$
\begin{aligned}
& \chi_{k+1, u}\left(T^{(k)} \chi^{(k)} \rho_{k}\right)(\theta)=\chi_{k+1, u} \exp \left[-\beta_{k} A_{k+1}^{\eta}\left(\theta, \phi_{k+1}\right)+\mathscr{E}_{k}\left(\psi^{(k)}(\theta)\right)-E_{k}^{\prime \prime}\left|T_{1}^{(k)}\right|\right. \\
& \left.+\mathscr{F}_{k}\left(\psi^{(k)}(\theta), g\right)+\frac{1}{2} \log \operatorname{det} C^{(k)}\right] \cdot \int d \psi \chi^{(k)} \exp \left[-\frac{1}{2}\|\psi\|^{2}-\beta_{k} V^{(k)}\left(\beta_{k}^{-\frac{1}{2}} C^{(k) \frac{1}{2}} \psi\right)\right. \\
& \left.+\delta \mathscr{E}_{k}\left(\beta_{k}^{-\frac{1}{2}} C^{(k) \frac{1}{2}} \psi\right)+\delta \mathscr{F}_{k}\left(\beta_{k}^{-\frac{1}{2}} C^{(k) \frac{1}{2}} \psi, g\right)\right], \text { where } E_{k}^{\prime \prime}=E_{k}^{\prime}+\frac{1}{2} N \log \beta_{k} \cdot(2.41)
\end{aligned}
$$


The integral on the right-hand side above is the basic fluctuation integral. Its derivation has been done in the case $k \geqq 1$. The case $k=0$, which is connected with the first renormalization transformation, is slightly different, as we have remarked already in the first section. The difference is connected with the fact that the coefficient $\lambda$ may be arbitrarily large, in fact we may have $\lambda \rightarrow+\infty$ and we would like to have a procedure which is uniform in $\lambda$. Of course $\lambda_{k}$ may be arbitrarily large also, or $\lambda_{k} \rightarrow+\infty$, but the dependence on $\lambda_{k}$ in (2.41) is taken care of by the minimal configurations $\phi_{k+1}\left(\theta ; h, a_{k+1} L^{-2}, \lambda_{k}, v_{k}\right), \alpha_{k+1}\left(\theta ; h, a_{k+1} L^{-2}, \lambda_{k}, v_{k}\right)$, and all the expressions in (2.41) have properties and bounds uniform in $\lambda_{k}$. This would not be true in the first renormalization transformation; we have to perform some additional operations, and the whole procedure is slightly different, so we have to discuss it separately.

The first transformation applied to the initial density $\rho_{0}$ is defined by the integral

$$
\begin{aligned}
\left(T^{(0)} \chi^{(0)} \rho_{0}\right)(\psi)= & \int d \phi \chi_{0} \chi^{(0)} \exp \left[-\beta_{0}\left\{\frac{1}{2} a L^{-2}\|\psi-Q \phi\|_{L}^{2}+\frac{1}{2}\|\partial \phi\|^{2}\right.\right. \\
& \left.\left.+\frac{\lambda_{0}}{8}\left\||\phi|^{2}-1\right\|^{2}+\frac{v_{0}}{2}\|\phi-h\|^{2}\right\}+\langle g, \phi\rangle-E_{0}^{\prime}\left|T_{1}\right|\right],
\end{aligned}
$$

where the characteristic function $\chi_{0}$ is defined by the restrictions

$$
\begin{gathered}
\chi_{0}=\chi\left(\left\{|\psi-Q \phi|<2 \beta_{0}^{-\frac{1}{2}} p\left(\beta_{0}\right) \text { on } T^{\prime},|\partial \phi|<\beta_{0}^{-\frac{1}{2}} p\left(\beta_{0}\right),\right.\right. \\
\left.\left.\left.|| \phi\right|^{2}-1\left|<\lambda_{0}^{-\frac{1}{2}} \beta_{0}^{-\frac{1}{2}} p\left(\beta_{0}\right),\right| \phi-h \mid<v_{0}^{-\frac{1}{2}} \beta_{0}^{-\frac{1}{2}} p\left(\beta_{0}\right) \text { on } T\right\}\right), \\
\left.|| h\right|^{2}-1 \mid<v_{0}^{-1} \beta_{0}^{-1} p^{2}\left(\beta_{0}\right), \quad p\left(\beta_{0}\right)=A_{0}\left(\log \beta_{0}\right)^{p_{0}} .
\end{gathered}
$$

We expand the action in (2.42) around the minimal configuration $\phi_{1}$ taking $\phi=$ $\phi_{1}+\phi^{\prime}$. We have the equality

$$
\begin{gathered}
A_{1}\left(\psi, \phi ; h, a L^{-2}, \lambda_{0}, v_{0}\right)=A_{1}\left(\psi, \phi_{1} ; h, a L^{-2}, \lambda_{0}, v_{0}\right)+\left\{\frac{1}{2} a L^{-2}\left\|Q \phi^{\prime}\right\|^{2}\right. \\
\left.+\frac{1}{2}\left\|\partial \phi^{\prime}\right\|^{2}+\frac{1}{2}\left\langle\phi^{\prime},\left(v_{0}+\alpha_{1}\right) \phi^{\prime}\right\rangle+\frac{\lambda_{0}}{8}\left\|2 \phi_{1} \cdot \phi^{\prime}+\left|\phi^{\prime}\right|^{2}\right\|^{2}\right\},
\end{gathered}
$$

and we obtain an integral in $\phi^{\prime}$, for which the effective action has good positivity properties. To analyze this integral uniformly in $\lambda_{0}$ we make a change of variables. We decompose $\phi^{\prime}$ into two orthogonal components $\phi^{\prime}=\chi\left(\phi_{1}\right)_{0}+\phi^{\prime \prime}$, where $\left(\phi_{1}\right)_{0}=\frac{\phi_{1}}{\left|\phi_{1}\right|}, \chi=\left(\phi_{1}\right)_{0} \cdot \phi^{\prime},\left(\phi_{1}\right)_{0} \cdot \phi^{\prime \prime}=0$. Thus we have

$$
2 \phi_{1} \cdot \phi^{\prime}+\left|\phi^{\prime}\right|^{2}=2\left|\phi_{1}\right| \chi+\chi^{2}+\left|\phi^{\prime \prime}\right|^{2},
$$

and we can write the integral in (2.42) in terms of $\chi, \phi^{\prime \prime}$. Let us make some remarks about invariance properties of the expressions and variables above. The expression in the curly bracket in (2.44) is invariant with respect to simultaneous transformations of $\phi_{1}, \phi^{\prime}$ by elements of $O(N)$, so $\chi$ is invariant and the expression is invariant with respect to the transformations of $\phi, \phi^{\prime \prime}$. The characteristic function $\chi^{(0)}$, which will be defined below, restricts $\phi^{\prime}$ to a sufficiently small neighborhood of $0\left(\left|\phi^{\prime}\right|<O\left(\beta_{0}^{-\frac{1}{2}} p\left(\beta_{0}\right)\right)\right)$, hence $\chi, \phi^{\prime \prime}$ are small also. We make a change of variables 
$\chi=\chi\left(\chi^{\prime}, \phi^{\prime \prime}\right)$ such that

$$
2\left|\phi_{1}\right| \chi+\chi^{2}+\left|\phi^{\prime \prime}\right|^{2}=2\left|\phi_{1}\right| \chi^{\prime}
$$

This equation has a unique solution

$$
\chi=\sqrt{\left|\phi_{1}\right|^{2}+2\left|\phi_{1}\right| \chi^{\prime}-\left|\phi^{\prime \prime}\right|^{2}}-\left|\phi_{1}\right|=\chi^{\prime}+h_{2}\left(\chi^{\prime}, \phi^{\prime \prime}\right),
$$

where $h_{2}$ is an analytic function with an expansion starting with second order terms. This change of variables transforms the integral in (2.42) into an integral in variables $\chi^{\prime}, \phi^{\prime \prime}$. Finally, we rescale these variables:

$$
\phi^{\prime \prime}=\beta_{0}^{-\frac{1}{2}} \phi, \quad \chi^{\prime}=\left(\lambda_{0} \beta_{0}\right)^{-\frac{1}{2}} \chi
$$

The above transformations give a fluctuation integral in which the integration variables $\phi, \chi$ are restricted by the condition $\left(\phi_{1}\right)_{0} \cdot \phi=0$ and by the characteristic functions $\chi_{0}, \chi^{(0)}$. We remove $\chi_{0}$ from the integral by introducing new restrictions on $\psi$ by the characteristic function

$$
\begin{aligned}
\chi_{1, u}=\chi( & \left\{\left|\psi-Q \phi_{1}\right|<\frac{3}{2} \beta_{0}^{-\frac{1}{2}} p\left(\beta_{0}\right) \text { on } T_{L}^{(1)},\left|\partial \phi_{1}\right|<\frac{1}{2} \beta_{0}^{-\frac{1}{2}} p\left(\beta_{0}\right),\left|\Delta \phi_{1}\right|\right. \\
& \left.\left.<\frac{1}{2} \beta_{0}^{-\frac{1}{2}} p\left(\beta_{0}\right),\left|\alpha_{1}\right|<\frac{1}{2} \beta_{0}^{-\frac{1}{2}} p\left(\beta_{0}\right),\left|\phi_{1}-h\right|<\frac{1}{2} v_{0}^{-\frac{1}{2}} \beta_{0}^{-\frac{1}{2}} p\left(\beta_{0}\right) \text { on } T_{1}\right\}\right) .
\end{aligned}
$$

We assume sufficiently strong restrictions on $\phi, \chi$, so that $\chi_{1, u} \chi_{0} \chi^{(0)}=\chi_{1, u} \chi^{(0)}$. Using this equality we can write the following formula for the renormalization transformation (2.42):

$$
\begin{aligned}
& \chi_{1, u}\left(T^{(0)} \chi^{(0)} \rho_{0}\right)(\psi)=\chi_{1, u} \exp \left[-\beta_{0} A_{1}\left(\psi, \phi_{1} ; h, a L^{-2}, \lambda_{0}, v_{0}\right)+\left\langle g, \phi_{1}\right\rangle-E_{0}^{\prime \prime}\left|T_{1}\right|\right] \\
& \cdot \int d \phi d \chi \delta\left(\left(\phi_{1}\right)_{0} \cdot \phi\right) \chi^{(0)} \exp \left[-\frac{1}{2}\left\langle\phi+\lambda_{0}^{-\frac{1}{2}}\left(\phi_{1}\right)_{0} \chi,\left(-\Delta+a L^{-2} Q^{*} Q+v_{0}+\alpha_{1}\right)\right.\right. \\
& \left.\cdot\left(\phi+\lambda_{0}^{-\frac{1}{2}}\left(\phi_{1}\right)_{0} \chi\right)\right\rangle-\frac{1}{2}\left\langle\chi,\left|\phi_{1}\right|^{2} \chi\right\rangle-\beta_{0} V^{(0)}\left(\beta_{0}^{-\frac{1}{2}} \phi,\left(\beta_{0} \lambda_{0}\right)^{-\frac{1}{2}} \chi\right) \\
& \left.\quad+\left\langle g, \beta_{0}^{-\frac{1}{2}} \phi+\left(\phi_{1}\right)_{0}\left(\left(\beta_{0} \lambda_{0}\right)^{-\frac{1}{2}} \chi+h_{2}\right)\right\rangle\right]
\end{aligned}
$$

where we have separated terms quadratic in the fluctuation variables from terms of higher order, or small. These are gathered together in the function $V^{(0)}$, and we have

$$
\begin{aligned}
& V^{(0)}\left(\beta_{0}^{-\frac{1}{2}} \phi,\left(\beta_{0} \lambda_{0}\right)^{-\frac{1}{2}} \chi\right) \\
& =\beta_{0}^{-\frac{1}{2}}\left\langle\phi+\lambda_{0}^{-\frac{1}{2}}\left(\phi_{1}\right)_{0} \chi, G_{1}^{-1}\left(\alpha_{1}\right)\left(\phi_{1}\right)_{0} h_{2}\right\rangle+\frac{1}{2}\left\langle\left(\phi_{1}\right)_{0} h_{2}, G_{1}^{-1}\left(\alpha_{1}\right)\left(\phi_{1}\right)_{0} h_{2}\right\rangle \\
& \quad+\beta_{0}^{-1} \frac{1}{2} \sum_{x \in T_{1}} \log \left(1+2\left(\beta_{0} \lambda_{0}\right)^{-\frac{1}{2}} \frac{\chi(x)}{\left|\phi_{1}(x)\right|}-\beta_{0}^{-1} \frac{|\phi(x)|^{2}}{\left|\phi_{1}(x)\right|^{2}}\right)
\end{aligned}
$$


where $h_{2}=h_{2}\left(\left(\beta_{0} \lambda_{0}\right)^{-\frac{1}{2}} \chi, \beta_{0}^{-\frac{1}{2}} \phi\right)$ is given by (2.45), and

$$
G_{1}^{-1}\left(\alpha_{1}\right)=-\Delta+a L_{1}^{-2} Q^{*} Q+v_{0}+\alpha_{1} .
$$

Consider the function $V^{(0)}$. It is an almost local function of the fluctuation variables, more precisely it is a sum of terms involving the variables either at one site, or at two nearest neighbor sites, or at sites of one block. Each term has also the same type of the almost local dependence on $\phi_{1}$. It is analytic in $\phi_{1}$ and small. The function $h_{2}$ is at least of second order in $\chi, \phi$, so it is $O\left(\beta_{0}^{-1} p_{1}^{2}\left(\beta_{0}\right)\right)$, and the formula (2.47) implies that $\beta_{0} V^{(0)}=O\left(\beta_{0}^{-\frac{1}{2}} p_{1}^{3}\left(\beta_{0}\right)\right)\left|T_{1}\right|$.

Consider the integral in (2.46). From the above it follows that the integrand is a small and local perturbation of a Gaussian measure in the variables $\phi, \chi, \phi$ in the subspace defined by the condition $\left(\phi_{1}\right)_{0} \cdot \phi=0$. We make a change of variables introducing a new variable $\phi^{\prime}$ by the equality $\phi^{\prime}=\phi+\left(\phi_{1}\right)_{0} \chi$. This new variable considered at any point of the lattice $T_{1}$ has values in the whole space $\mathbb{R}^{N}$, unrestricted by any conditions. This change of variables is given by an orthogonal transformation between the corresponding spaces, so the Jacobian is equal to 1 . The quadratic form in (2.46) can be written in the new variable $\phi^{\prime}$ as

$$
\begin{aligned}
& \left\langle\phi^{\prime},\left[I-\left(1-\lambda_{0}^{-\frac{1}{2}}\right)\left(\phi_{1}\right)_{0} \otimes\left(\phi_{1}\right)_{0}\right] G_{1}^{-1}\left(\alpha_{1}\right)\left[I-\left(1-\lambda_{0}^{-\frac{1}{2}}\right)\left(\phi_{1}\right)_{0} \otimes\left(\phi_{1}\right)_{0}\right] \phi^{\prime}\right\rangle \\
& \quad+\left\langle\phi^{\prime},\left(1+2 \frac{\alpha_{1}}{\lambda_{0}}\right)\left(\phi_{1}\right)_{0} \otimes\left(\phi_{1}\right)_{0} \phi^{\prime}\right\rangle .
\end{aligned}
$$

The operator of this form is bounded and short-ranged. The operator $G_{1}^{-1}\left(\alpha_{1}\right)$ is bounded from below by an absolute positive constant $\gamma_{0}$ (see [3] for a proof), and $\alpha_{1}$ is bounded by a small constant $c_{1}$. Thus the quadratic form (2.49) is bounded from below by

$$
\gamma_{0}\left\langle\phi^{\prime},\left[I-\left(\phi_{1}\right)_{0} \otimes\left(\phi_{1}\right)_{0}\right] \phi^{\prime}\right\rangle+\left(1+\frac{1-2 c_{0}}{\lambda_{0}}\right)\left\langle\phi^{\prime},\left(\phi_{1}\right)_{0} \otimes\left(\phi_{1}\right)_{0} \phi^{\prime}\right\rangle \geqq \gamma_{0}\left\langle\phi^{\prime}, \phi^{\prime}\right\rangle,
$$

assuming that $c_{1}<\frac{1}{2}$ and $\gamma_{0} \leqq 1$. From the above properties it follows that the operator of the quadratic form (2.49) satisfies the assumptions of the theorem on unit lattice operators in [3], so its inverse has an exponentially decaying kernel and a localization expansion (see (5.17) in [3]). Denote the inverse by $C^{(0)}$. The square root of this operator has the same properties. We make the next change of variables $\phi^{\prime}=C^{(0) \frac{1}{2}} \phi$, which yields the following formula for the first renormalization transformation:

$$
\begin{aligned}
& \chi_{1, u}\left(T^{(0)} \chi^{(0)} \rho_{0}\right)(\psi) \\
& =\chi_{1, u} \exp \left[-\beta_{0} A_{1}\left(\psi, \phi_{1} ; h, a L^{-2}, \lambda_{0}, v_{0}\right)+\frac{1}{2} \log \operatorname{det} C^{(0)}\right. \\
& \left.\quad-E_{0}^{\prime \prime}\left|T_{1}\right|+\left\langle g, \phi_{1}\right\rangle\right] \int d \phi \chi^{(0)} \exp \left[-\frac{1}{2}\|\phi\|^{2}-\beta_{0} V^{(0)}\left(\beta_{0}^{-\frac{1}{2}} C^{(0) \frac{1}{2}} \phi\right)\right. \\
& \left.\quad+\left\langle g, \beta_{0}^{-\frac{1}{2}}\left[I-\left(1-\lambda_{0}^{-\frac{1}{2}}\right)\left(\phi_{1}\right)_{0} \otimes\left(\phi_{1}\right)_{0}\right] C^{(0) \frac{1}{2}} \phi+\left(\phi_{1}\right)_{0} h_{2}\right\rangle\right] .
\end{aligned}
$$


The functions $V^{(0)}$ and $h_{2}$ above are obtained from the corresponding functions in $(2.46)$ by substituting $\left(I-\left(\phi_{1}\right)_{0} \otimes\left(\phi_{1}\right)_{0}\right) C^{(0) \frac{1}{2}} \phi,\left(\phi_{1}\right)_{0} \cdot C^{(0) \frac{1}{2}} \phi$ in place of $\phi, \chi$. We define the characteristic function $\chi^{(0)}$ by $(2.40)$ with $k=0$. The fluctuation integral in (2.50) has the same form and properties as the integral in (2.41). The underintegral expressions have bounds uniform in the whole range of $\lambda_{0}$, in fact they are analytic functions of $\lambda_{0}^{-\frac{1}{2}}$. The integral in (2.50) is now simpler than the integral in (2.41), so we continue our analysis of the last one.

The integral in (2.41) is the basic fluctuation integral which we will analyze now, and also in the next section. At first let us describe some of its basic properties. It is obviously convergent and defines a function of $\theta, h, g$ through various functions $\psi_{k+1}^{(j)}, \mathscr{E}^{(j)}, \mathscr{F}^{(j)}$. By the previously described symmetry properties it is invariant with respect to Euclidean transformations of $\theta, h, g$, which map the lattice $T^{(k+1)}$ onto itself, and with respect to orthogonal transformations $R \in O(N)$ of $\theta, h, g$. This integral determines new contributions to the effective action and the generating functional. Let us write explicitly formulas for these contributions. Define a two-parameter family of measures

$$
\begin{aligned}
d \mu_{s, t}(\psi)= & \left(Z_{s, t}^{(k)}\right)^{-1} d \psi \chi^{(k)} \exp \left[-\frac{1}{2}\|\psi\|^{2}-\beta_{k} V^{(k)}\left(\beta_{k}^{-\frac{1}{2}} C^{(k) \frac{1}{2}} s \psi\right)\right. \\
& \left.+\delta \mathscr{E}_{k}\left(\beta_{k}^{-\frac{1}{2}} C^{(k) \frac{1}{2}} s \psi\right)+\delta \mathscr{F}_{k}\left(\beta_{k}^{-\frac{1}{2}} C^{(k) \frac{1}{2}} t \psi, g\right)\right],
\end{aligned}
$$

where $Z_{s, t}^{(k)}$ is a normalization factor, or the integral over $\psi$ of the expression after $d \psi$ above. The new contribution to the generating functional is given by

$$
\begin{aligned}
\mathscr{F}_{0}^{(k+1)}(g) & =\left\langle g, \beta_{k}^{-\frac{1}{2}} \mathscr{M}_{0}^{(k+1)}(g)\right\rangle_{1}=\int_{0}^{1} d t \int d \mu_{1, t}(\psi) \frac{d}{d t} \delta \mathscr{F}_{k}\left(\beta_{k}^{-\frac{1}{2}} C^{(k) \frac{1}{2}} t \psi\right) \\
& =\beta_{k}^{-\frac{1}{2}} \int_{0}^{1} d t \int d \mu_{1, t}(\psi)\left\langle\left(\frac{\partial}{\partial \psi^{\prime}} \delta \mathscr{F}_{k}\right)\left(\beta_{k}^{-\frac{1}{2}} C^{(k) \frac{1}{2}} t \psi\right), C^{(k) \frac{1}{2}} \psi\right\rangle,
\end{aligned}
$$

or

$$
\begin{aligned}
& \mathscr{M}_{0}^{(k+1)}(x ; g)=\int_{0}^{1} d t \int d \mu_{1, t}(\psi)\left\langle\left(\frac{\partial}{\partial \psi^{\prime}} \delta \phi_{k}\right)\left(x ; \beta_{k}^{-\frac{1}{2}} C^{(k) \frac{1}{2}} t \psi\right), C^{(k) \frac{1}{2}} \psi\right\rangle \\
& +\sum_{j=1}^{k} \int_{0}^{1} d t \int d \mu_{1, t}(\psi)\left\langle\left(\frac{\partial}{\partial \psi^{\prime}} \delta \mathscr{M}^{(j)}\right)\left(x ; \delta \psi_{k}^{(j)}\left(\beta_{k}^{-\frac{1}{2}} C^{(k) \frac{1}{2}} t \psi\right), g\right), C^{(k) \frac{1}{2}} \psi\right\rangle .
\end{aligned}
$$

The new contribution to the effective action is given by

$$
\mathscr{E}_{0}^{(k+1)}=\frac{1}{2} \log \operatorname{det} C^{(k)}+\mathscr{E}_{1}^{(k+1)}
$$


where

$$
\begin{aligned}
\mathscr{E}_{1}^{(k+1)}= & \int_{0}^{1} d s \int d \mu_{s, 0}(\psi)\left(-\beta_{k}^{\frac{1}{2}}\left\langle\left(\frac{\partial}{\partial \psi^{\prime}} V^{(k)}\right)\left(\beta_{k}^{-\frac{1}{2}} C^{(k) \frac{1}{2}} s \psi\right), C^{(k) \frac{1}{2}} \psi\right\rangle\right. \\
& \left.+\beta_{k}^{-\frac{1}{2}}\left\langle\left(\frac{\partial}{\partial \psi^{\prime}} \delta \mathscr{E}_{k}\right)\left(\beta_{k}^{-\frac{1}{2}} C^{(k) \frac{1}{2}} s \psi\right), C^{(k) \frac{1}{2}} \psi\right\rangle\right) .
\end{aligned}
$$

The above expression can be written as a sum of localized terms

$$
\mathscr{E}_{1}^{(k+1)}=\sum_{z \in T_{L}^{(k+1)}} \mathscr{E}_{1}^{(k+1)}(z)
$$

which are given by the following formula:

$$
\begin{aligned}
\mathscr{E}_{1}^{(k+1)}(z)= & -\beta_{k}^{\frac{1}{2}} \sum_{y \in B(z)} \int_{0}^{1} d s \int d \mu_{s, 0}(\psi)\left(\frac{\partial}{\partial \psi^{\prime}(y)} V^{(k)}\right)\left(\beta_{k}^{-\frac{1}{2}} C^{(k) \frac{1}{2}} s \psi\right) \\
& \cdot\left(C^{(k) \frac{1}{2}} \psi\right)(y)+\beta_{k}^{-\frac{1}{2}} \sum_{y \in B(z)} \int_{0}^{1} d s \int d \mu_{s, 0}(\psi)\left(\frac{\partial}{\partial \psi^{\prime}(y)} \delta \mathscr{E}_{k}\right) \\
& \times\left(\beta_{k}^{-\frac{1}{2}} C^{(k) \frac{1}{2}} s \psi\right) \cdot\left(C^{(k) \frac{1}{2}} \psi\right)(y) .
\end{aligned}
$$

The two formulas $(2.57),(2.53)$ are the fundamental formulas for the future discussion of localization expansions and bounds. We have to write also $\frac{1}{2} \log \operatorname{det} C^{(k)}$ as a sum of localized terms. We have

$$
\begin{aligned}
\frac{1}{2} \log \operatorname{det} C^{(k)} & =-\frac{1}{2} \log \operatorname{det}\left(a L^{-2} Q^{*} Q+\Delta^{(k)}\right) \\
& =-\frac{1}{2} \operatorname{Tr} \log \left(a L^{-2} Q^{*} Q+\Delta^{(k)}\right) \\
& =-\frac{1}{2} \sum_{y \in T_{1}^{(k)}} \operatorname{tr}\left(\log \left(a L^{-2} Q^{*} Q+\Delta^{(k)}\right)\right)(y, y),
\end{aligned}
$$

where the symbol "tr" denotes algebraic trace of $N \times N$-matrix.

This allows us to write $\mathscr{E}_{0}^{(k+1)}$ as a sum of localized terms

$$
\mathscr{E}_{0}^{(k+1)}=\sum_{z \in T_{L}^{(k+1)}} \mathscr{E}_{0}^{(k+1)}(z)
$$

where

$$
\mathscr{E}_{0}^{(k+1)}(z)=-\frac{1}{2} \sum_{y \in B(z)} \operatorname{tr}\left(\log \left(a L^{-2} Q^{*} Q+\Delta^{(k)}\right)\right)(y, y)+\mathscr{E}_{1}^{(k+1)}(z) .
$$

From the previously discussed symmetry properties it follows that

$$
\mathscr{E}_{0}^{(k+1)}(r z ; r \theta, h)=\mathscr{E}_{0}^{(k+1)}(z ; \theta, h)
$$


for Euclidean transformations $r$ mapping $T^{(k+1)}$ onto itself, and

$$
\mathscr{E}_{0}^{(k+1)}(z ; R \theta, R h)=\mathscr{E}_{0}^{(k+1)}(z ; \theta, h)
$$

for orthogonal transformations $R \in O(N)$. Similar properties hold for the new contribution to the generating functional, i.e. for (2.52), or (2.53).

The equality (2.41) and the definitions (2.51)-(2.60) yield the equality

$$
\begin{aligned}
\chi_{k+1, u}\left(T^{(k)} \chi^{(k)} \rho_{k}\right)(\theta)= & \chi_{k+1, u} \exp \left[-\beta_{k} A_{k+1}^{\eta}\left(\theta, \phi_{k+1}\right)+\mathscr{E}_{k}\left(\psi^{(k)}(\theta)\right)+\mathscr{E}_{0}^{(k+1)}(\theta)\right. \\
& \left.-E_{k}^{\prime \prime \prime}\left|T_{1}^{(k)}\right|+\mathscr{F}_{k}\left(\psi^{(k)}(\theta), g\right)+\mathscr{F}_{0}^{(k+1)}(\theta, g)\right],
\end{aligned}
$$

where

$$
E_{k}^{\prime \prime \prime}=E_{k}^{\prime \prime}-\log \int_{\mathbb{R}^{N}} d \psi \chi\left(\left\{|\psi|<p_{1}\left(\beta_{k}\right)\right\}\right) e^{-\frac{1}{2}|\psi|^{2}} .
$$

The density on the right-hand side has already some basic features of densities in a space (1.4) with $k+1$ instead of $k$. The new contributions are defined at the moment on the domain of the characteristic function $\chi_{k+1, u}$. We would like to show that they have localization expansions satisfying (H.4) and the corresponding properties in (H.7). These imply all the analyticity properties in the inductive hypotheses, as we have noticed already before. A construction of the localization expansion will be given in a later paper, now we formulate the results only. A first result concerns the new terms $\mathscr{E}_{0}^{(k+1)}(z)$ of the effective action, and is described in the following proposition.

Proposition 2.1. If $\beta$ or $B$ are large enough, then the functions $\mathscr{E}_{0}^{(k+1)}$ defined by the formulas (2.60), (2.57) have localization expansions satisfying all the conditions of the inductive hypothesis (H.4) for $j=k+1$, but the bounds hold with $E_{0}$ replaced by $N L^{d} B_{4}+1$, and $\kappa$ replaced by $2 \kappa-3-6 \kappa_{0}$.

Let us recall that the "absolute" constant $B_{4}$ was defined in Sect. 3 of [3] in connection with the localization of the functions of the operator $a L^{-2} Q^{*} Q+\Delta^{(k)}$, where $\Delta^{(k)}$ is defined by (2.23). Obviously we assume here that $\kappa-3-6 \kappa_{0} \geqq 0$. It follows from the above proposition that we can take $E_{0}$ as any number $\geqq N L^{d} B_{4}+1$. This is not a final restriction on $E_{0}$ because there will be other contributions to the effective action introduced in the next sections. We have a similar result for the new contribution to the generating functional.

Proposition 2.2. For $\beta$ or $B$ large enough the functions $\mathscr{F}_{0}^{(k+1)}(x ; g)$ defined by (2.52),(2.53) satisfy all the conditions of the inductive hypothesis (H.7) for $j=k+1$, but the bounds hold with $c_{9}$ replaced by const. $\beta^{-\frac{5}{8}+\alpha_{1}} \leqq \frac{1}{2} \beta^{-\frac{1}{4}}$, and $\kappa$ replaced by $2 \kappa-4-6 \kappa_{0}$.

From this proposition we obtain that we can take $c_{9}$ as any number $\geqq \frac{1}{2} \beta^{-\frac{1}{4}}$. Again it is not a final restriction on $c_{9}$, we will have other contributions to the generating functional. We assume that $\kappa-4-6 \kappa_{0} \geqq 0$, and the exponential factors in the bounds for the new contributions $\mathscr{E}_{0}^{(k+1)}, \mathscr{F}_{0}^{(k+1)}(g)$ can be taken exactly the same as in the hypotheses (H.4), (H.7).

The above two propositions will be proved in a more general form in one of the following papers. The generalization is connected with restricting the effective action and the fluctuation integral to "a small field" subregion of the whole lattice, and also with some other technical issues. 


\section{The Scaling Operation $S^{(k)}$}

A next operation is the scaling operation $S^{(k)}$. It is simply a rescaling of the lattice $T_{\eta}$ to $T_{L^{-1} \eta}$, and corresponding rescalings of the lattices $T_{L^{\prime} \eta}^{(j)}$ to the lattices $T_{L^{\prime-1}}^{(j)} \eta^{\text {. }}$ The spin configurations and the external fields are unchanged by the rescalings, or their values on the rescaled lattices are equal to values at corresponding points of the original lattices. Functions of the spin configurations and the external fields, like the effective action and the generating functional, do not change also, they are defined on new lattices, domains and configurations in the same way as on the original ones. An exception in this is the main action which is written explicitly in terms of the $L^{2}$-norms on relevant lattices. The rescaling implies the following equality:

$$
\beta_{k} A_{k+1}^{\eta}\left(\theta, \phi_{k+1} ; a_{k+1} L^{-2}, \lambda_{k}, v_{k}\right)=\beta_{k} L^{d-2} A_{k+1}\left(\theta, \phi_{k+1} ; a_{k+1, u}, \lambda_{k+1, u}, v_{k+1, u}\right),
$$

where the action and the configurations on the right-hand side are defined on the $L^{-1} \eta$-lattice, and $\lambda_{k+1, u}=\lambda_{k} L^{2}, v_{k+1, u}=v_{k} L^{2}$. We have also the scaling property (2.12) for the function $\phi_{k+1}$, so the rescaled function depends on $a_{k+1, u}, \lambda_{k+1, u}, v_{k+1, u}$. We write all these expressions with the subscript " $u$ " to indicate that they are rescaled but unrenormalized. A result of the two operations is written in the following way:

$$
\begin{gathered}
\chi_{k+1, u}\left(S^{(k)} T^{(k)} \chi^{(k)} \rho_{k}\right)(\theta)=\chi_{k+1, u} \exp \left[-\beta_{k+1, u} A_{k+1, u}\left(\theta, \phi_{k+1, u}\right)+\mathscr{E}_{k}\left(\psi_{u}^{(k)}(\theta)\right)\right. \\
\left.+\mathscr{E}_{0}^{(k+1)}(\theta)-E_{k}^{\prime \prime \prime} L^{d}\left|T_{1}^{(k+1)}\right|+\mathscr{F}_{k}\left(\psi_{u}^{(k)}(\theta), g\right)+\mathscr{F}_{0}^{(k+1)}(\theta, g)\right]
\end{gathered}
$$

where

$$
\beta_{k+1, u}=\beta_{k} L^{d-2}, A_{k+1, u}(\theta, \phi)=A_{k+1}\left(\theta, \phi ; a_{k+1, u}, \lambda_{k+1, u}, v_{k+1, u}\right) .
$$

The characteristic function is written also in terms of the rescaled expressions. The domain of this function is basically equal to the space $\Xi_{k+1, u}\left(L, \frac{1}{2} \beta_{k}^{-\frac{1}{2}} p\left(\beta_{k}\right)\right)$, except that in the last condition in (2.20) [1] the constant $L^{2} \frac{1}{2} \beta_{k}^{-\frac{1}{2}} p\left(\beta_{k}\right)$ is replaced by $\frac{3}{2} \beta_{k}^{-\frac{1}{2}} p\left(\beta_{k}\right)$.

Let us summarize properties of the density on the right-hand side of (3.2). The "old" terms $\mathscr{E}_{k}$ and $\mathscr{F}_{k}$ satisfy all the inductive hypothesis (H.2)-(H.5),(H.7) but now the $j^{\text {th }}$ terms depend on the unrenormalized function $\psi_{k+1, u}^{(j)}(\theta)$ instead of the function $\psi_{k}^{(j)}\left(\psi_{k}\right)$. The analyticity domains are also defined in terms of the unrenormalized functions $\phi_{j, u}$, and we denote them by $\Xi_{j, u}^{c}\left(1, \varepsilon_{j, u}\right)$, where $\varepsilon_{j, u}$ denotes the constant after $k$ steps, which differs by the term $\frac{1}{k^{2}} \alpha_{0} \xi^{\alpha}$ from the constant after $k+1$ steps. After a renormalization operation we will have to change accordingly the spaces and to prove proper inclusions. The "new" terms $\mathscr{E}_{0}^{(k+1)}, \mathscr{F}_{0}^{(k+1)}$ satisfy the inductive hypotheses (H.2)-(H.4), (H.7) only, but with the analyticity domain $\Xi_{k+1, u}^{c}\left(1, \varepsilon_{k+1}\right)$ defined also in terms of the unrenormalized function $\phi_{k+1, u}$, and with constants in the bounds in (H.4), (H.7) independent of $E_{0}$. These constants are described more precisely in Propositions 2.2,2.3.

Our basic goal now is to modify the effective action in (3.2) in such a way that it satisfies all the inductive hypotheses (H.1)-(H.7). This is achieved by a renormalization operation defined and discussed in the next two sections. 


\section{The Renormalization Operation}

In the small field region considered here this operation is reduced to rearrangements of the effective action done in such a way that they create new terms which are combined with the term $\mathscr{E}_{0}^{(k+1)}$ and yield an expression satisfying the renormalization conditions (H.5). They involve a rescaling of the variable $\theta$ and changes of the constants $\beta_{k+1, u}, a_{k+1, u}, \lambda_{k+1, u}, v_{k+1, u}$ defining the main action. Before we describe these operations let us explain why do we need the rescaling. It is connected with properties of the effective potential, and in particular with the second condition in (H.5). The effective potential for the whole effective action in (3.2) is defined as the value of this action on a constant configuration $\theta$, divided by the volume $\left|T_{1}^{(k+1)}\right|$ of the lattice. This gives the expression

$$
\mathscr{V}_{k+1}(\theta, h)=\beta_{k+1} V_{k+1}(\theta, h)-\sum_{j=1}^{k}\left(L^{j-1} \eta\right)^{-d} \mathscr{V}^{(j)}\left(\psi_{k+1}^{(j)}(\theta, h), h\right)-\mathscr{V}_{0}^{(k+1)}(\theta, h),
$$

where for simplicity we have dropped the subscript $u$. We can write it in terms of the corresponding functions of the expressions $U, V, W^{2}$ as in (3.45) [1]. We are interested now only in $\theta=u e_{1}, h=e_{1}$, so we obtain

$$
\begin{aligned}
f_{k+1}(u, 1,0)= & \beta_{k+1} v_{k+1}(u, 1,0) \\
& -\sum_{j=1}^{k}\left(L^{j-1} \eta\right)^{-d} f^{(j)}\left(\left|\psi_{k+1}^{(j)}\left(u e_{1}, e_{1}\right)\right|, 1,0\right)-f_{0}^{(k+1)}(u, 1,0),
\end{aligned}
$$

where we have used the fact that $\psi_{k+1}^{(j)}\left(u e_{1}, e_{1}\right)$ is proportional to $e_{1}$, and its value at $u=1$ is equal to $e_{1}$. These properties follow immediately from the variational equations determining the functions $\psi_{k+1}^{(j)}$. It follows also that $u=1$ is a critical point of all the functions on the right-hand side above, except the last one, and this is true for all admissible values of the constants $a_{k+1}, \lambda_{k+1}, v_{k+1}$, allowed by the existence theory for the variational problem. This means that no change of the constants can produce a new contribution to $f_{0}^{(k+1)}(u, 1,0)$ changing the derivative $\frac{\partial}{\partial u} f_{0}^{(k+1)}(1,1,0)$, so it cannot make the point $u=1$ a critical point of the function $f_{0}^{(k+1)}(u, 1,0)$. We have to change this function in some other way. One possible way is suggested by the following remark. The whole effective potential $f_{k+1}(u, 1,0)$ has a critical point which is close to 1 . This follows from the fact that the first term in the definition of $f_{k+1}$ is dominating, so we can write

$$
f_{k+1}(u, 1,0)=O\left(\frac{1}{2} \beta_{k+1}(u-1)^{2}\right)-f_{0}^{(k+1)}(u, 1,0),
$$

and the equation

$$
\frac{\partial}{\partial u} f_{k+1}(u, 1,0)=O\left(\beta_{k+1}(u-1)\right)-\frac{\partial}{\partial u} f_{0}^{(k+1)}(u, 1,0)=0
$$

has exactly one solution in a neighborhood of 1 . Denoting this solution by $z_{k+1}$ we have $z_{k+1}=1+O\left(\beta_{k+1}^{-1}\right)$. Now we make the change of variables $u=z_{k+1} u^{\prime}$. The function $f_{k+1}\left(z_{k+1} u^{\prime}, 1,0\right)$ of $u^{\prime}$ has a critical point at $u^{\prime}=1$. We can write this 
function as

$$
\begin{aligned}
f_{k+1}\left(z_{k+1} u^{\prime}, 1,0\right)= & \beta_{k+1} v_{k+1}\left(u^{\prime}, 1,0\right) \\
& -\sum_{j=1}^{k}\left(L^{j-1} \eta\right)^{-d} f^{(j)}\left(\left|\psi_{k+1}^{(j)}\left(u^{\prime} e_{1}, e_{1}\right)\right|, v_{j}, 0\right)-f^{(k+1)}\left(u^{\prime}, 1,0\right),
\end{aligned}
$$

where the definition of $f^{(k+1)}$ is obvious. This function has similar properties and bounds as $f_{0}^{(k+1)}$ because $z_{k+1}-1=O\left(\beta_{k+1}^{-1}\right)$, so the factor $\beta_{k+1}$ in the leading term is cancelled. Obviously by the above definition the point $u^{\prime}=1$ is a critical point of the function $f^{(k+1)}\left(u^{\prime}, 1,0\right)$, so the second condition in (H.5) is satisfied. The above discussion should explain the role of the scaling transformation and the definition of the scaling constant. The remaining conditions in (H.5) can be satisfied by proper changes of the coefficients in the main action in (3.1). We do not consider these conditions separately, but we treat them all at once, in one renormalization operation.

Let us define now the basic step of the renormalization operation. It consists of the scaling operation and the change of the coefficients given by the equations

$$
\begin{aligned}
& \theta=z_{k+1} \psi_{k+1}, \quad z_{k+1}=1+\zeta_{k+1}, \\
& \beta_{k+1, u}=\beta_{k+1}+b_{k+1}, \quad \beta_{k+1, u} a_{k+1, u}=\beta_{k+1} a_{k+1}+c_{k+1}, \\
& \frac{\beta_{k+1, u}}{\lambda_{k+1, u}}=\frac{\beta_{k+1}}{\lambda_{k+1}}+\frac{1}{\lambda_{k+1}} d_{k+1}, \quad \beta_{k+1, u} v_{k+1, u}=\beta_{k+1} v_{k+1}+v_{k+1} e_{k+1} .
\end{aligned}
$$

The constants $\zeta_{k+1}, b_{k+1}, c_{k+1}, d_{k+1}, e_{k+1}$ will be determined later by the renormalization conditions (H.5). If they are given, then the above equations determine uniquely the new coefficients $\beta_{k+1}, a_{k+1}, \lambda_{k+1}, v_{k+1}$. The above equations determine a decomposition of the main action in (3.2) into a sum of a new action and a remaining expression which is called a counterterm. This decomposition is complicated by the fact that the minimal configuration $\phi_{k+1, u}$ is changed also by the change of the coefficients, we have to expand it around the new minimal configuration $\phi_{k+1}$ determined by the new, renormalized coefficients. We study simultaneously the decomposition of the main action and the expansion of the minimal configuration using the variational problem (1.6)-(1.9) [2]. We have

$$
\begin{aligned}
& \beta_{k+1, u} A_{k+1, u}\left(\theta, \phi_{k+1, u}\right)=\beta_{k+1, u} \inf _{\phi} A_{k+1, u}(\theta, \phi)=\beta_{k+1, u} \inf _{\phi} \sup _{\alpha} J_{k+1, u}(\theta, \phi, \alpha) \\
& =\frac{1}{2}\left(\beta_{k+1} a_{k+1}+c_{k+1}\right)\left(z_{k+1}-1\right)^{2}\left\|\psi_{k+1}\right\|^{2}+\inf _{\phi} \sup _{\alpha}\left\{\beta _ { k + 1 } \left[\frac{1}{2} a_{k+1} \| \psi_{k+1}\right.\right. \\
& \left.\quad-Q_{k+1} \phi\left\|^{2}+\frac{1}{2}\right\| \partial \phi\left\|^{2}+\frac{1}{2}\left\langle\alpha,|\phi|^{2}-1\right\rangle-\frac{1}{2 \lambda_{k+1}}\right\| \alpha\left\|^{2}+\frac{1}{2} v_{k+1}\right\| \phi-h \|^{2}\right] \\
& +\left[\frac{1}{2} c_{k+1}\left\|\psi_{k+1}-Q_{k+1} \phi\right\|^{2}+\frac{1}{2} b_{k+1}\|\partial \phi\|^{2}+\frac{1}{2} b_{k+1}\left\langle\alpha,|\phi|^{2}-1\right\rangle-\frac{d_{k+1}}{2 \lambda_{k+1}}\|\alpha\|^{2}\right. \\
& \left.\left.+\frac{1}{2} v_{k+1} e_{k+1}\|\phi-h\|^{2}\right]+\left(\beta_{k+1} a_{k+1}+c_{k+1}\right)\left\langle\left(z_{k+1}-1\right) \psi_{k+1}, \psi_{k+1}-Q_{k+1} \phi\right\rangle\right\} .
\end{aligned}
$$


The expression in the first square bracket above determines the new, renormalized main action. We take the corresponding minimal configuration $\phi_{k+1}$, which is a solution of the variational problem determined by the new action, and we expand the above expression around it, i.e. we take $\phi=\phi_{k+1}+\delta \phi, \alpha=\alpha_{k+1}+\delta \alpha$, and we have

$$
\begin{aligned}
& \beta_{k+1, u} A_{k+1, u}\left(\theta, \phi_{k+1, u}\right) \\
& =\beta_{k+1} A_{k+1}\left(\psi_{k+1}, \phi_{k+1}\right)+\left[\left(\beta_{k+1} a_{k+1}+c_{k+1}\right) \zeta_{k+1}\left\langle\psi_{k+1}, \psi_{k+1}-Q_{k+1} \phi_{k+1}\right\rangle\right. \\
& +\frac{1}{2} c_{k+1}\left\|\psi_{k+1}-Q_{k+1} \phi_{k+1}\right\|^{2}+\frac{1}{2} b_{k+1}\left\|\partial \phi_{k+1}\right\|^{2} \\
& \left.+\frac{2 b_{k+1}-d_{k+1}}{2 \lambda_{k+1}}\left\|\alpha_{k+1}\right\|^{2}+\frac{1}{2} v_{k+1} e_{k+1}\left\|\phi_{k+1}-h\right\|^{2}\right] \\
& +\beta_{k+1} \inf _{\delta \phi} \sup _{\delta \alpha}\left\{\left[\frac{1}{2}\left(a_{k+1}+\beta_{k+1}^{-1} c_{k+1}\right)\left\|\zeta_{k+1} \psi_{k+1}-Q_{k+1} \delta \phi\right\|^{2}\right.\right. \\
& +\frac{1}{2}\left(1+\beta_{k+1}^{-1} b_{k+1}\right)\|\partial \delta \phi\|^{2}+\frac{1}{2}\left(1+\beta_{k+1}^{-1} b_{k+1}\right)\left\langle\delta \phi,\left(\alpha_{k+1}+\delta \alpha\right) \delta \phi\right\rangle \\
& +\left(1+\beta_{k+1}^{-1} b_{k+1}\right)\left\langle\delta \phi, \delta \alpha \phi_{k+1}\right\rangle-\frac{1+\beta_{k+1}^{-1} d_{k+1}}{2 \lambda_{k+1}}\|\delta \alpha\|^{2} \\
& \left.+\frac{1}{2} v_{k+1}\left(1+\beta_{k+1}^{-1} e_{k+1}\right)\|\delta \phi\|^{2}\right]+\left[-\beta_{k+1}^{-1} c_{k+1}\left\langle\delta \phi, Q_{k+1}^{*}\left(\psi_{k+1}-Q_{k+1} \phi_{k+1}\right)\right\rangle\right. \\
& +\beta_{k+1}^{-1} b_{k+1}\left\langle\delta \phi,(-\Delta) \phi_{k+1}\right\rangle+\beta_{k+1}^{-1} b_{k+1}\left\langle\delta \phi, \alpha_{k+1} \phi_{k+1}\right\rangle \\
& \left.\left.+\frac{\beta_{k+1}^{-1} b_{k+1}-\beta_{k+1}^{-1} d_{k+1}}{\lambda_{k+1}}\left\langle\delta \alpha, \alpha_{k+1}\right\rangle+v_{k+1} \beta_{k+1}^{-1} e_{k+1}\left\langle\delta \phi, \phi_{k+1}-h\right\rangle\right]\right\} \text {. }
\end{aligned}
$$

Consider the variational problem above. Variational equations are

$$
\begin{aligned}
{[-(1+} & \left.\beta_{k+1}^{-1} b_{k+1}\right) \Delta+\left(a_{k+1}+\beta_{k+1}^{-1} c_{k+1}\right) Q_{k+1}^{*} Q_{k+1} \\
+ & \left.\left(1+\beta_{k+1}^{-1} b_{k+1}\right)\left(\alpha_{k+1}+\delta \alpha\right)+v_{k+1}\left(1+\beta_{k+1}^{-1} e_{k+1}\right)\right] \delta \phi+\left(1+\beta_{k+1}^{-1} b_{k+1}\right) \delta \alpha \phi_{k+1} \\
= & \left(a_{k+1}+\beta_{k+1}^{-1} c_{k+1}\right) Q_{k+1}^{*} \zeta_{k+1} \psi_{k+1}+\beta_{k+1}^{-1} c_{k+1} Q_{k+1}^{*}\left(\psi_{k+1}-Q_{k+1} \phi_{k+1}\right) \\
& +\beta_{k+1}^{-1} b_{k+1} \Delta \phi_{k+1}-\beta_{k+1}^{-1} b_{k+1} \alpha_{k+1} \phi_{k+1}-v_{k+1} \beta_{k+1}^{-1} e_{k+1}\left(\phi_{k+1}-h\right) \\
= & \left(a_{k+1}+\beta_{k+1}^{-1} c_{k+1}\right) Q_{k+1}^{*} \psi_{0}+\left(1+\beta_{k+1}^{-1} b_{k+1}\right) f_{0},
\end{aligned}
$$




$$
\begin{gathered}
\left(1+\beta_{k+1}^{-1} b_{k+1}\right)\left(\delta \phi \cdot \phi_{k+1}+\frac{1}{2}|\delta \phi|^{2}\right)-\frac{1+\beta_{k+1}^{-1} d_{k+1}}{\lambda_{k+1}} \delta \alpha \\
=-\frac{\beta_{k+1}^{-1} b_{k+1}-\beta_{k+1}^{-1} d_{k+1}}{\lambda_{k+1}} \alpha_{k+1}=\frac{1+\beta_{k+1}^{-1} d_{k+1}}{\lambda_{k+1}} \alpha_{0},
\end{gathered}
$$

where the last equalities are definitions of the functions $\psi_{0}, f_{0}, \alpha_{0}$. These equations can be written in the form

$$
\begin{gathered}
\left(-\Delta+a Q_{k+1}^{*} Q_{k+1}+v+\alpha_{k+1}+\delta \alpha\right) \delta \phi+\delta \alpha \phi_{k+1}=a Q_{k+1}^{*} \psi_{0}+f_{0}, \\
\delta \phi \cdot \phi_{k+1}+\frac{1}{2}|\delta \phi|^{2}-\frac{1}{\lambda} \delta \alpha=\frac{1}{\lambda} \alpha_{0},
\end{gathered}
$$

where

$$
\begin{gathered}
a=\frac{a_{k+1}+\beta_{k+1}^{-1} c_{k+1}}{1+\beta_{k+1}^{-1} b_{k+1}}=a_{k+1, u}, \quad v=v_{k+1} \frac{1+\beta_{k+1}^{-1} e_{k+1}}{1+\beta_{k+1}^{-1} b_{k+1}}=v_{k+1, u}, \\
\lambda=\lambda_{k+1} \frac{1+\beta_{k+1}^{-1} b_{k+1}}{1+\beta_{k+1}^{-1} d_{k+1}}=\lambda_{k+1, u} .
\end{gathered}
$$

The above system of equations is a special case of a basic system of equations (3.20) [2] studied in paper [2]. It is a minor modification of the system (2.17), and it has basically the same properties described in Proposition 3.1 [2]. If $\psi_{0}, \phi_{k+1}, \alpha_{k+1}, f_{0}, \alpha_{0}$ satisfy the assumptions of this proposition, then the system (4.5) has exactly one solution in the space of all complex configurations satisfying the only restriction $|\delta \alpha|<c_{6}$. We denote the solution by $\delta \phi_{k+1}, \delta \alpha_{k+1}$ again, though these functions are different from the solutions of (2.17) discussed in Sect. 2. We do not use those solutions in the rest of the paper, so this notation should not cause a confusion. The solution $\delta \phi_{k+1}, \delta \alpha_{k+1}$ is an analytic function of $\psi_{0}, \phi_{k+1}, \alpha_{k+1}, f_{0}, \alpha_{0}$ on the domain described above, and it satisfies the bounds

$$
\begin{aligned}
& \left|\delta \phi_{k+1}\left(\psi_{0}, f_{0}, \alpha_{0}\right)\right|,\left|\partial \delta \phi_{k+1}\left(\psi_{0}, f_{0}, \alpha_{0}\right)\right|,\left|\Delta \delta \phi_{k+1}\left(\psi_{0}, f_{0}, \alpha_{0}\right)\right|,\left|\delta \alpha_{k+1}\left(\psi_{0}, f_{0}, \alpha_{0}\right)\right| \\
& \quad \leqq K_{2} \max \left\{\left|\psi_{0}\right|,\left|f_{0}\right|,\left|\alpha_{0}\right|\right\} .
\end{aligned}
$$

The derivatives above, and in the previous formulas, are on the lattice $T_{L^{-1} \eta}$, but for simplicity we have omitted the superscript $L^{-1} \eta$ indicating the scale. Formulating these results we have assumed implicitly that the coefficients $a, v, \lambda$ are in a proper range, for example $\frac{1}{2}<a<\frac{3}{2}, 0<v \leqq 1, \lambda \geqq 1$. This is satisfied in our case, because they are equal to the unrenormalized coefficients $a_{k+1, u}, v_{k+1, u}, \lambda_{k+1, u}$ which satisfy these conditions. Let us check if the configurations $\psi_{0}, \phi_{k+1}, \alpha_{k+1}, f_{0}, \alpha_{0}$ satisfy the assumptions of Proposition 3.1 [2]. Obviously this depends on assumptions for the renormalization constants and the new variables $\psi_{k+1}, h$. Let us start with the first. Notice that the equations depend on these constants only through the combinations $\zeta_{k+1}, \beta_{k+1}^{-1} b_{k+1}, \beta_{k+1}^{-1} c_{k+1}, \beta_{k+1}^{-1} d_{k+1}, \beta_{k+1}^{-1} e_{k+1}$. For simplicity let us drop the subscript $k+1$ in the notations. We assume that these rescaled constants are 
sufficiently small, more precisely

$$
\left|\varepsilon_{k+1}^{-1} \zeta\right|,\left|\beta^{-1} b\right|,\left|\beta^{-1} c\right|,\left|\beta^{-1} d\right|,\left|\beta^{-1} e\right|<\varepsilon
$$

where $\varepsilon$ is a sufficiently small positive number. This implies that the coefficients $a_{k+1}, v_{k+1}, \lambda_{k+1}$ are correspondingly small variations of $a_{k+1, u}, v_{k+1, u}, \lambda_{k+1, u}$. We will come back to the discussion of these coefficients later on. Consider now the new variables. We need to consider at first real analytic functions of these variables, so we assume that

$$
\left(\psi_{k+1}, h\right) \in \Xi_{k+1}\left(1, \varepsilon_{k+1}\right) .
$$

Notice that the space above is defined by the renormalized functions $\phi_{k+1}, \alpha_{k+1}$. With this assumption, and by the definitions of $\psi_{0}, f_{0}, \alpha_{0}$ in (4.4) we have

$$
\begin{gathered}
\left|\psi_{0}\right|<\frac{3}{2} \varepsilon \varepsilon_{k+1}, \\
\left|f_{0}\right|<\frac{3}{2} \varepsilon \cdot \varepsilon_{k+1}+\frac{3}{2} \varepsilon \cdot \varepsilon_{k+1}+\frac{3}{2} \varepsilon \cdot \varepsilon_{k+1} \cdot \frac{3}{2}+\frac{3}{2} \varepsilon \sqrt{v_{k+1}} \varepsilon_{k+1}<7 \varepsilon_{k+1} \varepsilon, \\
\left|\alpha_{0}\right|<3 \varepsilon \cdot \varepsilon_{k+1}=3 \varepsilon_{k+1} \varepsilon,
\end{gathered}
$$

where we have estimated $\phi_{k+1}, \psi_{k+1}$ and various constants like $\left(1+\beta^{-1} b\right)^{-1},(1+$ $\left.\beta^{-1} d\right)^{-1},\left(a+\beta^{-1} c\right)\left(1+\beta^{-1} b\right)^{-1}=a_{k+1, u}$ simply by $\frac{3}{2}$. Assuming that $7 K_{2} \varepsilon_{k+1} \varepsilon \leqq$ $c_{6}$, the configurations $\psi_{0}, \phi_{k+1}, \alpha_{k+1}, f_{0}, \alpha_{0}$ satisfy the assumptions of Proposition 3.1 [2] and there exists exactly one solution of the system (4.4), or (4.5), with the properties described above. In particular it satisfies the bounds

$$
\left|\delta \phi_{k+1}\right|,\left|\partial \delta \phi_{k+1}\right|,\left|\Delta \delta \phi_{k+1}\right|,\left|\delta \alpha_{k+1}\right|<7 K_{2} \varepsilon_{k+1} \varepsilon
$$

on the domains (4.8), (4.9). It follows from the bounds, or even more clearly from Eqs. (4.4), that $\delta \phi_{k+1}, \delta \alpha_{k+1}$ are analytic functions of at least first order of the renormalization constants. Notice that up to now we do not have any strong restrictions on $\varepsilon$, the condition $K_{2} \varepsilon_{k+1} \varepsilon \leqq c_{6}$ is satisfied even with $\varepsilon=1$, we have stronger restrictions on $\varepsilon_{j}$ 's. The only other conditions we have used are that various constants like $\left(1+\beta^{-1} b\right)^{-1}$, etc. are close to 1 , or $<\frac{3}{2}$, and it is enough to take $\varepsilon \leqq \frac{1}{16}$. Thus we have constructed the expansion

$$
\phi_{k+1, u}=\phi_{k+1}+\delta \phi_{k+1}, \quad \alpha_{k+1, u}=\alpha_{k+1}+\delta \alpha_{k+1}
$$

of the unrenormalized minimal configurations around the renormalized ones, and the perturbations $\delta \phi_{k+1}, \delta \alpha_{k+1}$ are analytic functions of at least first order in the renormalization constants.

Let us come back to the expansion (4.3) and substitute there the solution of the system (4.4). We get

$$
\beta_{u} A_{k+1, u}\left(\theta, \phi_{k+1, u}\right)=\beta A_{k+1}\left(\psi_{k+1}, \phi_{k+1}\right)+\beta C^{(k+1)}+\frac{1}{2} v e\left(|h|^{2}-1\right)\left|T_{1}^{(k+1)}\right|,
$$


where, using Eqs. (4.4), the counterterm $C^{(k+1)}$ is given by

$$
\begin{aligned}
C^{(k+1)}= & C_{1}^{(k+1)}-C_{2}^{(k+1)} \\
= & \left(a+\beta^{-1} c\right) \zeta\left\langle\psi_{k+1}, \psi_{k+1}-Q_{k+1} \phi_{k+1}\right\rangle+\frac{1}{2} \beta^{-1} c\left\|\psi_{k+1}-Q_{k+1} \phi_{k+1}\right\|^{2} \\
& +\frac{1}{2} \beta^{-1} b\left\|\partial \phi_{k+1}\right\|^{2}+\frac{1}{2 \lambda}\left(2 \beta^{-1} b-\beta^{-1} d\right)\left\|\alpha_{k+1}\right\|^{2} \\
& +\frac{1}{2} v \beta^{-1} e\left\|\left|\phi_{k+1}\right|-1\right\|^{2}+v \beta^{-1} e\left\langle\left|\phi_{k+1}\right|, 1-\left(\phi_{k+1}\right)_{0} \cdot h\right\rangle \\
& -\left[-\frac{1}{2}\left(a+\beta^{-1} c\right) \zeta^{2}\left\|\psi_{k+1}\right\|^{2}+\frac{1}{2}\left(a+\beta^{-1} c\right)\left\|Q_{k+1} \delta \phi_{k+1}\right\|^{2}\right. \\
& +\frac{1}{2}\left(1+\beta^{-1} b\right)\left\|\partial \delta \phi_{k+1}\right\|^{2}+\frac{1}{2}\left(1+\beta^{-1} b\right)\left\langle\delta \phi_{k+1},\left(\alpha_{k+1}+2 \delta \alpha_{k+1}\right) \delta \phi_{k+1}\right\rangle \\
& -\frac{1}{2 \lambda}\left(1+\beta^{-1} d\right)\left\|\delta \alpha_{k+1}\right\|^{2}+\left(1+\beta^{-1} b\right)\left\langle\delta \phi_{k+1}, \delta \alpha_{k+1} \phi_{k+1}\right\rangle \\
& \left.+\frac{1}{2} v\left(1+\beta^{-1} e\right)\left\|\delta \phi_{k+1}\right\|^{2}\right],
\end{aligned}
$$

where $a, v, \lambda$ denote $a_{k+1}, v_{k+1}, \lambda_{k+1}$ now. The above expression is a local function of the configurations $\psi_{k+1}, \phi_{k+1}, \delta \phi_{k+1}, \delta \alpha_{k+1}$, so we can write a decomposition as in (2.28) restricting the norms and scalar products to blocks $B_{k+1}(z), z \in T_{1}^{(k+1)}$. We get

$$
C^{(k+1)}=\sum_{z \in T_{1}^{(k+1)}} C^{(k+1)}(z)
$$

and the functions $C^{(k+1)}(z)$ satisfy the usual Euclidean covariance and $O(N)$ invariance properties. Of course the same holds for $C_{1}^{(k+1)}$ and $C_{2}^{(k+1)}$ separately. These functions are analytic on the domains (4.8), (4.9), and $C_{1}^{(k+1)}$ is at least of first order in the renormalization constants, $C_{2}^{(k+1)}$ is at least of second order. have

The expansion (4.12) determines corresponding expansions of $\psi_{k+1, u}^{(j)}$, i.e. we

$$
\psi_{k+1, u}^{(j)}=\psi_{k+1}^{(j)}+\delta \psi_{k+1}^{(j)}
$$

where

$\delta \psi_{k+1}^{(j)}=Q_{j} \delta \phi_{k+1}+\frac{1-L^{-2 j}}{1-L^{-2(k+1)}}\left(L^{j-1} \eta\right)^{2} Q_{k+1-j}^{*}\left(\zeta_{k+1} \psi_{k+1}-Q_{k+1} \delta \phi_{k+1}\right)$.

They cause further changes in the effective action and the generating functional, we expand them around the new configurations and include perturbations into the new contribution to the effective action and the generating functional correspondingly. 
We define an unrenormalized $k+1^{\text {st }}$ effective action $\mathscr{E}_{u}^{(k+1)}$ by the formula

$$
\begin{aligned}
\mathscr{E}_{u}^{(k+1)}\left(\psi_{k+1}, h\right)= & \mathscr{E}_{0}^{(k+1)}\left(z_{k+1} \psi_{k+1}, h\right)+\sum_{j=1}^{k}\left[\mathscr { E } ^ { ( j ) } \left(\psi_{k+1}^{(j)}\left(\psi_{k+1}, h\right)\right.\right. \\
& \left.\left.+\delta \psi_{k+1}^{(j)}\left(\psi_{k+1}, h\right), h\right)-\mathscr{E}^{(j)}\left(\psi_{k+1}^{(j)}\left(\psi_{k+1}, h\right), h\right)\right]
\end{aligned}
$$

The $k+1^{\text {st }}$ contribution to the generating functional is defined finally by the formula

$$
\begin{aligned}
& \mathscr{F}^{(k+1)}\left(\psi_{k+1}, h, g\right)=\left\langle g, \mathscr{M}^{(k+1)}\left(\psi_{k+1}, h, g\right)\right\rangle_{1} \\
& \mathscr{M}^{(k+1)}\left(\psi_{k+1}, h, g\right)= \beta_{k}^{-\frac{1}{2}} \mathscr{M}_{0}^{(k+1)}\left(z_{k+1} \psi_{k+1}, h, g\right)+\delta \phi_{k+1}+\sum_{j=1}^{k}\left[\mathscr { M } ^ { ( j ) } \left(\psi_{k+1}^{(j)}\left(\psi_{k+1}, h\right)\right.\right. \\
&\left.\left.+\delta \psi_{k+1}^{(j)}\left(\psi_{k+1}, h\right), h, g\right)-\mathscr{M}^{(j)}\left(\psi_{k+1}^{(j)}\left(\psi_{k+1}, h\right), h, g\right)\right] .
\end{aligned}
$$

With the above definitions of the new contributions the effective action $\mathscr{E}_{k}\left(\psi^{(k)}\right.$ $\left.\left(\psi_{k+1}\right), h\right)$ has the representation (H.2) with the terms $\mathscr{E}^{(j)}\left(y ; \psi_{k+1}^{(j)}\left(\psi_{k+1}, h\right), h\right)$ determined by the functions $\mathscr{E}^{(j)}\left(y ; \psi_{j}, h\right)$. The fact that these functions satisfy all the inductive assumptions follows immediately from the inclusions

$$
\Xi_{j}^{c}\left(\mathbb{B}_{j}(X) ; 1, \varepsilon_{j}\right) \subset \Xi_{j, u}^{c}\left(\mathbb{B}_{j}(X) ; 1, \varepsilon_{j, u}\right),
$$

where $\varepsilon_{j}$ is defined by the formula in (H.1) with the sum over $n$ starting with $n=k+1$, and $\varepsilon_{j, u}$ is defined exactly by that formula. Thus $\varepsilon_{j, u}=\varepsilon_{j}+\frac{1}{k^{2}} \alpha_{0} \xi^{\alpha}>$ $\varepsilon_{j}+\frac{1}{k^{2}} \frac{1}{3} \varepsilon_{j}$. We postpone a proof of the above inclusions to the paper on localization expansions. This remark applies also to the terms of the generating functional $\mathscr{F}_{k}\left(\psi^{(k)}\left(\psi_{k+1}\right), h, g\right)$.

The first term on the right-hand side of (4.17) has the localization expansion described in Proposition 2.1, and we have to construct such a representation for the sum in (4.17). We cannot use the local representations of the actions $\mathscr{E}^{(j)}$ for the same reason as in the case of the fluctuation integral contribution (2.55), namely we need the whole resummed $\mathscr{E}^{(j)}$ in order to apply the representation (3.127) [1] in terms of irrelevant expressions. To construct the representation (2.56) we have used the first order expansion in the fluctuation variable in (2.57), which yields also a first order expansion in the constant $\beta_{k}^{-\frac{1}{2}}$. Here we may use Eqs. (4.5) and formula (4.16) to construct a first order expansion in the renormalization constants. Actually we need an expansion in some functions defined on lattices, so that we can localize it as in (2.57). Let us recall that the coefficients of Eqs. (4.5) are equal to the unrenormalized coefficients $a_{k+1, u}, v_{k+1, u}, \lambda_{k+1, u}$ so the only dependence on the renormalization constants is through the functions $\psi_{0}, f_{0}, \alpha_{0}$, and they are at least of first order in the constants. The solution $\delta \phi_{k+1}\left(\psi_{0}, f_{0}, \alpha_{0}\right), \delta \alpha_{k+1}\left(\psi_{0}, f_{0}, \alpha_{0}\right)$, which is an analytic function of $\psi_{0}, f_{0}, \alpha_{0}$, is of first order in these variables, i.e., $\delta \phi_{k+1}(0,0,0)=0, \delta \alpha_{k+1}(0,0,0)=0$, so we can expand in functions $\psi_{0}, f_{0}, \alpha_{0}$. The function $\delta \psi_{k+1}^{(j)}$ given by the formula (4.16) depends on $\psi_{0}, f_{0}, \alpha_{0}$, and it is equal to 0 if all these configurations are equal to 0 . Let us write this dependence explicitly, i.e., we write $\delta \psi_{k+1}^{(j)}\left(\psi_{0}, f_{0}, \alpha_{0}\right)$, and we have $\delta \psi_{k+1}^{(j)}(0,0,0)=0$. Using these properties we can write the differences in (4.17) as integrals of a derivative with respect to a parameter $t$ multiplying the configurations $\psi_{0}, f_{0}, \alpha_{0}$, and this derivative can be 
expressed in terms of derivatives with respect to these configurations. The obtained expressions can be localized then in cubes $\Delta(z)=B_{k+1}(z)$. This way we can write $\mathscr{E}_{u}^{(k+1)}$ as a sum of the local expressions over points of the lattice $T_{1}^{(k+1)}$ as in (2.56), and the local functions are given by the formula

$$
\begin{aligned}
\mathscr{E}_{u}^{(k+1)}(z)= & \mathscr{E}_{0}^{(k+1)}\left(z ; z_{k+1} \psi_{k+1}, h\right)+\sum_{j=1}^{k} \int_{0}^{1} d t \frac{d}{d \tau} \mathscr{E}^{(j)}\left(\psi_{k+1}^{(j)}+\delta \psi_{k+1}^{(j)}\left(\left(t+\tau \chi_{\Delta(z)}\right) \psi_{0}\right.\right. \\
& \left.\left.\left(t+\tau \chi_{\Delta(z)}\right) f_{0},\left(t+\tau \chi_{\Delta(z)}\right) \alpha_{0}\right), h\right)\left.\right|_{\tau=0}
\end{aligned}
$$

The above functions are Euclidean covariant with respect to transformations of the lattice $T_{1}^{(k+1)}$, and they are invariant with respect to the orthogonal group. This ends the first part of the renormalization operation.

A result of this operation can be written in the following way:

$$
\begin{aligned}
& \chi_{k+1, u}\left(S^{(k)} T^{(k)} \chi^{(k)} \rho_{k}\right)\left(\psi_{k+1}\right) \\
& =\chi_{k+1, u} \exp \left[-\beta_{k+1} A_{k+1}\left(\psi_{k+1}, \phi_{k+1}\right)+\sum_{j=1}^{k} \sum_{y \in T_{L^{(j-1}}} \mathscr{E}^{(j)}\left(y ; \psi_{k+1}^{(j)}\left(\psi_{k+1}, h\right), h\right)\right. \\
& \quad+\sum_{z \in T_{1}^{(k+1)}}\left\{\mathscr{E}_{u}^{(k+1)}\left(z ; \psi_{k+1}, h\right)-\beta_{k+1} C^{(k+1)}\left(z ; \psi_{k+1}, h\right)-E^{(k+1)}(z)\right\}-E_{k+1}\left|T_{1}^{(k+1)}\right| \\
& \left.\quad+\left\{\left\langle g, \phi_{k+1}\right\rangle+\sum_{j=1}^{k} \mathscr{F}^{(j)}\left(\psi_{k+1}^{(j)}\left(\psi_{k+1}, h\right), h, g\right)+\mathscr{F}^{(k+1)}\left(\psi_{k+1}, h, g\right)\right\}\right],
\end{aligned}
$$

where

$$
E^{(k+1)}(z)=\mathscr{E}_{u}^{(k+1)}\left(z ; e_{1}, e_{1}\right)-\beta_{k+1} C^{(k+1)}\left(z ; e_{1}, e_{1}\right),
$$

and $E^{(k+1)}(z)$ does not depend on $z$ by the translation invariance,

$$
E_{k+1}=E_{k}^{\prime \prime \prime} L^{d}-E^{(k+1)}(0) .
$$

The density defined by the exponential on the right-hand side of (4.21) has the form required by the inductive assumptions for $k+1$. We have to verify that it has all the properties required by the assumptions. The "old" contributions satisfy all the assumptions, except that we have to prove the inclusion (4.19). With the new contributions we face the same problems as with the contributions coming from the fluctuation integral, we have to verify that they are analytic on proper domains, and we have to construct localization expansions satisfying proper bounds. The formula (4.21) determines the new contribution to the effective action. It is given by the sum over $z \in T_{1}^{(k+1)}$ of the local functions

$$
\begin{aligned}
\mathscr{E}^{(k+1)}\left(z ; \psi_{k+1}, h\right)= & \mathscr{E}_{u}^{(k+1)}\left(z ; \psi_{k+1}, h\right)-\beta_{k+1} C^{(k+1)}\left(z ; \psi_{k+1}, h\right)-E^{(k+1)}(z), \\
& z \in T_{1}^{(k+1)} .
\end{aligned}
$$

They are functions of $\left(\psi_{k+1}, h\right)$ and the renormalization constants $\zeta, \beta^{-1} b, \beta^{-1} c$, $\beta^{-1} d, \beta^{-1} e$, and we do not know yet their domains of definition, we do not know 
even if they are defined on the space (4.9). They are certainly defined on sufficiently small domains, and satisfy the symmetry properties in (H.3). We have to prove (H.4), which implies the analyticity statement in (H.2), and we have to prove the renormalization conditions (H.5). This is achieved by a proper choice of the renormalization constants. The conditions in (H.5) determine a system of equations for the constants, and we will prove that this system has a unique small solution. Substituting this solution into the functions (4.22) yields the final contribution to the effective action, satisfying all the inductive hypotheses (H.2)-(H.5). We will prove also that the above solution satisfies the inductive hypothesis (H.6). Finally, we have to prove that the new contribution to the generating functional satisfies the hypothesis (H.7). In this section we formulate propositions on localization expansions for the new contributions. These propositions will be proved in the same paper as Propositions 2.1, 2.2. In the next section we will analyze the renormalization group equations for the renormalization constants, and we will prove all the above mentioned properties. This will end the renormalization operation and the proof of the basic theorems.

Let us start with a proposition on the functions in (4.22).

Proposition 4.1. If the constant $\varepsilon$ in (4.8) is small enough, then the functions $C_{1}^{(k+1)}(z), C_{2}^{(k+1)}(z), \mathscr{E}_{u}^{(k+1)}(z)$ defined by the formulas (4.14), (4.15), (4.20) have localization expansions satisfying all the properties in the inductive hypothesis (H.4) for $j=k+1$, but with the constant $E_{0}$ in the bounds replaced by $C_{4} \varepsilon_{k+1}^{2} \varepsilon$, $C_{4} \varepsilon_{k+1}^{2} \varepsilon^{2}, N L^{d} B_{4}+1+C_{5} E_{0} \varepsilon$ correspondingly, where $C_{4}, C_{5}$ are positive "absolute" constants. Terms of the expansions can be extended to analytic functions of the variables $\left(\psi_{k+1}, h\right)$ and the renormalization constants, defined on the spaces

$$
\Xi_{k+1}^{c}\left(\mathbb{B}_{k+1}(X) ; 1, \varepsilon_{k+1}\right) \times\{\text { the polydisc }(4.8)\},
$$

and depending on the variables restricted to the domains $X$.

This proposition yields also localization expansions of the whole new contributions $\mathscr{E}^{(k+1)}(z)$ defined by (4.22), but with bounds depending on $\beta_{k+1}$ and $\varepsilon$. We will come back to a discussion of the expansion in the next section, when we will determine the renormalization constants, because only then we will obtain a precise bound $\varepsilon$ on the constants. For the functions $\mathscr{E}_{u}^{(k+1)}(z)$ we can assume now that

$$
C_{5} E_{0} \varepsilon \leqq 1
$$

and we obtain the bounds in (H.4) with the constant $N L^{d} B_{4}+2=B_{6}$. We will discuss the above assumption in the next section also. The next proposition concerns the new contribution to the generating functional.

Proposition 4.2. For $\varepsilon$ small enough the functions $\mathscr{M}^{(k+1)}(x ; g)$ defined by the formula (4.18) have localization expansions satisfying all the properties in the hypothesis (H.7) for $j=k+1$, but with the constant $c_{9}$ in the bounds replaced by $\frac{1}{2} \beta^{-\frac{1}{4}}+C_{6} \varepsilon L^{(k+1)\left(2 \gamma-\alpha_{1}\right)}$, where $C_{6}$ is another "absolute" constant Terms of the expansions can be analytically extended onto products of the spaces (4.23) and $\left\{g:\|g\|_{\ell^{1}}<1\right\}$, and they depend on the variables restricted to the corresponding domains. 
It follows from this proposition that to complete the proof of (H.7) for the new contribution $\mathscr{F}^{(k+1)}(g)$ we have to prove that the expression $C_{6} \varepsilon L^{(k+1)\left(2 \gamma-\alpha_{1}\right)}$ is arbitrarily small for $\beta$ large enough. This will follow again from the bounds on the renormalization constants in the next section. The above propositions will be proved together with all other results on localization expansions.

\section{The Renormalization Conditions, and the Conclusion of the $k+1^{\text {st }}$ Step}

We start with an analysis of the renormalization conditions (H.5). Consider the first three conditions there. They are formulated in terms of the effective potential of the action $\mathscr{E}^{(k+1)}$, i.e. we take a constant configuration $\psi_{k+1}=\psi$ and define the effective potential $\mathscr{V}^{(k+1)}(\psi, h)$ by the equality $(2.25)$ [1]. We distinguish three contributions to this function, one from the action $\mathscr{E}_{u}^{(k+1)}$, one from the expression in the first square bracket in (4.14), which is denoted by $C_{1}^{(k+1)}$, and one from the expression in the second square bracket in (4.14) denoted by $C_{2}^{(k+1)}$. We have

$$
\mathscr{E}_{u}^{(k+1)}(z ; \psi, h)=\mathscr{V}_{u}^{(k+1)}(\psi, h)=f_{u}^{(k+1)}\left(U, V, W^{2}\right),
$$

and we are interested in the function $f_{u}^{(k+1)}(u, v, 0)$. It is an analytic function of $u, v$ on the discs $\left\{|u-1|<\frac{1}{3} \delta_{0}\right\},\left\{|v-1|<\frac{\delta_{0}^{2}}{v_{k+1}}\right\}$, where we can take $\delta_{0}=\frac{1}{2} K_{1}^{-1} \varepsilon_{k+1}$ by Lemma 3.2 [1]. It is also an analytic function of the renormalization constants on the polydisc (4.8). For simplicity of formulations let us introduce the notations

$$
\begin{array}{ll}
\xi_{1}=\frac{1}{\varepsilon_{k+1}} \zeta_{k+1}, & \xi_{2}=\beta_{k+1}^{-1} e_{k+1}, \quad \xi_{3}=\beta_{k+1}^{-1} b_{k+1}, \\
\xi_{4}=\beta_{k+1}^{-1} c_{k+1}, & \xi_{5}=\beta_{k+1}^{-1} d_{k+1} .
\end{array}
$$

The function $f_{u}^{(k+1)}$ is analytic in $\xi$ on the polydisc $\left\{\left|\xi_{i}\right|<\varepsilon\right\}$ with $\varepsilon$ sufficiently small, e.g. $\varepsilon \leqq c_{10}, \quad c_{10}$ given by taking the equality in (4.24). On the whole analyticity domain it satisfies the bound $\left|f_{u}^{(k+1)}\right|<B_{6} K_{0}$, hence its derivatives satisfy the bounds

$$
\begin{aligned}
& \left|\left(\frac{\partial}{\partial u} f_{u}^{(k+1)}\right)(1,1,0)\right|<B_{6} K_{0} \frac{3}{\delta_{0}}=6 B_{6} K_{0} K_{1} \frac{1}{\varepsilon_{k+1}}, \\
& \left|\left(\frac{\partial}{\partial v} f_{u}^{(k+1)}\right)(1,1,0)\right|<B_{6} K_{0} \frac{v_{k+1}}{\delta_{0}^{2}}=4 B_{6} K_{0} K_{1}^{2} \frac{v_{k+1}}{\varepsilon_{k+1}^{2}} .
\end{aligned}
$$

The functions $C_{p}^{(k+1)}(z ; \psi, h), \quad p=1,2$ have similar representations in terms of functions $c_{p}^{(k+1)}\left(u, v, w^{2}\right)$ and we have to calculate the corresponding derivatives. For the function $c_{1}^{(k+1)}$ we calculate them explicitly using formulas for solutions of the variational problems for constant configurations. In particular we have 
$\phi_{k+1}\left(u e_{1}, v e_{1}\right)=A_{k+1}(u, v) e_{1}$, and

$$
\begin{gathered}
A_{k+1}(1,1)=1,\left(\frac{\partial}{\partial u} A_{k+1}\right)(1,1)=\frac{a_{k+1}}{\lambda_{k+1}} \frac{1}{1+\frac{a_{k+1}+v_{k+1}}{\lambda_{k+1}}}, \\
\left(\frac{\partial}{\partial v} A_{k+1}\right)\left(1, v_{k+1}\right)=\frac{1}{\lambda_{k+1}} \cdot \frac{1}{1+\frac{a_{k+1}+v_{k+1}}{\lambda_{k+1}}} v_{k+1} .
\end{gathered}
$$

From these formulas and the definition of $c_{1}^{(k+1)}$ we obtain easily

$$
\begin{gathered}
\left(\frac{\partial}{\partial u} c_{1}^{(k+1)}\right)(1,1,0)=\left(a_{k+1}+\beta_{k+1}^{-1} c_{k+1}\right) \zeta_{k+1}^{2}+\left(a_{k+1}+\beta_{k+1}^{-1} c_{k+1}\right) \zeta_{k+1} \frac{1+\frac{v_{k+1}}{\lambda_{k+1}}}{1+\frac{a_{k+1}+v_{k+1}}{\lambda_{k+1}}} \\
\left(\frac{\partial}{\partial v} c_{1}^{(k+1)}\right)(1,1,0)=-\left(a_{k+1}+\beta_{k+1}^{-1} c_{k+1}\right) \zeta_{k+1} \frac{1}{\lambda_{k+1}} \cdot \frac{1}{1+\frac{a_{k+1}+v_{k+1}}{\lambda_{k+1}}} v_{k+1} \\
-\beta_{k+1}^{-1} e_{k+1} v_{k+1} .
\end{gathered}
$$

We do not need to calculate the derivatives of $c_{2}^{(k+1)}$ explicitly, because they are at least of second order in the renormalization constants, we need only bounds for them, which will be discussed later. We can write now the three renormalization conditions in (H.5). Notice that the first condition is satisfied by the choice of the constant $E^{(k+1)}$. Let us simplify the notation omitting again the subscript " $k+1$ " for all constants. The remaining two renormalization conditions can be written in the form

$$
\begin{aligned}
& \left(\frac{\partial}{\partial u} f_{u}^{(k+1)}\right)(1,1,0)-\beta\left[\left(a+\beta^{-1} c\right) \zeta^{2}+\left(a+\beta^{-1} c\right) \frac{1+\frac{v}{\lambda}}{1+\frac{a+v}{\lambda}} \zeta\right] \\
& \quad+\beta\left(\frac{\partial}{\partial u} c_{2}^{(k+1)}\right)(1,1,0)=0, \\
& \frac{1}{v}\left(\frac{\partial}{\partial v} f_{u}^{(k+1)}\right)(1,1,0)+\beta\left[\left(a+\beta^{-1} c\right) \frac{1}{\lambda} \frac{1}{1+\frac{a+v}{\lambda}} \zeta+\beta^{-1} e\right] \\
& \quad+\beta \frac{1}{v}\left(\frac{\partial}{\partial v} c_{2}^{(k+1)}\right)(1,1,0)=0 .
\end{aligned}
$$

We obtain two equations for the five unknown renormalization constants.

Now we consider the last condition in (H.5). It is much more difficult to analyze because we have to calculate second order derivatives with respect to the variables $\psi_{k+1}$, and we have to do it explicitly for the function $C_{1}^{(k+1)}$. We are interested in the derivatives calculated at the constant configuration $\psi_{k+1}=e_{1}, h=e_{1}$, so we write $\psi_{k+1}=e_{1}+\psi^{\prime}$ and we calculate the derivatives with respect to $\psi^{\prime}$ at $\psi^{\prime}=0$. The function $C_{1}^{(k+1)}$ is an analytic function of $\psi^{\prime}$ in a neighborhood of 0 , and it is enough to expand it in $\psi^{\prime}$ up to second order terms. From the form of the function $C_{1}^{(k+1)}$ it is clear that we have to find an expansion of $\phi_{k+1}\left(e_{1}+\psi^{\prime}, e_{1}\right)$ up to second 
order in $\psi^{\prime}$, and an expansion of $\alpha_{k+1}\left(e_{1}+\psi^{\prime}, e_{1}\right)$ up to first order in $\psi^{\prime}$. We write

$$
\begin{aligned}
\phi_{k+1}\left(e_{1}+\psi^{\prime}, v e_{1}\right) & =e_{1}+\phi_{k+1}^{(1)}\left(\psi^{\prime}\right)+\phi_{k+1}^{(2)}\left(\psi^{\prime}\right)+\cdots, \alpha_{k+1}\left(e_{1}+\psi^{\prime}, v e_{1}\right) \\
& =\alpha_{k+1}^{(1)}\left(\psi^{\prime}\right)+\cdots,
\end{aligned}
$$

and by (2.21), (2.22) we have

$$
\begin{gathered}
\phi_{k+1}^{(1)}\left(\psi^{\prime}\right)=a_{k+1} G_{k+1} Q_{k+1}^{*} \psi^{\prime}-G_{k+1}\left(\frac{1}{\lambda_{k+1}}+G_{k+1}\right)^{-1} a_{k+1} G_{k+1} Q_{k+1}^{*}\left(e_{1} \otimes e_{1}\right) \psi^{\prime} \\
\alpha_{k+1}^{(1)}\left(\psi^{\prime}\right)=\left(\frac{1}{\lambda_{k+1}}+G_{k+1}\right)^{-1} a_{k+1} G_{k+1} Q_{k+1}^{*} e_{1} \cdot \psi^{\prime}
\end{gathered}
$$

Differentiating Eqs. (2.17) up to the second order in $\psi^{\prime}$ and solving the resulting equations we obtain a formula for $\phi_{k+1}^{(2)}\left(\psi^{\prime}\right)$. To write it in a simple form we introduce the notations

$$
\begin{aligned}
H_{k+1, t r} \psi^{\prime} & =a_{k+1} G_{k+1} Q_{k+1}^{*}\left(I-e_{1} \otimes e_{1}\right) \psi^{\prime}, \\
H_{k+1, \text { long }} \psi^{\prime} & =a_{k+1} G_{k+1} Q_{k+1}^{*}\left(e_{1} \otimes e_{1}\right) \psi^{\prime},
\end{aligned}
$$

and we have then

$$
\begin{aligned}
\phi_{k+1}^{(2)}\left(\psi^{\prime}\right)= & -e_{1} G_{k+1}\left(G_{k+1}+\frac{1}{\lambda_{k+1}}\right)^{-1} \\
& \times\left[\left(H_{k+1, t r} \psi^{\prime}\right) \cdot\left(H_{k+1, t r} \psi^{\prime}\right)+\frac{3}{\lambda_{k+1}^{2}}\left(H_{k+1, \text { long }} \psi^{\prime}\right) \cdot\left(H_{k+1, \text { long }} \psi^{\prime}\right)\right] \\
& -2 G_{k+1}\left(\left(e_{1} \cdot H_{k+1, \text { long }} \psi^{\prime}\right) H_{k+1, t r} \psi^{\prime}\right) .
\end{aligned}
$$

Substituting expansions (5.6) into formula (4.14) for the function $C_{1}^{(k+1)}$ we obtain an expansion of this function, and second order terms of this expansion yield a quadratic form in $\psi^{\prime}$, which we write in the simplified form omitting the subscript " $k+1 "$,

$$
\begin{aligned}
& \frac{1}{2}\left(a+\beta^{-1} c\right) \zeta^{2}\left\|\psi^{\prime}\right\|^{2}-\frac{1}{2}\left(a+\beta^{-1} c\right) \zeta\left\langle e_{1}, \phi^{(2)}\left(\psi^{\prime}\right)\right\rangle+\left(a+\beta^{-1} c\right) \zeta\left\langle\psi^{\prime}, \psi^{\prime}-Q \phi^{(1)}\left(\psi^{\prime}\right)\right\rangle \\
& \quad+\frac{1}{2} \beta^{-1} c\left\|\psi^{\prime}-Q \phi^{(1)}\left(\psi^{\prime}\right)\right\|^{2}+\frac{1}{2} \beta^{-1} b\left\|\partial \phi^{(1)}\left(\psi^{\prime}\right)\right\|^{2} \\
& \quad+\frac{1}{2 \lambda}\left(2 \beta^{-1} b-\beta^{-1} d\right)\left\|\alpha^{(1)}\left(\psi^{\prime}\right)\right\|^{2}+\frac{1}{2} \beta^{-1} e\left\|\phi^{(1)}\left(\psi^{\prime}\right)\right\|^{2} .
\end{aligned}
$$

From the above formula and (5.7),(5.9) it is easy to write explicitly the operator of this quadratic form. We need a kernel of this operator to calculate the constant in (H.5) only. This constant is expressed in terms of transversal components of the kernel, so we need only contributions from transversal components of $\psi^{\prime}$ in (5.7), (5.9). Denoting $\psi_{t r}^{\prime}=\left(I-e_{1} \otimes e_{1}\right) \psi^{\prime}=\theta$ we obtain the following formula 
for the transversal part of the above quadratic form:

$$
\begin{aligned}
\frac{1}{2}(a+ & \left.\beta^{-1} c\right) \zeta^{2}\|\theta\|^{2}+\frac{1}{2}\left(a+\beta^{-1} c\right) \frac{1}{1+\frac{a+v}{\lambda}} \zeta\|H \theta\|^{2}+\left(a+\beta^{-1} c\right) \zeta\langle\theta, \theta-Q H \theta\rangle \\
& +\frac{1}{2} \beta^{-1} c\|\theta-Q H \theta\|^{2}+\frac{1}{2} \beta^{-1} b\|\partial H \theta\|^{2}+\frac{1}{2} v \beta^{-1} e\|H \theta\|^{2} \\
= & \frac{1}{2}\left(a+\beta^{-1} c\right) \zeta^{2}\|\theta\|^{2}+\left(a+\beta^{-1} c\right) \zeta\left\langle\theta,\left(I-a Q G Q^{*}\right) \theta\right\rangle \\
& +\frac{1}{2} \beta^{-1} c\left\langle\theta,\left(I-a Q G Q^{*}\right)^{2} \theta\right\rangle+\frac{1}{2} a^{2}\left(\frac{a+\beta^{-1} c}{1+\frac{a+v}{\lambda}} \zeta+v \beta^{-1} e-v \beta^{-1} b\right)\left\langle\theta, Q G^{2} Q^{*} \theta\right\rangle \\
& +\frac{1}{2} a^{2} \beta^{-1} b\left\langle\theta, Q G(-\Delta+v) G Q^{*} \theta\right\rangle .
\end{aligned}
$$

This gives also a formula for the kernel of the operator. Actually we need only components in the direction of the vector $e_{2}$, so we may take $\theta=\theta_{2} e_{2}$, which amounts to taking a scalar function $\theta$ in the above formula. The kernel is a translationally invariant function of the unit lattice points, we have to take its infinite volume limit, calculate a Fourier transform of the limit, and the derivative in (H.5). To do this calculation we use the representations derived in [3]. We have

$$
G=(-\Delta+v)^{-1}-a(-\Delta+v)^{-1} Q^{*}\left(I+a Q(-\Delta+v)^{-1} Q^{*}\right)^{-1} Q(-\Delta+v)^{-1},
$$

and from this we obtain the formulas

$$
\begin{gathered}
I-a Q G Q^{*}=\left(I+a Q(-\Delta+v)^{-1} Q^{*}\right)^{-1}, \\
Q G^{2} Q^{*}=\left(Q(-\Delta+v)^{-2} Q^{*}\right)\left(I+a Q(-\Delta+v)^{-1} Q^{*}\right)^{-2}, \\
Q G(-\Delta+v) G Q^{*}=\left(Q(-\Delta+v)^{-1} Q^{*}\right)\left(I+a Q(-\Delta+v)^{-1} Q^{*}\right)^{-2} .
\end{gathered}
$$

They are written in terms of three basic unit lattice operators. They are translationally invariant, so they are convolution operators and therefore they commute. For their Fourier transforms we have the following representations:

$$
\begin{aligned}
f(p) & =\left(I+a Q(-\Delta+v)^{-1} Q^{*}\right)^{-1}(p) \\
& =\left(1+a \sum_{l} \frac{\left|u_{m}(p+l)\right|^{2}}{\Delta(p+l)+v}\right)^{-1}=(1+a g(p))^{-1}, \\
g(p) & =\left(Q(-\Delta+v)^{-1} Q^{*}\right)(p)=\sum_{l} \frac{\left|u_{m}(p+l)\right|^{2}}{\Delta(p+l)+v}, \\
h(p) & =\left(Q(-\Delta+v)^{-2} Q^{*}\right)(p)=\sum_{l} \frac{\left|u_{m}(p+l)\right|^{2}}{(\Delta(p+l)+v)^{2}},
\end{aligned}
$$


where $p \in \mathbb{R}^{d},\left|p_{\mu}\right| \leqq \pi, l=2 \pi n, n \in \mathbb{Z}^{d},\left|n_{\mu}\right| \leqq \frac{1}{2}\left(L^{m}-1\right)$,

$$
\begin{gathered}
\Delta(q)=\sum_{\mu=1}^{d}\left|\partial_{\mu}(q)\right|^{2}, \quad \partial_{\mu}(q)=L^{m}\left(e^{i L^{-m} q_{\mu}}-1\right) \\
u_{m}(q)=\prod_{\mu=1}^{d} u_{m, \mu}(q), \quad u_{m, \mu}(q)=\frac{e^{i q_{\mu}}-1}{\partial_{\mu}(q)}, \quad m=k+1 \quad \text { in our case } .
\end{gathered}
$$

The Fourier transforms of the kernels of the operators (5.12) are equal to $f(p), h(p)$ $f^{2}(p), g(p) f^{2}(p)$ correspondingly. By (H.5) we have to calculate the second order derivatives with respect to $p_{1}$ at $p=0$ for these functions, and also for $f^{2}(p)$. Take $p=t e_{1}$ and write simply $f(t), g(t), h(t)$. From the above formulas we can calculate easily the values

$$
\begin{gathered}
f(0)=\frac{v}{a+v}, \quad f^{\prime}(0)=0, \\
f^{\prime \prime}(0)=2 \frac{a}{(a+v)^{2}}\left(1+\frac{1}{12}\left(1-L^{-2 m}\right) v-v^{2} c_{1}(v)\right), \\
g(0)=\frac{1}{v}, \quad g^{\prime}(0)=0, \quad g^{\prime \prime}(0)=-2 \frac{1}{v^{2}}\left(1+\frac{1}{12}\left(1-L^{-2 m}\right) v-v^{2} c_{1}(v)\right), \\
h(0)=\frac{1}{v^{2}}, \quad h^{\prime}(0)=0, \quad h^{\prime \prime}(0)=-2 \frac{1}{v^{3}}\left(2+\frac{1}{12}\left(1-L^{-2 m}\right) v-v^{3} c_{2}(v)\right),
\end{gathered}
$$

where

$$
c_{i}(v)=\sum_{l \neq 0} \frac{1}{2 L^{2 m}\left(1-\cos L^{-m} l\right)} \cdot \frac{1}{\left[2 L^{2 m}\left(1-\cos L^{-m} l\right)+v\right]^{i}}, \quad i=1,2,
$$

the summation is over a one-component index $l$. Notice the following simple properties of these numbers: $0<c_{i}(v)<\frac{1}{20}, 0<c_{1}(v)-v c_{2}(v)<c_{1}(v)<\frac{1}{20}$. With the help of these formulas we can calculate explicitly the constant in (H.5) for the kernel of the quadratic form (5.11). Taking the constants for kernels of the second order derivatives of the functions $\mathscr{E}_{u}^{(k+1)}$ and $C_{2}^{(k+1)}$ we write the last renormalization condition in (H.5) in the form

$$
\begin{aligned}
& v_{u}^{(k+1, \infty)}-\beta\left\{\left(a+\beta^{-1} c\right) \zeta \frac{a}{(a+v)^{2}}\left[1+\frac{v}{12}\left(1-L^{-2} \eta^{2}\right)-v^{2} c_{1}(v)\right]+\beta^{-1} c \frac{a v}{(a+v)^{3}}\right. \\
& \quad \times\left[1+\frac{v}{12}\left(1-L^{-2} \eta^{2}\right)-v^{2} c_{1}(v)\right]+\left(\frac{a+\beta^{-1} c}{1+\frac{a+v}{\lambda}} \zeta+v \beta^{-1} e\right) \frac{a^{2}}{(a+v)^{3}} \\
& \quad \times\left[-1+\frac{a-v}{24}\left(1-L^{-2} \eta^{2}\right)-a v c_{1}(v)+\frac{1}{2}(a+v) v^{2} c_{2}(v)\right] \\
& \left.+\beta^{-1} b \frac{1}{2} \frac{a^{2}}{(a+v)^{2}}\left[1+v^{2} c_{1}(v)-v^{3} c_{2}(v)\right]\right\}+\beta v^{(\infty)}\left(C_{2}^{(k+1)}\right)=0 .
\end{aligned}
$$

This is a third equation for the renormalization constants, so we have the three Eqs. (5.5), (5.14) for five unknown constants. To analyze these equations we have to obtain bounds for various functions occurring in them. 
Let us start with the first term in (5.14). We obtain from (H.5)

$$
v_{u}^{(k+1, \infty)}=-\frac{1}{2} \sum_{Y \in \mathscr{D}_{k+1}\left(L^{-1} \eta \mathbb{Z}^{d}\right): 0 \in Y} \sum_{x, z \in Y}\left(\frac{\partial^{2}}{\partial \psi_{2}(x) \partial \psi_{2}(0)} \mathscr{E}_{u}^{(k+1)}\right)\left(z, Y ; e_{1}, e_{1}\right) x_{1}^{2} .
$$

By Lemma $3.2[1]$ the functions $\mathscr{E}_{u}^{(k+1)}\left(z, Y ; e_{1}+\zeta e_{2}, e_{1}\right)$ are analytic on the complex domain

$\left\{\zeta: \zeta\right.$ is a $\mathbb{C}$-valued function defined on $\left.T_{1}^{(k+1)},|\zeta|<\varepsilon_{0}+\delta_{0},\left|\partial^{1} \zeta\right|<\delta_{0},|\zeta|^{2}<\delta_{0}\right\}$ if $\varepsilon_{0}+K_{1} \delta_{0} \leqq \varepsilon_{k+1}$, in particular for $\varepsilon_{0}=\delta_{0}=\frac{1}{2} K_{1}^{-1} \varepsilon_{k+1}$.

This domain contains the domain $\left\{\zeta:|\zeta|<\frac{1}{2} \delta_{0}\right\}$, hence we obtain the estimate

$$
\left|\left\langle\left(\frac{\partial^{2}}{\partial \psi_{2} \partial \psi_{2}} \mathscr{E}_{u}^{(k+1)}\right)\left(z, Y ; e_{1}, e_{1}\right), \delta \zeta_{1}, \delta \zeta_{2}\right\rangle\right| \leqq\left(\frac{4}{\delta_{0}}\right)^{2} B_{6} \exp \left(-\kappa d_{k+1}(Y)\right)\left|\delta \zeta_{1}\right|\left|\delta \zeta_{2}\right|
$$

for arbitrary functions $\delta \zeta_{1}, \delta \zeta_{2}$. We write $x_{1}^{2}=\left(x_{1}-z_{1}\right)^{2}+2\left(x_{1}-z_{1}\right) z_{1}+z_{1}^{2}$ and take $\delta \zeta_{1}$ as one of the functions $\left(x_{1}-z_{1}\right)^{2}, x_{1}-z_{1}, 1$, and $\delta \zeta_{2}$ as a corresponding function $\delta, z_{1} \delta, z_{1}^{2} \delta$, where $\delta$ is the unit lattice $\delta$-function concentrated at 0 . The sum over $x$ in $(5.15)$ can be written as a sum of three terms corresponding to the three choices of the functions $\delta \zeta_{1}, \delta \zeta_{2}$, and using (5.16) it can be bounded by

$$
4\left(\frac{4}{\delta_{0}}\right)^{2} B_{6} \exp \left(-\kappa d_{k+1}(Y)\right) \sup _{x \in Y}|x-z|^{2} .
$$

We have $|x-z| \leqq\left|\Gamma_{x, z}\right| \leqq 2 d L M+d M d_{k+1}(Y)$ by (3.54)[1]. The sum over $z \in Y$ in (6.15) gives the factor $|Y|$. Using the inequality $|Y| \leqq(3 L M)^{d}\left(1+d_{k+1}(Y)\right)$, which will be proved in the paper on localization expansions, we obtain the bound

$$
\begin{aligned}
& 4\left(\frac{4}{\delta_{0}}\right)^{2} B_{6} \exp \left(-\kappa d_{k+1}(Y)\right)(2 d L M)^{2}\left(1+\frac{1}{2 L} d_{k+1}(Y)\right)^{2}(3 L M)^{d}\left(1+d_{k+1}(Y)\right) \\
& \quad<4\left(16 d K_{1} L M\right)^{2}(3 L M)^{d} B_{6} \frac{1}{\varepsilon_{k+1}^{2}} \exp \left(-(\kappa-2) d_{k+1}(Y)\right)
\end{aligned}
$$

The sum over $Y$ in (5.15) can be bounded by $K_{0}$ using the above exponential factors, so we obtain finally the bound

$$
\left|v_{u}^{(k+1, \infty)}\right|<2 K_{0}\left(16 d K_{1} L M\right)^{2}(3 L M)^{d} B_{6} \frac{1}{\varepsilon_{k+1}^{2}} .
$$

Let us recall that $v_{u}^{(k+1, \infty)}$ is an analytic function of the renormalization constants $\xi$, and the above bound holds on the polydisc $\left\{\xi_{:}\left|\xi_{i}\right|<c_{10}\right\}$. From this we can obtain bounds on derivatives of $v_{u}^{(k+1, \infty)}$ with respect to $\xi$.

Consider the last term in (5.14). The constant $v^{(\infty)}\left(C_{2}^{(k+1)}\right)$ is given by the formula (5.15) with $C_{2}^{(k+1)}$ instead of $\mathscr{E}_{u}^{(k+1)}$. The function $C_{2}^{(k+1)}(z)$ has been analyzed in Proposition 4.1. In the proof of (5.17) we have used only the 
general properties established there, so we obtain the following bound also:

$$
\left|v^{(\infty)}\left(C_{2}^{(k+1)}\right)\right|<2 K_{0}\left(16 d K_{1} L M\right)^{2}(3 L M)^{d} C_{4} \varepsilon^{2} .
$$

The function $v^{(\infty)}\left(C_{2}^{(k+1)}\right)$ is an analytic function of at least second order on the polydisc (4.8). Let us remark that a much better bound can be proved for this function, without the factor $(L M)^{d+2}$, if we use better analyticity properties of $C_{2}^{(k+1)}$, or by more explicit representations, as in the case of $C_{1}^{(k+1)}$, but it is not important here. The above statements about $C_{2}^{(k+1)}(z)$ apply also to the case of constant spin configurations, i.e., to the function $c_{2}^{(k+1)}(u, v, 0)$. It is an analytic function of $u, v$ on the discs described after (5.1), and of $\xi$ on the polydisc (4.8), and it is bounded by $C_{4} \varepsilon_{k+1}^{2} \varepsilon^{2}$ on those domains. Thus the derivatives satisfy the bounds

$$
\begin{gathered}
\left|\left(\frac{\partial}{\partial u} c_{2}^{(k+1)}\right)(1,1,0)\right|<C_{4} \varepsilon_{k+1}^{2} \varepsilon^{2} \frac{3}{\delta_{0}}=6 C_{4} K_{1} \varepsilon_{k+1} \varepsilon^{2}, \\
\left|\left(\frac{\partial}{\partial v} c_{2}^{(k+1)}\right)(1,1,0)\right|<C_{4} \varepsilon_{k+1}^{2} \varepsilon^{2} \frac{v}{\delta_{0}^{2}}=4 C_{4} K_{1}^{2} v \varepsilon^{2} .
\end{gathered}
$$

Consider now the system of Eqs. (5.5),(5.14). We have three equations for five unknown constants, so we have to supplement them with two additional equations. Obviously most important terms in the system are linear terms in $\xi$. Besides linear terms written explicitly there are linear terms coming from expansions of the functions $\left(\frac{\partial}{\partial u} f_{u}^{(k+1)}\right)(1,1,0),\left(\frac{\partial}{\partial v} f_{u}^{(k+1)}\right)(1,1,0), v_{u}^{(k+1, \infty)}$ in $\xi$, but these are very small in comparison with the first, which are multiplied by $\beta$, so we have to consider only the explicitly written linear terms. A closer inspection of these terms shows that the constant $\beta^{-1} d$ does not appear at all, and $\beta^{-1} c$ is multiplied by a coefficient proportional to $v$, which is small for a large number of steps. Thus the equations are singular in $\beta^{-1} c, \beta^{-1} d$ and these constants must be determined by some independent conditions. Intuitively a reason for this is clear, the corresponding expressions multiplied by these constants in the effective action $A_{k+1, u}$, or in $C^{(k+1)}$, are irrelevant, so they make unessential contributions only. There is no need for their renormalization, so we may choose $\beta^{-1} c, \beta^{-1} d$ basically in an arbitrary way. One obvious choice is to take $c=0, d=0$. There are plenty of other possible choices, and we discuss here one of them, obtained by requiring that the constants $a_{k}$ are determined by a "free" renormalization group flow, i.e. by the recursive equations $a_{k+1}=\frac{a_{k} a}{a_{k}+a L^{-2}}, a_{1}=a$, and the constants $\lambda_{k}$ are determined by "pure scaling", i.e. by the equations $\lambda_{k+1}=\lambda_{k} L^{2}$. The constants $a_{k+1, u}, \lambda_{k+1, u}$ are obtained by such equations from $a_{k}, \lambda_{k}$, so these requirements can be written as $a_{k+1}=a_{k+1, u}$, $\lambda_{k+1}=\lambda_{k+1, u}$. On the other hand by the renormalization group equations (4.1) we have

$$
\begin{array}{r}
\beta_{k+1, u} a_{k+1, u}=\left(\beta_{k+1}+b_{k+1}\right) a_{k+1}=\beta_{k+1} a_{k+1}+c_{k+1}, \quad \text { hence } c_{k+1}=a_{k+1} b_{k+1}, \\
\frac{\beta_{k+1, u}}{\lambda_{k+1, u}}=\frac{\beta_{k+1}+b_{k+1}}{\lambda_{k+1}}=\frac{\beta_{k+1}}{\lambda_{k+1}}+\frac{1}{\lambda_{k+1}} d_{k+1}, \quad \text { hence } d_{k+1}=b_{k+1}
\end{array}
$$

In the simplified notation the requirements lead to the equations $c=a b, d=b$, which yield an example of the additional conditions on the renormalization constants. 
We consider the Eqs. (5.5),(5.14) with these substitutions, so we obtain a system of three equations for the three unknown constants $\zeta, \beta^{-1} e, \beta^{-1} b$. We analyze now these equations. Let us remark that this analysis applies also to other "reasonable" choices of $c, d$, possibly with some unimportant changes of constants in bounds. We divide the equations by $\beta$, and we divide also the first equation in (5.5) by $\varepsilon_{k+1}$. The obtained system of equations is

$$
\begin{aligned}
& a_{11} \frac{\zeta}{\varepsilon_{k+1}}+\left[a_{11} \frac{\zeta}{\varepsilon_{k+1}} \beta^{-1} b+\varepsilon_{k+1} a\left(1+\beta^{-1} b\right)\left(\frac{\zeta}{\varepsilon_{k+1}}\right)^{2}-\frac{1}{\varepsilon_{k+1}}\left(\frac{\partial}{\partial u} c_{2}^{(k+1)}\right)(1,1,0)\right] \\
& \quad=\beta^{-1} \frac{1}{\varepsilon_{k+1}}\left(\frac{\partial}{\partial u} f_{u}^{(k+1)}\right)(1,1,0) \\
& \quad a_{21} \frac{\zeta}{\varepsilon_{k+1}}+a_{22} \beta^{-1} e+\left[a_{21} \frac{\zeta}{\varepsilon_{k+1}} \beta^{-1} b+\frac{1}{v}\left(\frac{\partial}{\partial v} c_{2}^{(k+1)}\right)(1,1,0)\right] \\
& \quad=-\beta^{-1} \frac{1}{v}\left(\frac{\partial}{\partial v} f_{u}^{(k+1)}\right)(1,1,0), \\
& \quad a_{31} \frac{\zeta}{\varepsilon_{k+1}}+a_{32} \beta^{-1} e+a_{33} \beta^{-1} b+\left[a_{31} \frac{\zeta}{\varepsilon_{k+1}} \beta^{-1} b-v^{(\infty)}\left(C_{2}^{(k+1)}\right)\right]=\beta^{-1} v_{u}^{(k+1, \infty)},
\end{aligned}
$$

where

$$
\begin{aligned}
a_{11}= & a \frac{\lambda+v}{\lambda+a+v}, \quad a_{21}=\varepsilon_{k+1} \frac{a}{\lambda+a+v}, \quad a_{22}=1, \\
a_{31}= & \varepsilon_{k+1} \frac{a^{2}}{(a+v)^{2}}\left[1+\frac{v}{12}\left(1-L^{-2} \eta^{2}\right)-v^{2} c_{1}(v)\right] \\
& +\varepsilon_{k+1} \frac{a^{3}}{(a+v)^{3}}\left[-1+\frac{a-v}{24}\left(1-L^{-2} \eta^{2}\right)-a v c_{1}(v)+\frac{1}{2}(a+v) v^{2} c_{2}(v)\right], \\
a_{32}= & \frac{a^{2} v}{(a+v)^{3}}\left[-1+\frac{a-v}{24}\left(1-L^{-2} \eta^{2}\right)-a v c_{1}(v)+\frac{1}{2}(a+v) v^{2} c_{2}(v)\right], \\
a_{33}= & \frac{1}{2} \frac{a^{2}}{(a+v)^{2}}\left[1+v^{2} c_{1}(v)-v^{3} c_{2}(v)\right]+\frac{a^{2} v}{(a+v)^{3}}\left[1+\frac{v}{12}\left(1-L^{-2} \eta^{2}\right)-v^{2} c_{1}(v)\right] .
\end{aligned}
$$

The explicitly written linear part of the system is given by the triangular matrix with the above coefficients, so it is important to get bounds for the coefficients $a_{11}, a_{22}, a_{33}$ on the main diagonal. To get slightly better bounds we assume that $1<a<\frac{3}{2}$, i.e. all $a_{k}$ are in the interval $] 1, \frac{3}{2}[$. They are given by the "free flow" formula $a_{k}=a \frac{1-L^{-2}}{1-L^{-2 k}}$, so it is enough to take $a \in\left[\left(1-L^{-2}\right)^{-1}, \frac{3}{2}[\right.$, e.g. an optimal choice is $a=\left(1-L^{-2}\right)^{-1}$, and then $a_{k} \searrow 1$. With these assumptions we can easily prove the following bounds:

$$
\begin{gathered}
\frac{1}{2}<a_{11}<\frac{3}{2}, \quad a_{22}=1, \quad \frac{1}{4}<a_{33}<1, \quad \text { and } \quad\left|a_{21}\right|<\varepsilon_{k+1}, \\
\left|a_{31}\right|<\frac{13}{6} \varepsilon_{k+1}<\frac{5}{2} \varepsilon_{k+1}, \quad\left|a_{32}\right|<\frac{14}{13} v<\frac{3}{2} .
\end{gathered}
$$


Thus the matrix is regular and its inverse can be calculated by a simple Gauss elimination procedure. Applying it to the system (5.21), and using the notations (5.2), we obtain a system of equations of the following form:

$$
\xi+c(\xi)=f(\xi)
$$

where $\xi$ is now a three-component vector with components $\xi_{1}=\frac{\zeta}{\varepsilon_{k+1}}, \quad \xi_{2}=\beta^{-1} e$, $\xi_{3}=\beta^{-1} b$. The functions $c_{i}(\xi), i=1,2,3$, are suitable linear combinations of the functions in square brackets in (5.21), so they are analytic functions of at least second order in $\xi$. From the Gauss elimination procedure and the bounds (5.17), (5.18) we obtain the bounds

$$
\left|c_{i}(\xi)\right|<9 K_{0}\left(16 d K_{1} L M\right)^{2}(3 L M)^{d} C_{4} \varepsilon^{2}, \quad i=1,2,3,
$$

for $\xi$ in the polydisc $\left\{\xi:\left|\xi_{i}\right|<c_{10}\right\}$. We have written above a common bound for all the functions. The functions $f_{i}(\xi)$ are the same linear combinations of the functions on the right-hand sides of (5.21), so they are analytic functions of $\xi$ defined on the polydisc and satisfying the bounds

$$
\left|f_{i}(\xi)\right|<9 K_{0}\left(16 d K_{1} L M\right)^{2}(3 L M)^{d} B_{6} \beta^{-1} \frac{1}{\varepsilon_{k+1}^{2}} .
$$

Notice that the number on the right-hand side above is arbitrarily small if $\beta$ is large enough. Consider the system (5.23). The right-hand side can be arbitrarily small, and the function $c(\xi)$ is of second order in $\xi$, so the system should have exactly one small solution. To prove it we notice that a solution is a fixed point of the mapping $\xi \rightarrow-c(\xi)+f(\xi)$, so it is enough to prove that the mapping is a contraction on some polydisc $\left\{\xi:|\xi|_{\infty}<\varepsilon\right\}$, where $|\xi|_{\infty}=\max _{i}\left|\xi_{i}\right|$ and $\varepsilon \leqq c_{10}$. For simplicity denote $K_{5}=9 K_{0}\left(16 d K_{1} L M\right)^{2}(3 L M)^{d}$. We have the following bounds for derivatives of $c_{i}(\xi), f_{i}(\xi)$ on the polydisc with $\varepsilon \leqq \frac{1}{2} c_{10}$ :

$$
\sum_{j=1}^{3}\left|\frac{\partial}{\partial \xi_{j}} c_{i}(\xi)\right|<4 C_{4} K_{5} \varepsilon, \quad \sum_{j=1}^{3}\left|\frac{\partial}{\partial \xi_{j}} f_{i}(\xi)\right|<4 B_{6} K_{5} \frac{1}{c_{10}} \beta^{-1} \frac{1}{\varepsilon_{k+1}^{2}} .
$$

They imply that

$$
\begin{aligned}
& \left|\left[-c\left(\xi^{\prime}\right)+f\left(\xi^{\prime}\right)\right]-\left[-c\left(\xi^{\prime \prime}\right)+f\left(\xi^{\prime \prime}\right)\right]\right|_{\infty} \\
& \leqq\left(4 C_{4} K_{5} \varepsilon+4 B_{6} K_{5} \frac{1}{c_{10}} \beta^{-1} \frac{1}{\varepsilon_{k+1}^{2}}\right)\left|\xi^{\prime}-\xi^{\prime \prime}\right|_{\infty} \leqq \frac{1}{2}\left|\xi^{\prime}-\xi^{\prime \prime}\right|_{\infty} \\
& \quad \text { if } 16 C_{4} K_{5} \varepsilon \leqq 1,16 B_{6} K_{5} \frac{1}{c_{10}} \beta^{-1} \frac{1}{\varepsilon_{k+1}^{2}} \leqq 1
\end{aligned}
$$

The first condition gives a restriction on the radius of the polydisc, it determines the maximal radius $r_{0}=\min \left\{\left(16 C_{4} K_{5}\right)^{-1}, \frac{1}{2} c_{10}\right\}$. The second condition gives a restriction on $\beta$. Assuming these conditions we obtain that the mapping is a contraction, hence Eq. (5.23) has at most one solution. A solution can be constructed as a limit of a sequence of successive approximations, starting for example with $f(0)$, if $|f(0)|_{\infty}<\frac{1}{2} r_{0}$. Then the sequence is convergent and all elements of the sequence can be bounded by $2|f(0)|_{\infty}$. The limit satisfies this inequality also, and it is a solution of (5.23). Consider the condition $|f(0)|_{\infty}<\frac{1}{2} r_{0}$. By (5.25) it is 
enough to assume that $2 B_{6} K_{5} \beta^{-1} \frac{1}{\varepsilon_{k+1}^{2}} \leqq r_{0}$. By the definition of $r_{0}$ this is equivalent to two conditions. We write them together with the second condition (5.26) in the form

$$
16 B_{6} K_{5} \max \left\{2 C_{4} K_{5}, \frac{1}{c_{10}}\right\} \beta_{k+1}^{-1} \frac{1}{\varepsilon_{k+1}^{2}} \leqq 1,
$$

where we have written explicitly that $\beta$ equals $\beta_{k+1}$. If this condition is satisfied, then all the above statements and bounds hold, in particular Eq. (5.23) have a unique solution $\xi^{0}$ satisfying the bound $\left|\xi^{0}\right|_{\infty} \leqq 2|f(0)|_{\infty}<2 B_{6} K_{5} \beta_{k+1}^{-1} \frac{1}{\varepsilon_{k+1}^{2}}$. Let us analyze this condition. From Eqs. (4.1) and Definition (5.2) it is easy to calculate that

$$
\beta_{k+1}=\beta_{k+1, u} \frac{1}{1+\xi_{3}}=\beta_{k} L^{d-2} \frac{1}{1+\xi_{3}}, \quad v_{k+1}=v_{k+1, u} \frac{1+\xi_{3}}{1+\xi_{2}}=v_{k} L^{2} \frac{1+\xi_{3}}{1+\xi_{2}} .
$$

From the first equality and the inductive hypothesis (H.6) we get

$$
\begin{gathered}
\beta_{k+1}^{-1}<\frac{3}{2} \beta^{-1} L^{-(k+1)(d-2)} \gamma_{k}<2 \beta^{-1} L^{-(k+1)(d-2)}, \text { hence } \\
\beta_{k+1}^{-1} \frac{1}{\varepsilon_{k+1}^{2}}<\frac{2}{\alpha_{0}^{2}} \beta^{-1} L^{-(k+1) 2 \gamma}<\frac{2}{\alpha_{0}^{2}} \beta^{-1},
\end{gathered}
$$

and condition (5.27) is satisfied if

$$
32 B_{6} K_{5} \max \left\{2 C_{4} K_{5}, \frac{1}{c_{10}}\right\} \frac{1}{\alpha_{0}^{2}} \beta^{-1} \leqq 1 .
$$

Assuming this we obtain that all the previous conditions are satisfied, so this completes the analysis of Eqs. (5.5),(5.14). For the solution $\xi^{0}$ of these equations we have

$$
\left|\xi^{0}\right|_{\infty}<2 B_{6} K_{5} \beta_{k+1}^{-1} \frac{1}{\varepsilon_{k+1}^{2}}<4 B_{6} K_{5} \frac{1}{\alpha_{0}^{2}} \beta^{-1} L^{-2 \gamma(k+1)}<\frac{1}{4} c_{8} L^{-2 \gamma(k+1)},
$$

if $\beta$ is large enough. The equalities (5.28) and the inductive hypothesis (H.6) imply

$$
\begin{aligned}
& \gamma_{k+1}=\gamma_{k} \frac{1}{1+\xi_{3}^{0}}, \quad \delta_{k+1}=\delta_{k} \frac{1+\xi_{3}^{0}}{1+\xi_{2}^{0}}, \\
& \gamma_{k+1}, \gamma_{k+1}^{-1}, \delta_{k+1}, \delta_{k+1}^{-1}<\exp \left(c_{8} \sum_{j=1}^{k} L^{-2 \gamma j}\right) \\
& \quad \times \max \left\{\frac{1}{1+\xi_{3}^{0}}, 1+\xi_{3}^{0}, \frac{1+\xi_{3}^{0}}{1+\xi_{2}^{0}}, \frac{1+\xi_{2}^{0}}{1+\xi_{3}^{0}}\right\} \leqq \exp \left(c_{8} \sum_{j=1}^{k} L^{-2 \gamma j}\right) \\
& \quad \times \exp \left(\left|\log \left(1+\xi_{3}^{0}\right)\right|+\left|\log \left(1+\xi_{2}^{0}\right)\right|\right)<\exp \left(c_{8} \sum_{j=1}^{k+1} L^{-2 \gamma j}\right) .
\end{aligned}
$$


This proves the inductive hypothesis for the renormalized constants $\beta_{k+1}, a_{k+1}$, $\lambda_{k+1}, v_{k+1}$.

Let us make two remarks on the above analysis. The first is that a dependence on $\xi$ in Eqs. (5.5), (5.14), or (5.23), is somewhat more subtle, besides the explicitly written one there is also a dependence through the constants $\beta_{k+1}, v_{k+1}$ given by (5.28), but this does not change anything in the considerations above. The second is that the equalities (5.32) and the bound (5.31) lead to the following precise form of the asymptotic behavior of the "running" coefficients $\beta_{k}, v_{k}$ :

$$
\lim _{k \rightarrow \infty} \frac{\beta_{k}}{\beta L^{k(d-2)}}=\lim _{k \rightarrow \infty} \gamma_{k}=\gamma_{\infty}, \quad \lim _{k \rightarrow \infty} \frac{v_{k}}{v L^{2 k}}=\lim _{k \rightarrow \infty} \delta_{k}=\delta_{\infty},
$$

and $\gamma_{\infty}, \delta_{\infty}$ satisfy the inequalities (5.32) with $k+1$ replaced by $\infty$. Finally notice that if $\xi^{0}$ is given, then by (5.28) we have $\beta_{k+1}, v_{k+1}$, hence we can calculate the constants $b_{k+1}=\beta_{k+1} \xi_{3}^{0}, e_{k+1}=\beta_{k+1} \xi_{2}^{0}$. Thus we have proved the following proposition:

Proposition 5.1. The system of Eqs. (5.5),(5.14) supplemented with the equations $c=a b, d=b$, or equivalently the system (5.23), has exactly one solution in the polydisc $\left\{\xi \in \mathbb{C}^{3}:|\xi|_{\infty}<r_{0}\right\}$, where $\xi_{1}=\varepsilon_{k+1}^{-1} \zeta, \xi_{2}=\beta^{-1} e, \xi_{3}=\beta^{-1} b$, if the condition (5.30) is satisfied. The solution $\xi^{0}$ is real and satisfies the bound $\left|\xi^{0}\right|_{\infty} \leqq 2|f(0)|_{\infty}<2 B_{6} K_{5} \beta_{k+1}^{-1} \frac{1}{\varepsilon_{k+1}^{2}}$, hence the bound (5.31) and the above one implies the bounds (5.32) for $\gamma_{k+1}, \delta_{k+1}$ and the inductive hypothesis (H.6) for the "new" coefficients $\beta_{k+1}, a_{k+1}, \lambda_{k+1}, v_{k+1}$.

The above analysis was done assuming the additional equations $c=a b, d=b$, but it holds also for the second natural choice of the equations, namely $c=0, d=0$. In fact all the above bounds and results hold for this choice without any changes, except that the equalities (5.28) are complemented with the equalities

$$
a_{k+1}=\frac{a a_{k}}{a_{k}+a L^{-2}}\left(1+\xi_{3}\right), \quad \lambda_{k+1}=\lambda_{k} L^{2} \frac{1}{1+\xi_{3}} .
$$

They yield the second set of equalities in (H.6). We could also consider some mixed sets of equations between the two above sets, for example $c=0, d=b$, and the results would still hold for such choices.

The construction of the solution of Eqs. (5.5), (5.14) completes the definition of the renormalization transformation. We substitute the solution into the function $\mathscr{E}^{(k+1)}$ defined by (4.22), and the obtained function satisfies the renormalization conditions (H.5) also. We need only to make a few final comments on bounds for this function, or rather for terms of its localization expansion. We define

$$
\mathscr{E}^{(k+1)}(z, Y)=\mathscr{E}_{u}^{(k+1)}(z, Y)-\beta_{k+1} C^{(k+1)}(z, Y), \quad Y \in \mathscr{D}_{k+1}, z \in Y,
$$

and if $Y$ is a cube from $\pi_{k+1}^{\prime}$ containing $z$ in its central large cube, then we also subtract $E^{(k+1)}(z)$. From Proposition 4.1 and (5.31) we obtain

$$
\begin{aligned}
\left|\mathscr{E}^{(k+1)}(z, Y)\right| & <\left(B_{6}+2 C_{4} B_{6} K_{5}+4 C_{4} B_{6}^{2} K_{5}^{2} \beta_{k+1}^{-1} \frac{1}{\varepsilon_{k+1}^{2}}\right) \exp \left(-\kappa d_{k+1}(Y)\right) \\
& <\left(B_{6}+2 C_{4} B_{6} K_{5}+\frac{1}{2} C_{4} B_{6} K_{5} c_{8}\right) \exp \left(-\kappa d_{k+1}(Y)\right) \\
& <3 C_{4} K_{6} K_{5} \exp \left(-\kappa d_{k+1}(Y)\right)
\end{aligned}
$$


for domains $Y$ different from the above cube. For the constant $E^{(k+1)}(z)$ we obtain therefore the bound

$$
\left|E^{(k+1)}(z)\right|<3 C_{4} B_{6} K_{0} K_{5},
$$

and the final bounds for terms of the localization expansion of $\mathscr{E}^{(k+1)}(z)$ are

$$
\left|\mathscr{E}^{(k+1)}(z, Y)\right|<4 C_{4} B_{6} K_{0} K_{5} \exp \left(-\kappa d_{k+1}(Y)\right)
$$

for all localization domains $Y \in \mathscr{D}_{k+1}, Y \ni z$ (assuming $K_{0} \geqq 3$ ). The constants in the above inequality are independent of $E_{0}$, so we have to assume that

$$
E_{0} \geqq 4 C_{4} B_{6} K_{0} K_{5}
$$

for example we can define it by the equality above. This completes the analysis of the new contribution to the effective action.

Consider now the new contribution to the generating functional. We have proved all the properties in the inductive hypothesis (H.7), except the bounds for terms of the localization expansions. In the bounds formulated in Proposition 4.2 we substitute $\varepsilon=4 B_{6} K_{5} \frac{1}{\alpha_{0}^{2}} \beta^{-1} L^{-2 \gamma(k+1)}$, on the basis of the inequalities (5.31). The constant there is equal to

$$
\frac{1}{2} \beta^{-\frac{1}{4}}+4 B_{6} C_{6} K_{5} \frac{1}{\alpha_{0}^{2}} \beta^{-1} L^{-\alpha_{1}(k+1)}<\beta^{-\frac{1}{4}} \leqq c_{9}
$$

for $\beta$ large enough so we obtain the bounds in the inductive hypothesis. We can define $c_{9}=\beta^{-\frac{1}{4}}$.

Let us come back yet to the condition (4.24), which is $C_{5} E_{0} \varepsilon \leqq 1$, and take $\varepsilon=4 B_{6} K_{5} \frac{1}{\alpha_{0}^{2}} \beta^{-1}$ by (5.31). The condition can be written as

$$
4 B_{6} C_{5} K_{5} \frac{1}{\alpha_{0}^{2}} E_{0} \beta^{-1} \leqq 1, \text { or even } 16 B_{6}^{2} C_{4} C_{5} K_{0} K_{5}^{2} \frac{1}{\alpha_{0}^{2}} \beta^{-1} \leqq 1 \text {, }
$$

if we take $E_{0}$ given by the equality in (5.39), i.e., $E_{0}=4 C_{4} B_{6} K_{0} K_{5}$. It is the strongest restriction on $\beta$. It could be essentially improved by a more careful, but much lengthier, analysis than the one given here.

Now we have completed the analysis of the renormalization operation. We can formulate the obtained results in the proposition.

Proposition 5.2. Substituting the solution of the system of Eqs. (5.5), (5.14) into the functions $\mathscr{E}^{(k+1)}, \mathscr{F}^{(k+1)}(g)$ defined by formulas $(4.20)-(4.22),(4.18)$, we obtain the final $k+1^{\text {st }}$ contributions to the effective action and the generating functional They satisfy the inductive hypotheses (H.2)-(H.5),(H.7) correspondingly, if $\beta$ is large enough, and $E_{0}$ satisfies the restriction (5.39).

This completes also the proof of Theorem 2. To complete the proof of Theorem 1 we have to multiply the density (5.21) by the characteristic function $\chi_{k+1}$, and to notice the identity $\chi_{k+1} \chi_{k+1, u}=\chi_{k+1}$. We could write now the constants and the restrictions in the formulations of these theorems more explicitly, but it is not important. 


\section{The Last Step Procedure}

We have stated already that we finish the renormalization procedure if the external field $v_{k}$ is close to $1, v_{k} \approx 1$. We have to formulate this statement precisely now. Ideally we would like to have $v_{k}=1$, but if we start with an arbitrary $v$ we almost never get this value. We assume that the renormalization steps are finished when $v_{k}$ is for the first time greater than a fixed value, e.g. $v_{k}>\frac{7}{8} L^{-2}$. The sequence $v_{k}$ is strictly increasing, so the index $k$ is uniquely defined. By the definition $v_{k-1} \leqq \frac{7}{8} L^{-2}$, hence $v_{k, u}=v_{k-1} L^{2} \leqq \frac{7}{8}$ and $v_{k}<1$. Denote the index $k$ by $k_{1}$, it is a function of $v$, and of course of the other parameters also, but the dependence on $v$ is most important. Consider the density $\rho_{k_{1}}$, and in particular the characteristic function $\chi_{k_{1}}$. The domain of this function is equal to the space $\Xi_{k_{1}}\left(1, \beta_{k_{1}}^{-\frac{1}{2}} p\left(\beta_{k_{1}}\right)\right)$, and by Lemma 3.1 and (3.21)[1] we have

$$
\tilde{\Psi}_{k_{1}}\left(K_{1}^{-1} \delta_{k_{1}}\right) \subset \Xi_{k_{1}}\left(1, \delta_{k_{1}}\right) \subset \tilde{\Psi}_{k_{1}}\left(3 \delta_{k_{1}}\right), \text { where } \delta_{k_{1}}=\beta_{k_{1}}^{-\frac{1}{2}} p\left(\beta_{k_{1}}\right) .
$$

This means that the restrictions defining the space are basically equivalent to the restrictions defining $\tilde{\Psi}_{k_{1}}\left(\delta_{k_{1}}\right)$. In this step we take finally vectors $h$ in the unit sphere, $h \in S^{N-1}$. Then the third condition in the definition (3.18)[1] of the space $\tilde{\Psi}_{k_{1}}\left(3 \delta_{k_{1}}\right)$ can be written as $\frac{1}{2} v_{k_{1}}\left|\left(\psi_{k_{1}}\right)_{0}-h\right|^{2}<9 \delta_{k_{1}}^{2}$. Taking into account the definition of $k_{1}$ and the second condition there we obtain that

$$
\left|\psi_{k_{1}}-h\right|<(5 L+3) \delta_{k_{1}}
$$

on the domain of the characteristic function $\chi_{k_{1}}$. The above restriction of $\psi_{k_{1}}$ to a neighborhood of the vector $h$ is of the same order as the other restrictions on $\psi_{k_{1}}$. This is a crucial observation, which allows us to perform the final integration with respect to the variables $\psi_{k_{1}}$ without any further renormalization transformations. Thus we consider the integral

$$
\int d \psi_{k_{1}} \chi^{\left(k_{1}\right)} \chi_{k_{1}} \exp \left[-\beta_{k_{1}} A_{k_{1}}\left(\psi_{k_{1}}, \phi_{k_{1}} ; h\right)+\mathscr{E}_{k_{1}}\left(\psi_{k_{1}}, h\right)-E_{k_{1}}\left|T_{1}^{\left(k_{1}\right)}\right|+\mathscr{F}_{k_{1}}\left(\psi_{k_{1}}, h, g\right)\right],
$$

where the fluctuation field characteristic function $\chi^{\left(k_{1}\right)}$ will be defined in a moment. We proceed in exactly the same way as in the previous steps. At first we look for critical points of the function $A_{k_{1}}\left(\psi_{k_{1}}, \phi_{k_{1}}, h\right)$. On the considered domain it has exactly one critical point, which is a minimum of this function. It is the constant configuration $\psi_{k_{1}}=h$, and then $\phi_{k_{1}}(h, h)=h$ also. We introduce the fluctuation field $\psi^{\prime}$ by the equality $\psi_{k_{1}}=h+\psi^{\prime}$, and we expand the expression in the exponential around the minimal configuration. In particular for the main action we have by $(2.26)$,

$$
A_{k_{1}}\left(\psi_{k_{1}}, \phi_{k_{1}} ; h\right)=\frac{1}{2}\left\langle\psi^{\prime}, \Delta^{\left(k_{1}\right)} \psi^{\prime}\right\rangle+V^{\left(k_{1}\right)}\left(\psi^{\prime}\right)
$$

because $A_{k}(h, h ; h)=0$. The quadratic form and the interaction are given by the formulas (2.23), (2.27), where $\phi_{k_{1}+1}=h$ and $\alpha_{k_{1}+1}=0$. The quadratic form satisfies inequality (2.24), and by the discussion after this inequality we have

$$
\frac{1}{2}\left\langle\psi^{\prime}, \Delta^{\left(k_{1}\right)} \psi^{\prime}\right\rangle \geqq \frac{1}{2} \gamma_{0}\left\langle\psi^{\prime},\left(-\Delta+v_{k_{1}}\right) \psi^{\prime}\right\rangle \geqq \frac{1}{2} \gamma_{0}\left\langle\psi^{\prime},\left(-\Delta+\frac{7}{8} L^{-2}\right) \psi^{\prime}\right\rangle .
$$


Thus the operator $\Delta^{\left(k_{1}\right)}$ is positive definite, e.g. $\Delta^{\left(k_{1}\right)} \geqq \frac{7}{8} \gamma_{0} L^{-2}$, and the lower bound is an absolute positive number. This is the basic fact which allows us to perform the fluctuation integral as in the previous steps, because the covariance $C^{\left(k_{1}\right)}$ is decaying exponentially with a decay rate of an order of the lower bound for $\Delta^{\left(k_{1}\right)}$. Notice that this lower bound is basically the same as lower bounds for the operators $\Delta^{(k)}+a Q^{*} Q$, which are determined by a lower bound of the operator $\gamma_{0}(-\Delta)$ $+a Q^{*} Q$, and this is approximately equal to $\gamma_{0} L^{-2}$. Having this basic property of $C^{\left(k_{1}\right)}$ we repeat the whole procedure described and discussed in the general $k+1^{\text {st }}$ step for the fluctuation integral, in particular we introduce the change of variables $\psi^{\prime}=\beta_{k_{1}}^{-\frac{1}{2}} C^{\left(k_{1}\right) \frac{1}{2}} \psi$, and the characteristic function $\chi\left(\left\{|\psi|<p_{1}\left(\beta_{k_{1}}\right)\right\}\right)$. We obtain an expression of the form

$$
\exp \left[\mathscr{E}_{k_{1}}(h, h)+\mathscr{E}_{0}^{\left(k_{1}+1\right)}(h, h)-E_{k_{1}}^{\prime \prime \prime}\left|T_{1}^{\left(k_{1}\right)}\right|+\mathscr{F}_{k_{1}}(h, h, g)+\mathscr{F}_{0}^{\left(k_{1}+1\right)}(h, h, g)\right] .
$$

By the first inductive assumption in (H.5) we have $\mathscr{E}_{k_{1}}(h, h)=0$, and we combine the remaining two constants to define $-E_{k_{1}+1}$. Actually we should define the constant $E$ in the original action in $(\mathrm{H} .1)$ as a normalization constant, which means that $E_{k_{1}+1}=0$. This determines uniquely $E$ in terms of all the contributions from all the steps. This is not the final definition of $E$ though, it has to be modified in the presence of large field contributions. The last term in the above exponential is equal to $\mathscr{F}^{\left(k_{1}+1\right)}$, so we obtain finally

$$
\exp \mathscr{F}_{k_{1}+1}(h, h, g)
$$

and the function in the exponential is the generating functional for connected correlation functions. There is no point in making an extensive analysis of this functional, this will be done in other papers, but let us point out several facts. The functional can be written as

$$
\mathscr{F}_{k_{1}+1}(h, h, g)=\langle g, h\rangle+\sum_{j=1}^{k_{1}+1}\left\langle g, \mathscr{M}^{(j)}(h, h, g)\right\rangle,
$$

and the terms satisfy the conditions (H.7), in particular the exponential tree decay property. They imply that we can take the thermodynamic limit $T \rightarrow \mathbb{Z}^{d}$, and for $v>0$ the correlation functions decay exponentially also. If $v \rightarrow 0$ then $k_{1} \rightarrow \infty$ by the asymptotic behavior (6.33) and there exists a limit of the generating functionals. This follows from the bounds in (H.7), which imply that the series above is uniformly convergent, and also from the fact that each term of the series has a limit as $v \rightarrow 0$. The limit $\mathscr{F}_{\infty}(h, h, g)$ is an analytic function of $g$, and it generates the connected correlation functions, which converge to 0 with separation of points going to $\infty$, so they define a pure thermodynamic phase.

\section{References}

1 Balaban, T: A low temperature expansion for classical $N$-vector models I A renormalization group flow Commun Math Phys. 167, 103-154 (1995)

2 Balaban, T: The variational problems for classical $N$-vector models Preprint, to appear in Commun Math Phys

3 Balaban, T: Commun Math. Phys 85, 603 (1982) 
4 Brydges, D, Dimock, J, Hurd, T : Weak perturbations of Gaussian measures Preprint

5 Cammarota, C: Commun Math Phys 85, 517-528 (1982)

6 Glimm, J, Jaffe, A : Quantum Physics A Functional Integral Point of View New York: Springer, 1989

Communicated by D Brydges 
